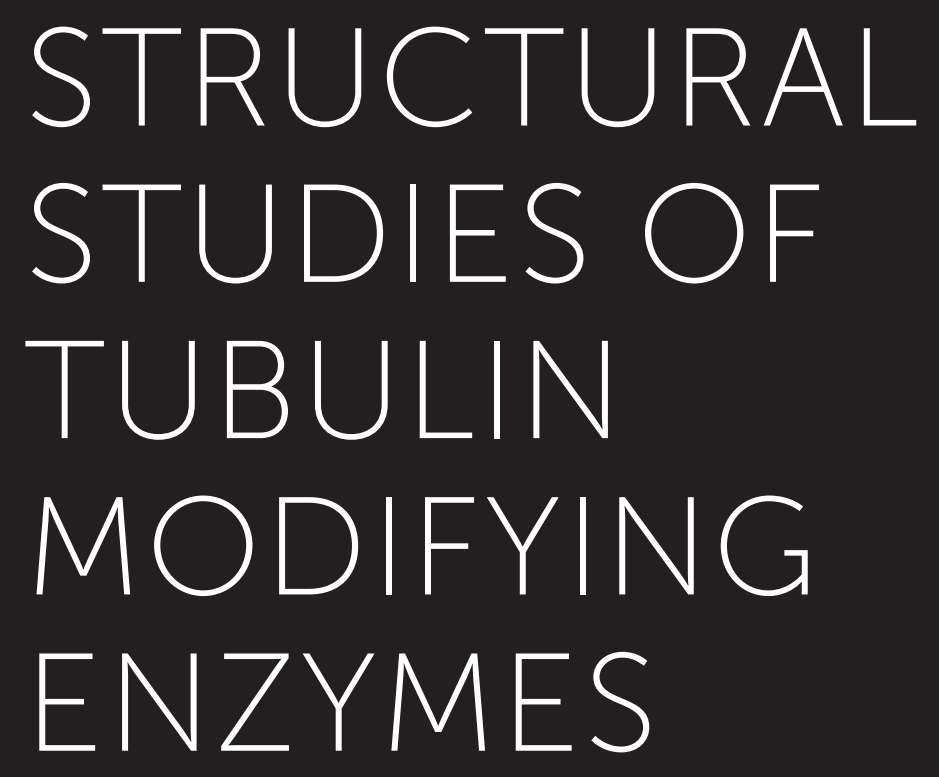

Athanasios Adamopoulos 



\section{STRUCTURAL STUDIES OF TUBULIN MODIFYING ENZYMES}

Athanasios Adamopoulos 


\section{COLOFON}

Structural studies of tubulin modifying enzymes PhD thesis, Utrecht University, The Netherlands Author: Nassos Adamopoulos ISBN/EAN: 978-94-6419-091-5

Copyright (C) 2020 Nassos Adamopoulos, Utrecht, The Netherlands.

All rights reserved. No parts of this thesis may be reproduced, stored in a retrieval system, or transmitted in any form or by any means without prior permission of the author. The copyright of articles that have been published has been transferred to the respective journals.

Layout and design: Ilse Modder https://www.ilsemodder.nl

Printing: Gildeprint | https://www.gildeprint.nl 


\title{
STRUCTURAL STUDIES OF TUBULIN MODIFYING ENZYMES
}

\section{Structureel onderzoek naar tubuline-modificerende enzymen}

(met een samenvatting in het Nederlands)

\author{
Proefschrift
}

\author{
ter verkrijging van de graad van doctor aan de \\ Universiteit Utrecht \\ op gezag van de \\ rector magnificus, prof.dr. H.R.B.M. Kummeling, \\ ingevolge het besluit van het college voor promoties \\ in het openbaar te verdedigen op \\ woensdag 3 februari 2021 des ochtends te 10.30 uur
}

door

\section{Athanasios Adamopoulos}

geboren op 17 februari 1987

te Marousi Athene, Griekenland 


\section{Promotoren:}

Prof. dr. A. Perrakis

Prof. dr. T.R. Brummelkamp 


\section{Leescommissie:}

Prof. A.S. Akhmanova

Prof. M. Baldus

Prof. A.M.J.J. Bonvin

Prof. P. Gros

Prof. T.K. Sixma

\section{Paranimphen:}

Tatjana Heidebrecht-Linnemann

Willem-Jan Keune 



\section{TABLE OF CONTENTS}

$\begin{array}{lll}\text { Chapter } 1 & \text { Introduction }\end{array}$

$\begin{array}{lll}\text { Chapter } 2 & \text { Vasohibins encode tubulin detyrosinating activity } & 29\end{array}$

Chapter $3 \quad$ Crystal structure of the VASH1/SVBP complex a tubulin tyrosine 59 carboxypeptidase

Chapter $4 \quad$ Mechanism and structure of MATCAP a new tyrosine metallo- 83 carboxypeptidase

$\begin{array}{lll}\text { Chapter } 5 & \text { General discussion } & 103\end{array}$

$\begin{array}{lll}\text { Addendum } & \text { Summary } & 112\end{array}$

Nederlandse samenvatting $\quad 114$

Curriculum Vitae 116

List of publications $\quad 117$

$\begin{array}{ll}\text { Acknowledgements } & 118\end{array}$ 



\title{
Chapter 1
}

\author{
Introduction
}


Eukaryotic cells are experiencing constantly a plethora of mechanical forces that are generated by their natural environment. These forces can arise from effects such as gravity, fluid flow, and active contraction generated not only by neighboring cells, but also by the extracellular matrix ${ }^{(1)}$. Cells are viscoelastic, which enables them to withstand mechanical forces which otherwise would cause deforming effects. Viscoelasticity is provided by the cytoskeleton, a three-dimensional network composed of protein filaments.

\section{THE CYTOSKELETON}

The cytoskeleton is comprised of three types of fibers: microfilaments, intermediate filaments and microtubules.

Microfilaments or actin filaments are the narrowest protein fibers of the cytoskeleton and are involved in cellular movement. They have a diameter of $7 \mathrm{~nm}$ and are assembled by two intertwined strands of the globular protein actin. Their dynamic nature allows them to grow and disassemble very fast, in a procedure controlled by adenosine triphosphate (ATP). They usually provide a trafficking network for protein molecules such as the molecular motor myosin, that moves along actin filaments. Their dynamic nature (rapid polymerization/de-polymerization) provides some rigidity to the cell, enabling them to change their shape and move.

Intermediate filaments are a cytoskeletal element composed of multiple strands of fibrous proteins bound together. They have an average diameter of 8-10 nm, between actin filaments and microtubules. They create supportive scaffolding inside the cell, and they can also bear tension. A number of proteins are found to form intermediate filaments (keratins, vimentin, desmin), making them the most diverse cytoskeletal element.

Microtubules are the last component of the eukaryotic cytoskeleton. They are hollow tubes with an average outer diameter of $25-27 \mathrm{~nm}$, whereas the inner diameter is $13-15 \mathrm{~nm}$. In cells the microtubule network is functionally specialized to assist in a number of cellular procedures such as motility, migration, cell division and intracellular transport. These filamentous components of the cytoskeleton do not only provide mechanical support, but also create a framework where a plethora of motor proteins localize using it as a track while moving. A few examples of these proteins are the dynein-dynactin complex, kinesins, and centromere protein E (CENP-E). These proteins move along the MT network through interactions with the cytoskeleton-associated protein glycine-rich (CAP-Gly) domains they contain and specific regions in the MT lattice. 


\section{THE MICROTUBULES}

Microtubules (MTs) are the largest non-covalent filamentous components of the eukaryotic cytoskeleton ${ }^{(2)}$. They are composed by polymerization of the evolutionary conserved $\alpha / \beta$ tubulin heterodimers in a head-to-tail association, to create linear protofilaments that have a diameter of $25 \mathrm{~nm}^{(3)}$. Lateral association of 13 protofilaments give rise to the hollow cylindrical shape that characterizes all MTs ${ }^{(3)}$. Microtubule growth includes the dynamic addition of free heterodimers at the tips of microtubules ${ }^{(4)}$. Rapid depolymerization of microtubules results in microtubule shortening, a procedure known as microtubule catastrophe ${ }^{(5)}$. Addition of free heterodimers at the end of the growing MTs is happening in a stochastic way and is responsible for the "dynamic instability" that MTs exhibit ${ }^{(6)}$. Due to their architecture, MTs have polarity: the minus end of MTs, is capped by $\alpha$-tubulin, whereas the plus-end, which also shows higher growth rate and is found to be more dynamic ${ }^{(6)}$, is capped by $\beta$-tubulin. MTs serve as the building blocks to create larger cellular multi-structures with various morphologies and functions: the dynamic bipolar spindle, the complex neuronal array, the nine-fold symmetric array found in cilia and flagella. The building block of all the above cellular structures is the $\alpha / \beta$-tubulin heterodimer ${ }^{(6)}$.

The most intriguing question is how cells, using a rather simple building block ( $\alpha / \beta$-tubulin heterodimer) create a quite rigid construction (MTs) that can perform and control a plethora of rather complicated cellular functions. MTs do not function alone, as they interact with a large repertoire of MT-associated proteins (MAPS) and cellular effectors that modify MT properties controlling their assembly and dynamic nature. Some MAPS bind to plus or minus end altering MT properties non-covalently, whereas a number of cellular effectors chemically modify the tubulin heterodimer, enhancing the heterogeneity of MTs ${ }^{(7)}$. Thus, MTs although they are composed by an evolutionary conserved building block, they have adopted to functionally specialize for a plethora of functions. This functional specialization is based on MT heterogeneity. Cells express a repertoire of tubulin heterodimers and MTs can be assembled by a combination of these heterodimers.

\section{The tubulin isotypes}

The tubulin heterodimer consists of the highly compacted central structural core and the disordered negatively charged C-terminal tails. The main tubulin core is responsible for tubulin polymerization whereas the highly flexible C-terminal tails are facing the exterior of MTs. The human genome encodes for multiple genes for both $\alpha / \beta$-tubulin isotypes (nine for $\alpha$ - and nine for $\beta$-tubulin). Some of the generic $\alpha$-tubulin and $\beta$-tubulin isotypes, are highly conserved among different species, whereas other tubulin isotypes have co-evolved with specific cell types and microtubule functions. As example, $\beta-1$ tubulin has co-evolved with platelets, a cell type involved in blood coagulation; platelets contain a specialized microtubule array called "marginal band" which is enriched in $\beta-1$ tubulin $^{(8)}$. The number of different isotypes that are 
present in a given cell type, increases the heterogeneity of MTs ${ }^{(9)}$.

Among different isotypes, tubulin tails show a significantly lower sequence identity ( $50 \%)$, compared to the highly conserved tubulin core heterodimer that shows $\sim 80-90 \%$ of identity. Most of the isoform sequence variations and post-translational modifications (PTMs) of tubulin occur on the C-terminal tails and not on the main central core of MTs. This suggests that changes at the C-terminal tails of tubulin is "used" to control the biophysical properties of MTs and their interactions with cellular interacting partners, without altering the MTs polymer nature. Structural studies have shown that the C-terminal tails are highly flexible. This flexibility is a regulatory key of MT dynamics, allowing them to be highly accessible by a number of tubulin modifying enzymes.

\section{The C-terminal tail of tubulin: a regulator or simply a disordered region?}

The majority of the $\alpha$-tubulin tails are composed by short stretches of 10-12 residues, compared to the longer and more sequence divergent $\beta$-tubulin tails. It is clear that the length and charge of the tails rather than the actual sequence has been conserved through evolution. The $\mathrm{C}$-terminal tails for both $\alpha / \beta$-tubulin are enriched in glutamate residues, and less so on aspartate residues, both mediating the electronegativity of the tails. Glutamate is a well-known intrinsic disordered-promoting amino-acid, in protein structures. Both tails are poor in order-promoting amino-acids such as isoleucine, tyrosine, leucine and asparagine, whereas the small non-polar alanine and glycine are also found among the electronegative rich tails. Structural studies of MTs lack information of these rather crucial components of the microtubule polymer, are indeed suggesting that they are flexible; there is however evidence they can adopt helical conformations upon interactions with binding partners ${ }^{(10)}$.

Tubulin tails serve as interacting regions between MTs and molecular motors such as dynein and kinesin-1. Studies with tubulin from $S$. cerevisiae suggest a regulatory role for both $\alpha$ and $\beta$ C-terminal tails. Experiments where the tails have been proteolytically removed, showed that both dynein and kinesin-1, exhibit a lower processivity ${ }^{(11,12)}$. The C-terminal tail of $\alpha$-tubulin acts as a break for the kinesin- 2 molecular motor, since its removal increases the velocity and processivity for kinesin- $2^{(13)}$. On the other hand, the presence of only the C-terminal tail of $\beta$-tubulin is sufficient to reverse the change in motility for both kinesin- 2 and dynein.

The importance of the C-terminal tails on MT structure and function is highlighted by the plethora of proteins that recognize and bind onto them. Tubulin tails are recognized and interact by tau and MAP2 ${ }^{(14)}$. Activity of the microtubule severing enzymes spastin and catanin, is dependent on the presence of the C-terminal tails, whereas absence due to proteolytic degradation has an inhibitory role for both enzymes ${ }^{(15,16)}$. 
1 TBA1A

3 TBA1C

4 TBA3C

5 TUBA3D

6 TBA3E

7 TBA4A

8 TBA8

consensus $>70$
2 TBA1B

VGEGMEEGEF S EAREDMAALEKDYEEVGVDSVEGEG E EEGEEY VGEGMEEGEFSEAREDMAALEKDYEEVGVDSVEGEGEEEGEEY VGEGMEEGEFSEAREDMAALEKDYEEVGADSADGED . . EGEEY VGEGMEEGEFSEAREDLAALEKDYERVGVDSVEAEA . EGEEY VGEGMEEGEFSEAREDLAALEKDYEEVGVDSVEAEAE . EGEEY VGEGMEEGEFSEAREDLAALEKDCEEVGVDSVEAEA . EGEAY VGEGMEEGEFSEAREDMAALEKDYEEVGIDSYEDED . . EGEE . VGEGMEEGEFSEAREDLAALEKDYEEVGTDSFEEEN . . EGEEF VGEGMEEGEFSEARED \$AALEKDYEEVGVDS . \# . . . . EGEEY

1 TBB 1

2 TBB2A

3 TBB3

4 TBB4A

5 TBB4B

6 TBB5

7 TBB6

8 TUBB8

consensus $>70$

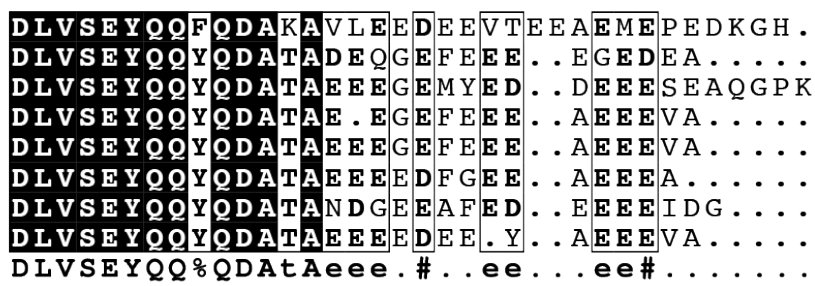

Figure 1. Human C-terminal tubulin tails are highly conserved.

\section{THE TUBULIN CODE}

The term "tubulin code" was introduced in the field to describe the combination of the PTMs of tubulin together with the differential overexpression of tubulin isotypes in cells. The name tubulin code was given based on the analogy with the "histone code", where sequence variability and PTMs are mainly concentrated on the positively charged, $\mathrm{N}$-terminal tails of histones, whereas protomer interfaces are highly conserved ${ }^{(17)}$. Therefore, the tubulin code acts as a molecular programming language that could differentiate microtubules towards distinct functions.

Tubulins are highly conserved macromolecules, and MTs have shown little structural variation during eukaryotic evolution. Their assembly is based on the longitudinal and lateral association of $\alpha$-tubulin and $\beta$-tubulin heterodimers, which restricts the freedom for evolution for the tubulin subunits ${ }^{(2)}$. The variety of $\alpha / \beta$-tubulins, creates a pool of highly conserved but still different heterodimers that can form MTs. The expression and incorporation of multiple isotypes in the MT lattice, has two major effects on MTs: it affects the dynamic stability of MTs since the highly conserved structure of the MTs is slightly altered; it creates variation in the C-terminal regions, affecting MT-MAPS interactions, or PTMs that occur on them ${ }^{(2)}$.

Tubulin post-translational modifications create a repertoire of signals that further differentiate MTs ${ }^{(2)}$. These modifications occur mainly on the $\mathrm{C}$-terminal tails of both $\alpha / \beta$-tubulin. The C-terminal tails are subjected to phosphorylation ${ }^{(18)}$, detyrosination/tyrosination (19-21) glutamylation ${ }^{(22-25)}$, glycylation ${ }^{(26)}$. The structural core of the heterodimer can be acetylated ${ }^{(27}$, ${ }^{28)}$, phosphorylated $^{(29)}$ and polyaminated ${ }^{(30)}$. 
The modifications that are mostly found in tubulin, and particularly at the C-terminal tails are tyrosination, glutamylation and glycynation. All of these include addition of an amino acid residue to a glutamate at the primary sequence of both tubulin tails. During glutamylation or glycylation a $\gamma$-branched isopeptide bond is created, on internal glutamates of both $\alpha / \beta$ tubulin tails ${ }^{(6)}$. Once the first branch has been created, series of glutamates or glycines are added linearly giving rises to short chains that can contain up to 21 glutamates, and up to 34 glycines ${ }^{(26)}$. Short stretches of glutamate residues (polyglutamylation), or glycine residues (polyglycination) occur in both tails. The removal (detyrosination) and ligation of tyrosine (tyrosination) occur on the $\mathrm{C}$-terminal tail of $\alpha$-tubulin. These modifications act as a molecular markers on MTs and are regulated by specific enzymes. The enzymes responsible for the tubulin PTMs, lead to distinct subsets of MTs decorated/programmed to perform certain cellular functions. Both the tubulin heterodimers and MTs can be decorated with PTMs. The modifying enzymes can show preference towards either the MTs or the tubulin heterodimer. Enzymes that detyrosinate, polyglutamylate and acetylate act on MTs, whereas deglutamylases are found to work both on MTs and tubulin heterodimers. Some reverse enzymes, such as the Tubulin Tyrosine Ligase (TTL) decorate only tubulin dimers, providing the unmodified tubulin that can be efficiently incorporated into MTs. All the above suggest that PTMs of tubulin provide mechanisms for the cells to control and regulate necessary cellular procedures. The tubulin code provides cells with a great energetic advantage. The same building blocks can be used and re-modified enabling cells to control vital cellular functions. If we take as an example the cellular procedure of mitosis, the microtubule network is composed by the same building blocks. However, PTMs functionally specialize MTs and further define a molecular pattern that can be recognized by Microtubule associated proteins (MAPs), which will interact with MTs at different stages of the cell cycle. Astral MTs are found to be more tyrosinated compared to interpolar or kinetochore MTs which are mostly detyrosinated. Transportation of chromosomes to the metaphase plate is mediated through Centromere protein-E (CENP-E), which has a preference of migrating on detyrosinated MTs ${ }^{(31)}$. Axonal MTs are found to be more detyrosinated, acetylated and glutamylated whereas MTs of the dynamic growth cone, are mainly tyrosinated ${ }^{(32)}$ Cilia and flagella are enriched in glutamylation, however the MTs in the axonemes of cilia and flagella are highly glycylated ${ }^{(33,34)}$.

It is important to highlight that PTMs do not act as an on/off switch. MTs are highly dynamic polymers that undergo rounds of polymerization and depolymerization. PTMs create a molecular signature that possibly has some degree of periodicity. If we take detyrosination as an example, upon polymerization the tubulin heterodimers that are incorporated are usually tyrosinated, whereas the C-terminal tails of the main body of MTs, are available as substrates for detyrosination. Under specific cellular processes this tyrosination/detyrosination pattern will create the signal for the initiation or even termination of certain protein-protein interactions that are necessary to regulate a cellular function. This highlights how complicated is the interpretation of the tubulin code. The only way to learn the meaning of the tubulin 
code, and if possible, use it in order to "program" certain cellular functions, is by studying the specific enzymes that control these PTMs. Similar to a programming language, understanding the tubulin code can create the necessary tools to create an "in vivo" programming language that could lead to a better understanding or even regulation of certain procedures that take place in cells and usually are deregulated in diseases and cancer.

This thesis focuses in the detyrosination cycle of tubulin, and specifically on the enzymes that remove the C-terminal tyrosine.

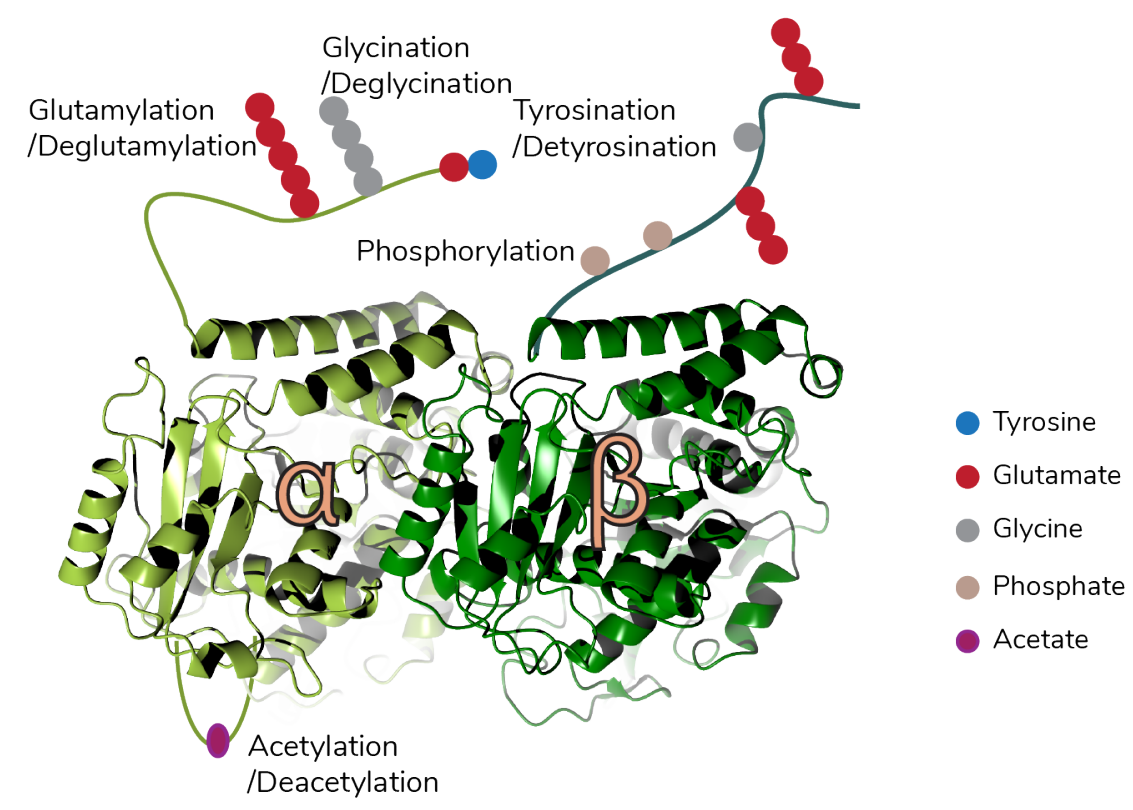

Figure 2. Overview of tubulin PTMs 


\section{THE ROLE OF TUBULIN (DE)TYROSINATION}

Detyrosination has been connected with cellular processes such as mitosis, cardiomyocyte contraction and neuronal development.

During mitosis, cells need to transport chromosomes to the metaphase plate, where chromosomes are aligned before the separation of sister chromatids during the anaphase. MTs regulate the whole procedure by creating the mitotic spindle. This macromolecular assembly is composed by three different types of MTs: interpolar MTs which grow among the two opposing centrosomes, kinetochore MTs which grow from the centrosomes to the kinetochores, and astral MTs which grow from the centrosome to the plasma membrane. All three types of MTs in the mitotic spindle have different tyrosination levels. Astral MTs are found to be more tyrosinated compared to kinetochore and interpolar MTs which are found to be mainly detyrosinated. During prometaphase chromosome transportation takes place by dynein-dynactin, over astral MTs to the spindle pole ${ }^{(35)}$. The next step includes transportation by CENP-E to the metaphase plate, which shows a preference to move over detyrosinated MTs. Therefore, the driving force that leads chromosomes to the metaphase plate is the tyrosination/detyrosination levels of the MTs that compose the mitotic spindle.

Another example that highlights the importance of the detyrosination/ tyrosination status of microtubules that could have a potential in clinical applications, is cardiomyocyte contraction. What makes the heartbeat, is the synchronized contraction of cardiomyocytes. In cardiomyocytes the microtubule network provides mechanical resistance through its interaction with the sarcomere. Upon cardiomyocyte contraction the microtubule network contracts into a sinusoidal configuration ("buckled"). The nature of MTs enables MTs to act as a spring where energy can be stored for the relaxation of the cardiomyocyte ${ }^{(31)}$. Detyrosination acts as a regulating mechanism of this interaction, since alterations in detyrosination levels will lead to stiffness reduction allowing sarcomeres to contract faster. In animal models of heart disease an elevated level of detyrosinatated tubulin is observed ${ }^{(36)}$ which is also characterized by myocyte stiffness and reduced contractility ${ }^{(37)}$. This results in a slower contraction-relaxation cycle compared with healthy subjects. Reducing the rate of detyrosination, simply by overexpressing TTL, will lead to a less dense microtubule network it will lower the stiffness and enhance the contractility.

Neurons, compared to other cell types are highly decorated with PTMs, therefore are an ideal study model to understand the role of PTMs ${ }^{(8)}$. During the maturation process, neurons acquire a number of PTMs. Neuronal $\alpha$-tubulin is found acetylated on Lys40, detyrosinated and further converted to $\Delta-2$ tubulin. Polyglutamylation is another PTM that abundantly occurs, on both $\alpha-/$ $\beta$-tubulin of neuronal MTs ${ }^{(38)}$. Immunofluorescence studies have shown that the axon is mostly acetylated and detyrosinated compared to its growing end, which contains mostly unmodified MTs. The tyrosination/detyrosination ratio in neurons seems to be important for the early stages 
of neuronal development since mice lacking the TTL gene die perinatally ${ }^{(39)}$.

Focusing on neuronal differentiation, knock-down experiments of the enzyme responsible for detyrosination using shRNAs, in combination with an enzymatic inhibitor, reduced the level of detyrosinated $\alpha$-tubulin more than $75 \%$. As a result, a delay in axonal differentiation was observed in neurons cultivated in vitro ${ }^{(40)}$.

The detyrosination/tyrosination cycle also plays an important in the cerebral cortex, of the developing embryonic mouse brain, for the neocortex layer organization ${ }^{(40)}$. In vivo studies in the developing embryonic mouse brain, revealed that radial migration of neurons, depends on detyrosination since a simple knockdown of the detyrosinating enzyme, causes a reduction in differentiation-related migration ${ }^{(31,40)}$. In humans' mutations in SVBP gene, which encodes for the interacting partner protein of the detyrosinating enzyme (chapter-2) are connected to microcephaly and intellectual disability further suggesting an active role of detyrosination in neuronal development ${ }^{(41)}$.

\section{The detyrosination-tyrosination cycle}

Detyrosination is one of the first post-translational modifications described for tubulin (42). It is involved in a number of cellular processes such as mitosis, neuronal differentiation, cardiomyocyte contraction and intracellular transport. Although the detyrosination cycle was always a regulatory element of so many cellular procedures not much attention was given to it, mainly due to lack of biochemical information.

The detyrosination cycle starts by removing the C-terminal tyrosine from the tail of $\alpha$-tubulin free heterodimer, or in the context of polymerized MTs. Experiments conducted in the 1980s highlighted that the detyrosinated/tyrosinated status of MTs affect the MAP-microtubule interactions ${ }^{(43)}$. Later it was shown that localization of CAP-gly containing proteins to the TIP complexes depends on the presence of the EEY/F sequence motif, a characteristic of the tyrosinated form of $\alpha$-tubulin ${ }^{(44)}$. The detyrosination-retyrosination cycle plays an important role in the stability of MTs. Motor proteins of the kinesin family such a kinesin-13 promote microtubule depolymerization which is depended on the presence of tyrosinated tubulin, since detyrosination inhibits the MCAK depolymerization of MTs ${ }^{(45)}$. Other proteins show higher affinities for detyrosinated MTs suggesting the active role of the detyrosination-tyrosination cycle in MT function and dynamics. Kinesin-2 and not Kinesin-1 shows increased motility on detyrosinated MTs ${ }^{(13)}$.

The discovery of tubulin Tyrosine ligase (TTL) as the enzyme responsible for the addition of a free Tyrosine to the $\mathrm{C}$-terminal position of $\alpha$-tubulin was the initiating step of the tubulin code field. Structure determination of TTL shed light on how the enzyme tyrosinates free heterodimers but still the question of how the detyrosination cycle starts was unanswered for almost four decades. 




Figure 3. The detyrosination cycle. MTs are detyrosinated by an unknown enzyme and can also go through MT catastrophe releasing free tubulin heterodimers. Subsequently tubulin heterodimers can be tyrosinated by TTL and then can polymerize into MTs, completing one round of the detyrosination/tyrosination cycle.

\section{Tubulin Tyrosine Ligase is responsible for tubulin tyrosination}

From a historical perspective tyrosination of tubulin is of great importance since it is the first PTM described for tubulin ${ }^{(19)}$. The most important finding was that the incorporation of tyrosine occurs in an RNA independent enzymatic way and takes place in the soluble fraction of rat brain homogenate. Using a rather simple (according to nowadays technological advancements) but elegant approach they identify not only the substrate, which was proved to be tubulin but also all the necessary components of the tyrosination reaction $\left(\mathrm{Mg}^{2+}\right.$, ATP, $\left.\mathrm{K}^{+}\right)^{(19)}$. A few years later the structural and biochemical characterization of Tubulin Tyrosine Ligase ${ }^{(21,46)}$ set the basis for the tubulin code field leading to scientific endeavor from different 
groups to identify the necessary enzymes responsible for different PTMs. The importance of tyrosination is highlighted by the drastic effect that mice lacking TTL die perinatally due to disorganized neuronal arrays ${ }^{(39)}$.

TTL catalyzes for the ATP-dependent addition of a tyrosine residue at the C-terminal tail of $\alpha$-tubulin. The enzyme tyrosinates preferably free tubulin heterodimers and does not act on MTs. Determination of the crystal structure revealed that tubulin heterodimer, when it is in its free form adopts a specific curved conformational change that allows TTL binding and subsequently tyrosination of tubulin. Upon MT polymerization tubulin adopts the "straight" conformation which interferes with TTL binding ${ }^{(47)}$.

The structure of TTL adopts a well-conserved elongated shape that can be subdivided into three main domains ( $\mathrm{N}$-terminal/central/C-terminal). All the three domains participate in the formation of the active site, which also includes an adenoside nucleotide molecule. The distinct structural fold is characteristic of the TTL-like (TTLL) family of proteins, including also polyglutamylases and polyglycylaces ${ }^{(47)}$.

The majority of $\alpha$-tubulin isotypes are genes encoded with a $\mathrm{C}$-terminal tyrosine with the exception of TUBA8 and TUBA4A, containing a terminal phenylalanine and a terminal glutamate, respectively. It is rather obvious that tyrosination of tubulin is taking place in a cyclic way, where the event of detyrosination is the first and triggering step of the second part of the cycle, the addition of tyrosine in the C-terminal tail of tubulin.

a



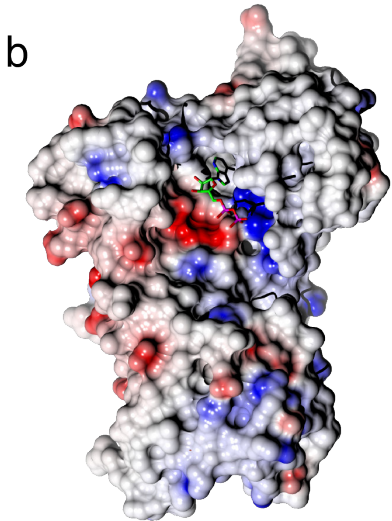

Figure 4. Crystal structure of TTL bound to AMPPNP as ribbons (a) and colored according to electrostatic potential (b).

\section{Vasohibins are responsible for tubulin detyrosination}

The discovery and structure determination of TTL set the basis to understand in molecular detail the role of tyrosination. However, the detyrosination step that initiates this cyclic PTM 
of $\alpha$-tubulin, had remained elusive for almost four decades. The discovery of Vasohibins as the enzymes responsible for the initiating step of detyrosination not only answered this long-standing question in the field but also open a new window to further understand the tubulin code and its importance ${ }^{(40,48)}$. Two independent research groups characterized the role of vasohibins as carboxypeptidases almost 40 years later than the initial discovery of detyrosination.

In chapter-2 we describe the steps leading to the discovery of vasohibins (VASH1 and VASH2) as the main tubulin detyrosinating enzymes, and based on biochemical assays we show that the active detyrosinating enzyme is the complex between vasohibins and their interacting partner the small vasohibin binding protein (SVBP).

In humans two paralogs VASH1 and VASH2 exist: VASH1 encodes for a 365-residue protein and VASH2 for a 355-residue protein. Direct comparison of the two protein sequences reveals a highly conserved structural core and less conserved and rather disordered $\mathrm{N}$-and C- termini. The product of CCDC23 encoding for a small 66 residue polypeptide was identified and named small vasohibin binding protein SVBP ${ }^{(52,53)}$. Indeed, SVBP acts as a binding partner for both VASH1 and VASH2 exhibiting a chaperone-like role. Co-expression of SVBP with both vasohibins improves their solubility and activity ${ }^{(40,48)}$.

Expression of VASHs is ubiquitous in cells but at different basal levels, although in certain cell types one of the two VASHs is expressed ${ }^{(31)}$. Circulating mononuclear cells (MNCs) express VASH2 but not VASH1, whereas endothelial cells show high expression levels for VASH1. Interestingly vasohibins could be found both intracellular and in the cytosol ${ }^{(40,31,50)}$. Intracellular VASH1 did not localize at the endoplasmic reticulum, was not found glycosylated and did not contain a secretion signaling peptide. All the above suggested that VASH1 secretion is based on an ER-independent unconventional pathway ${ }^{(50)}$.

Before the enzymatic classification of vasohibins as tyrosine carboxypeptidases they were studied in the context of angiogenesis. VASH1 was characterized as an inhibitor of angiogenesis, since application of exogenous VASH1 inhibited the proliferation and migration of endothelial cells (ECS), whereas VASH2 promoted angiogenesis and showed high expression in the sprouting front of infiltrated $\mathrm{MNCs}^{(49)}$. The above findings suggested a contradicting role for the two enzymes; despite the medium similarity that the two proteins share overall ( $\sim 5 \%$ identity), the central core where the catalytic triad resides is highly conserved. However, the role of vasohibins in angiogenesis has raised more questions than provided answers. It is rather unclear how vasohibins are secreted from the cells, and there is no evidence so far regarding a specific receptor for vasohibins. Till now there are no data supporting the internalization for either vasohibins by target cells. Taking all the above into consideration, based on our findings we suggest that the main role of vasohibins is as tubulin carboxypeptidases, regulating the detyrosination cycle the detyrosination cycle. 
The lack of structural information and possible substrates was a major reason that the enzymatic nature of vasohibins was not very well understood. A bioinformatics analysis based on protein sequences suggested the presence of a possible catalytic triad (Cys-His-Ser) in both vasohibins ${ }^{(51)}$. Sequence similarity with the Cys-like protease from Cytophaga hutsinsonii suggested that vasohibins are unidentified members of the transglutaminase-like Cys protease superfamily. This family includes members with a diverse catalytic activity, such as proteases, transglutaminases, $\mathrm{N}$-arylamine acetylatransferases. Therefore, it was rather difficult to predict substrates for vasohibins. However, the bioinformatic analysis provided a starting hypothesis regarding the active site of vasohibins, which was proved to be catalytic for the biochemical characterization and classification as tubulin carboxypeptidases ${ }^{(40,48)}$.

The discovery of vasohibins as tyrosine carboxypeptidases, controlling the detyrosination cycle, occurred simultaneously by two different research groups, following completely different approaches. Aillaud et al followed a biochemical-based approach, where by using radiolabelled MTs, they followed carboxypeptidase activity in different fractions of mouse brain homogenate. By fractionation, they strongly enriched for carboxypeptidase activity, and in combination with click chemistry they designed an inhibitor for detyrosination. VASH1 was confirmed to be the tubulin detyrosinating enzyme by mass spectrometry.

Nieuwenhuis et al followed an approach based on haploid genetics to discover the elusive regulating protein of detyrosination. Mutagenized HAP1 cells were treated with paclitaxel, a well-known microtubule stabilizing agent, and stained with antibodies that would recognize for detyrosination. Upon fluorescent activated cell shorting (FACS) two main populations were separated, enriched in low and high level of detyrosination. Identification of SVBP as a positive regulator detyrosination was indeed a breakthrough as it suggested that the complex of VASH1/2 with SVBP is the enzyme responsible for detyrosination of $\alpha$-tubulin. Experiments in cells showed that VASH1/SVBP detyrosinates tubulin heterodimers but has a higher activity on MTs.

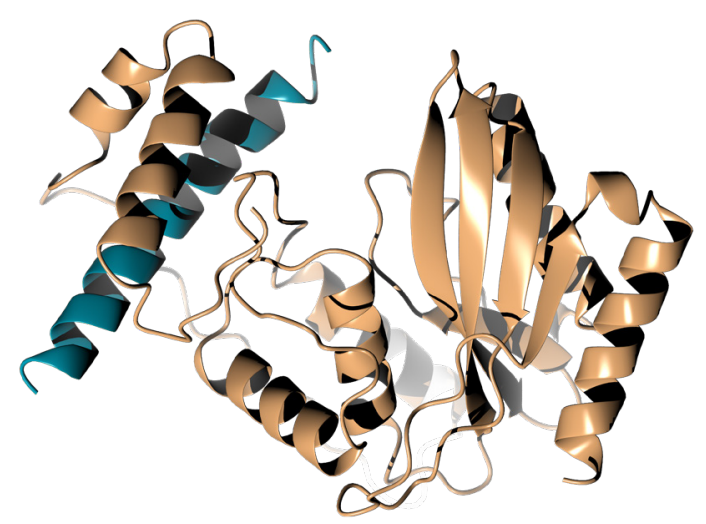

Figure 5. Crystal structure of VASH1/SVBP complex. SVBP colored in teal folds as a helix, stabilizing the main structural core of VASH1 (colored in light orange). 
In chapter 3, we set to understand further the mechanism of detyrosination by VASH1 and the role of SVBP in the complex. We therefore determined the crystal structure of an inactive and truncated VASH1/SVBP complex by X-ray crystallography, and studied the full-length complex by small angle X-ray scattering (SAXS). We identified the active site residues, and then used molecular docking with the high ambiguity driven docking (HADDOCK) software, to gain structural insights on substrate binding and recognition of the C-terminal tubulin tail. To validate the results from the molecular docking we used cell-based assays that further improved and subsequently confirmed our proposed binding model for the tyrosinated tubulin tail onto the VASH1 active site.

During the discovery of vasohibins it was clear that cells contain additional detyrosinating enzymes. Following a similar genetic approach but on a vasohibin-deficient background, Lisa Landskron identified a novel Microtubule Carboxypeptidase (MATCAP), completing the detyrosination repertoire.

In chapter 4 we present the structural characterization of MATCAP using SAXS and X-ray crystallography of the MATCAP catalytic domain (MATCAP-CD) and together with biochemical assays we show that is a metal-dependent carboxypeptidase.

We conclude with chapter $\mathbf{5}$ where we present a general discussion about this thesis. 


\section{REFERENCES}

1. A. F. Pegoraro, P. Janmey, D. A. Weitz, Mechanical Properties of the Cytoskeleton and Cells. Cold Spring Harb Perspect Biol. 9, a022038 (2017).

2. S. Gadadhar, S. Bodakuntla, K. Natarajan, C. Janke, The tubulin code at a glance. J. Cell. Sci. 130, 1347-1353 (2017).

3. C. P. Garnham, A. Roll-Mecak, The chemical complexity of cellular microtubules: tubulin post-translational modification enzymes and their roles in tuning microtubule functions. Cytoskeleton (Hoboken). 69, 442-463 (2012).

4. N. Gudimchuk, A. Roll-Mecak, Watching microtubules grow one tubulin at a time. Proc. Natl. Acad. Sci. U.S.A. 116 7163-7165 (2019).

5. M. K. Gardner, M. Zanic, J. Howard, Microtubule catastrophe and rescue. Curr. Opin. Cell Biol. 25, 14-22 (2013).

6. A. Roll-Mecak, Intrinsically disordered tubulin tails: complex tuners of microtubule functions? Semin. Cell Dev. Biol. 37, 11-19 (2015).

7. A. Akhmanova, M. O. Steinmetz, Control of microtubule organization and dynamics: two ends in the limelight. Nat. Rev. Mol. Cell Biol. 16, 711-726 (2015).

8. C. Janke, M. M. Magiera, The tubulin code and its role in controlling microtubule properties and functions. Nat. Rev. Mol. Cell Biol. 120, 923-20 (2020).

9. S. A. Lewis, W. Gu, N. J. Cowan, Free intermingling of mammalian beta-tubulin isotypes among functionally distinct microtubules. Cell. 49, 539-548 (1987).

10. T. Luchko, J. T. Huzil, M. Stepanova, J. Tuszynski, Conformational analysis of the carboxy-terminal tails of human beta-tubulin isotypes. Biophysical Journal. 94, 1971-1982 (2008).

11. K. S. Thorn, J. A. Ubersax, R. D. Vale, Engineering the processive run length of the kinesin motor. J. Cell Biol. 151, 1093-1100 (2000).

12. Z. Wang, M. P. Sheetz, The C-terminus of tubulin increases cytoplasmic dynein and kinesin processivity. Biophysj. 78, 1955-1964 (2000).

13. M. Sirajuddin, L. M. Rice, R. D. Vale, Regulation of microtubule motors by tubulin isotypes and post-translational modifications. Nat. Cell Biol. 16, 335-344 (2014).

14. P. K. Marya, Z. Syed, P. E. Fraylich, P. A. Eagles, Kinesin and tau bind to distinct sites on microtubules. J. Cell. Sci. 107 ( Pt 1), 339-344 (1994).

15. J. J. Hartman, R. D. Vale, Microtubule disassembly by ATP-dependent oligomerization of the AAA enzyme katanin. Science. 286, 782-785 (1999).

16. A. Roll-Mecak, R. D. Vale, The Drosophila homologue of the hereditary spastic paraplegia protein, spastin, severs and disassembles microtubules. Curr. Biol. 15, 650-655 (2005).

17. T. Jenuwein, C. D. Allis, Translating the histone code. Science. 293, 1074-1080 (2001).

18. B. A. Eipper, Rat brain microtubule protein: purification and determination of covalently bound phosphate and carbohydrate. Proc. Natl. Acad. Sci. U.S.A. 69, 2283-2287 (1972).

19. H. S. Barra, J. A. Rodriguez, C. A. Arce, R. Caputto, A soluble preparation from rat brain that incorporates into its own proteins ( $14 \mathrm{C}$ )arginine by a ribonuclease-sensitive system and ( $14 \mathrm{C}$ )tyrosine by a ribonuclease-insensitive system. J. Neurochem. 20, 97-108 (1973).

20. C. A. Arce, J. A. Rodriguez, H. S. Barra, R. Caputo, Incorporation of L-tyrosine, L-phenylalanine and L-3,4dihydroxyphenylalanine as single units into rat brain tubulin. Eur. J. Biochem. 59, 145-149 (1975).

21. D. Raybin, M. Flavin, An enzyme tyrosylating alpha-tubulin and its role in microtubule assembly. Biochem. Biophys. Res. Commun. 65, 1088-1095 (1975).

22. V. Redeker, J. P. Le Caer, J. Rossier, J. C. Promé, Structure of the polyglutamyl side chain posttranslationally added to alpha-tubulin. J. Biol. Chem. 266, 23461-23466 (1991).

23. V. Redeker, R. Melki, D. Promé, J. P. Le Caer, J. Rossier, Structure of tubulin C-terminal domain obtained by subtilisin treatment. The major alpha and beta tubulin isotypes from pig brain are glutamylated. FEBS Lett. 313, 185-192 (1992).

24. J. E. Alexander et al., Characterization of posttranslational modifications in neuron-specific class III beta-tubulin by mass spectrometry. Proc. Natl. Acad. Sci. U.S.A. 88, 4685-4689 (1991).

25. B. Eddé et al., Posttranslational glutamylation of alpha-tubulin. Science. 247, 83-85 (1990).

26. V. Redeker et al., Polyglycylation of tubulin: a posttranslational modification in axonemal microtubules. Science. 266, 1688-1691 (1994).

27. K. Greer, H. Maruta, S. W. L'Hernault, J. L. Rosenbaum, Alpha-tubulin acetylase activity in isolated Chlamydomonas 
flagella. J. Cell Biol. 101, 2081-2084 (1985).

28. S. W. L'Hernault, J. L. Rosenbaum, Chlamydomonas alpha-tubulin is posttranslationally modified by acetylation on the epsilon-amino group of a lysine. Biochemistry. 24, 473-478 (1985).

29. A. Fourest-Lieuvin et al., Microtubule regulation in mitosis: tubulin phosphorylation by the cyclin-dependent kinase Cdk1. Mol. Biol. Cell. 17, 1041-1050 (2006).

30. Y. Song et al., Transglutaminase and polyamination of tubulin: posttranslational modification for stabilizing axonal microtubules. Neuron. 78, 109-123 (2013).

31. J. Nieuwenhuis, T. R. Brummelkamp, The Tubulin Detyrosination Cycle: Function and Enzymes. Trends Cell Biol. 29, 80-92 (2019).

32. G. Liao, G. G. Gundersen, Kinesin is a candidate for cross-bridging microtubules and intermediate filaments. Selective binding of kinesin to detyrosinated tubulin and vimentin. J. Biol. Chem. 273, 9797-9803 (1998).

33. M. H. Bré et al., Axonemal tubulin polyglycylation probed with two monoclonal antibodies: widespread evolutionary distribution, appearance during spermatozoan maturation and possible function in motility. J. Cell. Sci. 109 ( Pt 4), 727-738 (1996).

34. N. Levilliers, A. Fleury, A. M. Hill, Monoclonal and polyclonal antibodies detect a new type of post-translational modification of axonemal tubulin. J. Cell. Sci. 108 ( Pt 9), 3013-3028 (1995).

35. M. Barisic, P. Aguiar, S. Geley, H. Maiato, Kinetochore motors drive congression of peripheral polar chromosomes by overcoming random arm-ejection forces. Nat. Cell Biol. 16, 1249-1256 (2014).

36. P. Robison et al., Detyrosinated microtubules buckle and bear load in contracting cardiomyocytes. Science. $\mathbf{3 5 2}$ aaf0659-aaf0659 (2016).

37. C. Y. Chen et al., Suppression of detyrosinated microtubules improves cardiomyocyte function in human heart failure. Nat. Med. 24, 1225-1233 (2018).

38. F. J. Ahmad, T. P. Pienkowski, P. W. Baas, Regional differences in microtubule dynamics in the axon. J. Neurosci. 13, 856-866 (1993).

39. C. Erck et al., A vital role of tubulin-tyrosine-ligase for neuronal organization. Proc. Natl. Acad. Sci. U.S.A. 102, 7853-7858 (2005).

40. C. Aillaud et al., Vasohibins/SVBP are tubulin carboxypeptidases (TCPs) that regulate neuron differentiation. Science. 358, 1448-1453 (2017).

41. Z. Iqbal et al., Loss of function of SVBP leads to autosomal recessive intellectual disability, microcephaly, ataxia, and hypotonia. Genet. Med. 21, 1790-1796 (2019).

42. M. E. Hallak, J. A. Rodriguez, H. S. Barra, R. Caputto, Release of tyrosine from tyrosinated tubulin. Some common factors that affect this process and the assembly of tubulin. FEBS Lett. 73, 147-150 (1977).

43. N. Kumar, M. Flavin, Modulation of some parameters of assembly of microtubules in vitro by tyrosinolation of tubulin. Eur. J. Biochem. 128, 215-222 (1982).

44. A. Weisbrich et al., Structure-function relationship of CAP-Gly domains. Nat. Struct. Mol. Biol. 14, 959-967 (2007)

45. L. Peris et al., Motor-dependent microtubule disassembly driven by tubulin tyrosination. J. Cell Biol. 185, 11591166 (2009).

46. D. Raybin, M. Flavin, Enzyme which specifically adds tyrosine to the alpha chain of tubulin. Biochemistry. 16 2189-2194 (1977).

47. A. E. Prota et al., Structural basis of tubulin tyrosination by tubulin tyrosine ligase. J. Cell Biol. 200, 259-270 (2013).

48. J. Nieuwenhuis et al., Vasohibins encode tubulin detyrosinating activity. Science. 358, 1453-1456 (2017).

49. H. Kimura et al., Distinctive localization and opposed roles of vasohibin-1 and vasohibin-2 in the regulation of angiogenesis. Blood. 113, 4810-4818 (2009).

50. T. Shibuya et al., Isolation and characterization of vasohibin-2 as a homologue of VEGF-inducible endotheliumderived angiogenesis inhibitor vasohibin. Arterioscler. Thromb. Vasc. Biol. 26, 1051-1057 (2006).

51. L. Sanchez-Pulido, C. P. Ponting, Vasohibins: new transglutaminase-like cysteine proteases possessing a noncanonical Cys-His-Ser catalytic triad. Bioinformatics. 32, 1441-1445 (2016).

52. Y. Suzuki et al., Isolation of a small vasohibin-binding protein (SVBP) and its role in vasohibin secretion. J. Cell. Sci. 123, 3094-3101 (2010).

53. Y. Sato, The vasohibin family: a novel family for angiogenesis regulation. J. Biochem. 153, 5-11 (2013). 



\section{Chapter 2}

\section{Vasohibins encode tubulin detyrosinating activity}

Joppe Nieuwenhuis ${ }^{1}$, Athanassios Adamopoulos ${ }^{1}$, Onno B. Bleijerveld ${ }^{1}$, Abdelghani Mazouzi ${ }^{1}$, Elmer Stickel ${ }^{1}$, Patrick Celie ${ }^{1}$, Maarten Altelaar ${ }^{1,2}$, Puck Knipscheer $^{3}$, Anastassis Perrakis ${ }^{1}$, Vincent A. Blomen, Thijn ${ }^{1^{*}}$ R. Brummelkamp ${ }^{1,4,5^{*}}$

Affiliations:

${ }^{1}$ Division of Biochemistry, Netherlands Cancer Institute, Plesmanlaan 121, 1066 CX, Amsterdam, The Netherlands.

${ }^{2}$ Biomolecular Mass Spectrometry and Proteomics, Utrecht Institute for Pharmaceutical Sciences, University of Utrecht, Padualaan 8, 3584CH Utrecht, The Netherlands.

${ }^{3}$ Hubrecht Institute, Royal Netherlands Academy of Arts and Sciences, Uppsalalaan 8, 3584 CT, Utrecht, The Netherlands. ${ }^{4}$ CeMM Research Center for Molecular Medicine of the Austrian Academy of Sciences, 1090 Vienna, Austria.

${ }^{5}$ CGC.nl, Plesmanlaan 121, 1066CX, Amsterdam, The Netherlands.

*Contact information: correspondence and requests for materials should be addressed to V.A.B. (vincent.blomen@ scenicbiotech.com) or T.R.B. (t.brummelkamp@nki.nl).

\section{Based on:}

Science, 2017, 358 (6369),1453-1456. 


\section{ABSTRACT}

Tubulin is subjected to a number of posttranslational modifications, referred to as 'the tubulin code', enabling the generation of heterogeneous microtubules. This includes a unique cycle of removal and ligation of the carboxy-terminal tyrosine of $\alpha$-tubulin. Whereas dedicated enzymes have been assigned to most tubulin modifications, the enzymes responsible for detyrosination, an activity observed forty years ago, remained elusive. We applied a haploid genetic screen to find regulators of tubulin detyrosination and identified SVBP, a peptide that regulates the abundance of Vasohibins (VASH1 and VASH2). Vasohibins, but not SVBP alone, increase the levels of detyrosinated $\alpha$-tubulin and we confirm that purified Vasohibins specifically remove the carboxy-terminal tyrosine of $\alpha$-tubulin. Genetic disruption demonstrates a cell-type dependent critical function of Vasohibins in tubulin detyrosination, but also highlights the existence of additional detyrosinating activity. Thus Vasohibins, hitherto studied as secreted factors regulating angiogenesis, constitute a long-sought missing link in the tubulin tyrosination cycle. 
Microtubules are crucial constituents of the eukaryotic cytoskeleton, a dynamic structure important for cell shape and intracellular transport, composed of polymerized $\alpha$ - and $\beta$-tubulin heterodimers. Extensive enzymatic alterations create heterogeneous microtubules decorated with a variety of posttranslational modifications including acetylation, (poly)glutamylation, (poly)glycylation and polyamination ${ }^{(1)}$. Most $\alpha$-tubulin isoforms encode a tyrosine at their carboxy-terminus that can be proteolytically removed and re-ligated. The incorporation of tyrosine, the first described posttranslational modification of tubulin, is carried out by the Tubulin Tyrosine Ligase (TTL) which reverses the detyrosinated $\alpha$-tubulin state to the translated form ${ }^{(2)}$. However, the activity of the detyrosinating enzyme ${ }^{(3)}$, which initiates the tyrosination cycle, remains unclear.

Tubulin detyrosination has been implicated in cardiac cell function ${ }^{(4)}$, cell migration ${ }^{(5)}$, mitosis ${ }^{(6)}$, and trafficking in neurons ${ }^{(7)}$. We applied a genetic approach using haploid human cells ${ }^{(8,9)}$ to identify tubulin-detyrosinating enzymes. Detyrosinated $\alpha$-tubulin could be detected in wildtype Hap1 cells, and this signal was increased in TTL-deficient HAP1 cells and cells treated with the microtubule stabilizing agent paclitaxel (Fig. 1A), indicating that the tyrosination cycle is active in HAP1 cells. Next, mutagenized HAP1 cells were stained with antibodies recognizing the detyrosinated form of $\alpha$-tubulin levels were isolated by fluorescence-activated cell sorting (FACS). Gene-trap insertion sites were mapped to identify genes enriched for mutations in cells exhibiting either high or low levels of $\alpha$-tubulin detyrosination (Fig. 1B) ${ }^{(9)}$. TTL was identified as the strongest negative regulator of $\alpha$-tubulin detyrosination (647 independent gene-trap insertion events mapped in the locus in the "high" cell population versus 11 mutations in the "low" population; Fig. 1C). In addition, we identified both subunits (KATNA1 and KATNB1) of the microtubule-severing protein complex katanin as negative regulators, and CAMSAP2 and MAP4 as positive regulators, in agreement with previous studies ${ }^{(10,11)}$. Among the genes that were enriched by at least a factor of 4 for mutations in the "low" channel, SVBP (small vasohibin binding protein) was identified as the most significant hit ( $P=$ $4 \times 10-10)$. Using an antibody from a different supplier to enrich for cells with high and low levels of tubulin detyrosination, mutations in SVBP were similarly enriched in the population displaying "low" detyrosination ( $n=62$ independent mutations), whereas no mutations in this locus could be identified in the population displaying "high" detyrosination levels (fig. S1A). Neither TTL nor SVBP scored as regulators in 10 unrelated genetic screens examining diverse protein phenotypes (fig. S1B), which suggests that SVBP has a specific function in $\alpha$-tubulin detyrosination.

The interaction of SVBP, encoding a short peptide (66 amino acids), with Vasohibins ${ }^{(12)}$ further suggested a possible function of SVBP in tubulin detyrosination. Although vasohibins have a predicted transglutaminase-like protease fold ${ }^{(13)}$, enzymatic activity has not been demonstrated and substrates have not been proposed. They are found in the cytosol but are considered to act in the extracellular milieu after secretion through a noncanonical pathway ${ }^{(12,14,15)}$. 
Mammalian cells contain two vasohibin paralogs, VASH1 and VASH2 (fig. S1, C and D), that may act redundantly. To assess the function of SVBP and vasohibins in detyrosination, we expressed SVBP, VASH1, and VASH2 in HeLa cells, a cell line with minimal levels of detyrosinated $\alpha$-tubulin ${ }^{(16)}$ (Fig.1D). Whereas SVBP did not increase detyrosinated $\alpha$-tubulin, expression of VASH1 or VASH2 modestly increased detyrosinated $\alpha$-tubulin.

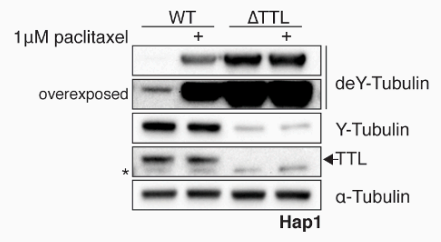

C

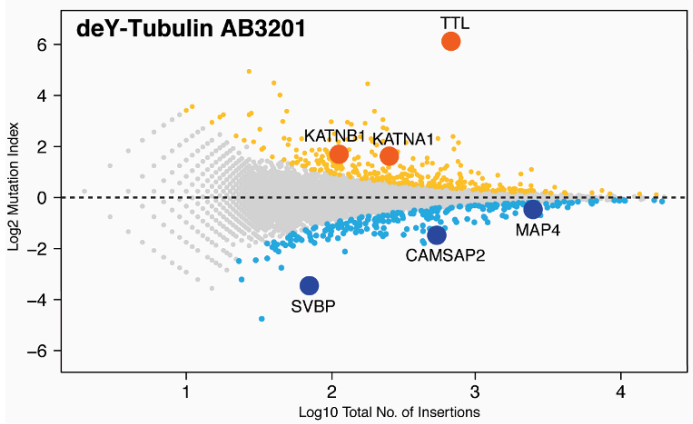

B

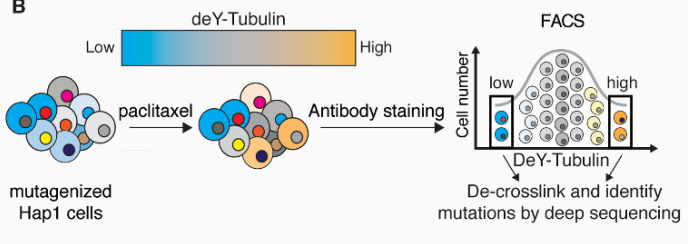

D

Figure 1. Identification of genetic regulators of tubulin detyrosination in haploid human cells. (A) Wild-type Hap1 cells and Hap1 cells deficient for TTL were treated with paclitaxel and subjected to immunoblot analysis using antibodies directed against detyrosinated and tyrosinated tubulin and TTL. Total amounts of tubulin were used as loading control. The relative ratios of detyrosinated vs. total alpha-tubulin levels are indicated. Asterisk indicates that deY-Tubulin signal is not quantifiable. (B) Schematic overview of the haploid genetic screen using antibodies detecting detyrosinated tubulin. (C) Result of the genetic screen for regulators of alpha-tubulin detyrosination. The relative mutation frequency in the 'high' versus the 'low' cell population (referred to as Mutation Index or MI) was plotted against the total amount of insertions mapped per gene. Positive regulators are labeled in yellow, negative regulators in blue. (D) HeLa cells transfected with vectors directing the expression of FLAG-tagged SVBP, VASH1, VASH2, or combinations thereof, were subjected to immunoblot analysis.

Coexpression of SVBP with vasohibins increased the abundance ${ }^{(12)}$ and solubility (fig. S2) of vasohibins and further increased detyrosination of $\alpha$-tubulin. Thus, SVBP together with vasohibins can increase $\alpha$-tubulin detyrosination.

To determine whether endogenous vasohibins affected detyrosination of a-tubulin, we generated single- and double-knockout cell lines (fig. S3). Loss of VASH1 or VASH2 led to a modest decrease in the amount of detyrosinated tubulin in Hap1 cells, and their combined loss led to a further decrease (but not absence) of detyrosinated tubulin (Fig. 2A). The presence of detyrosinated tubulin in vasohibin-deficient cells could not be attributed to expression 
of TUBA4A (fig. S4), an isoform lacking the C-terminal tyrosine. To investigate the role of the vasohibins in other cell types, we generated both VASH1-deficient and VASH1/VASH2-deficient cell lines in human embryonic kidney (HEK) 293T cells (fig. S5) as well as in the melanoma-derived cell line CHL-1 (fig. S6). In HEK293T cells, a minimal decrease in detyrosinated a-tubulin was observed in VASH1 mutant cells, but a substantial decrease was observed in double-knockout cells (Fig. 2B). In CHL-1 cells, however, the double-knockout cells displayed undetectable levels of detyrosinated $\alpha$-tubulin (Fig. 2B) and a modest increase in the amount of tyrosinated tubulin (fig. S7A). Thus, vasohibins are important for $\alpha$-tubulin detyrosination and cells can also contain vasohibin-independent detyrosinating activity. Having identified vasohibin-dependent and-independent activities, we next sought to determine whether these could affect the polymerized microtubule population. Paclitaxel stabilizes microtubules and thereby depletes the amount of free $\alpha / \beta$-tubulin dimers that are the substrate for TTL ${ }^{(17)}$. Treatment of Hap1, HEK293T, and CHL-1 cells with paclitaxel led to a robust increase in $\alpha$-tubulin detyrosination. In Hap1 and HEK293T cells deficient for VASH1 and VASH2, a similar response was observed; this was also the case when translation was inhibited by cycloheximide in HEK293T cells (Fig. $2 \mathrm{C}$ and Fig. S8). These findings suggest that the vasohibin-independent activity affects paclitaxel-stabilized microtubules. Comparative immunoblot analysis showed that vasohibins mediate at least $97 \%$ of $\alpha$-tubulin detyrosination in $\mathrm{CHL}-1$ cells (fig. S9). Paclitaxel treatment also increased detyrosination in these cells, which suggests that vasohibins also affect the detyrosination status of polymerized microtubules. To address this further, we stained CHL-1 cells using antibodies directed against $\alpha$-tubulin and tyrosinated and detyrosinated tubulin (Fig. 2D and fig. S7B). The signal for detyrosinated tubulin was absent in vasohibin-deficient cells, but it colocalized with microtubules in both interphase and mitotic wild-type cells ( Fig. 2D and fig. S10). Thus, vasohibins affect the detyrosination state of polymerized microtubules, although their activity appeared not to be absolutely needed for chromosome congression ${ }^{(6)}$.

To directly test whether vasohibins act as transglutaminase peptidases toward tyrosinated $\alpha$-tubulin, we designed VASH1 and VASH2 mutants that affected their respective predicted catalytic sites, $\mathrm{Cys}^{169}$ and $\mathrm{Cys}^{93}{ }^{933}$. Coexpression of VASH1-Cys169Ala and VASH2-Cys93Ala with SVBP showed that these cysteines were essential for vasohibin-dependent induction of detyrosinated $\alpha$-tubulin (Fig. 3A).

To study whether vasohibins can produce detyrosinated $\alpha$-tubulin in vitro, we coexpressed the VASH1-SVBP complex in insect cells (Fig. 3B) and purified a stable and soluble complex. Smallangle $x$-ray scattering (SAXS) coupled to size exclusion chromatography revealed a well-folded, structurally robust, elongated complex with 1:1 stoichiometry (fig. S11). Purified VASH1-SVBP reduced the tyrosinated form of tubulin while increasing the detyrosinated form as examined by specific antibodies, with an apparent Michaelis constant $K_{\mathrm{M}}$ of $\sim 700 \mathrm{nM}$ on $\alpha / \beta$-tubulin (fig. S12). VASH1 alone was expressed in small amounts, again suggesting that SVBP is needed for folding, and thus solubility, of VASH1. 


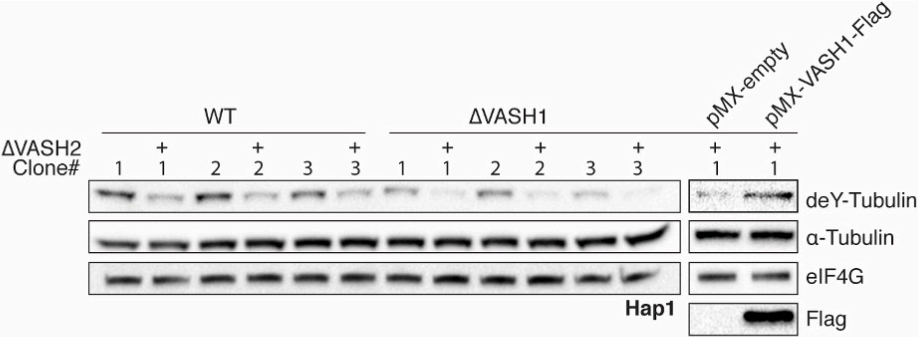

B

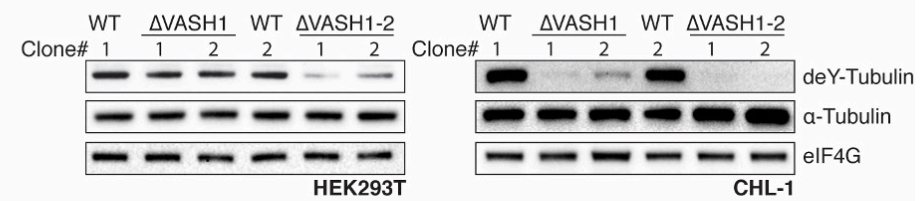

C

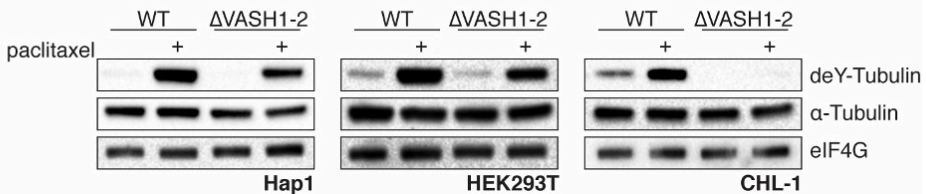

D

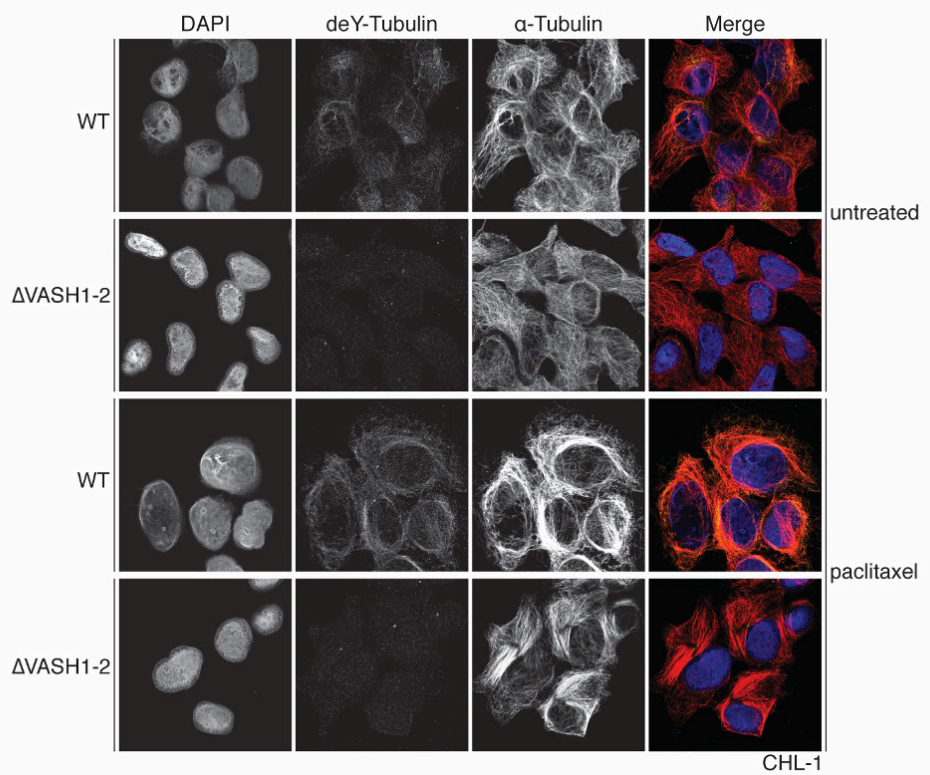

Figure 2. VASH1 and VASH2 control tubulin detyrosination and affect the detyrosination status of polymerized microtubules. (A) Independent Hap1 cell lines deficient for VASH1- or VASH1 and VASH2 were generated and subjected to immunoblot analysis. The relative ratios of detyrosinated vs. total alpha-tubulin levels are indicated. (B) Independent clonal HEK293T and CHL-1 cell lines deficient for VASH1 and/or VASH2 were generated and subjected to immunoblot analysis (C) Wild-type cells and cell lines deficient for VASH1 and VASH2 were treated with paclitaxel and subjected to immunoblot assay as in (A). (D) Wild-type CHL-1 cells and CHL-1 cells deficient for VASH1 and VASH2 were treated with paclitaxel and stained with antibodies to detect detyrosinated alpha-tubulin (green) and alpha-tubulin (red). Blue indicates 4',6-diamidino-2-phenylindole (DAPI) nuclear counterstain. Paclitaxel treatment led to a factor of 1.89 increase in the detyrosination/total tubulin ratio of wild-type cells. Scale bars, $25 \mu \mathrm{m}$. 
Relative to nontreated $\alpha / \beta$-tubulin, the in vitro detyrosination rate of VASH1-SVBP was higher using guanosine triphosphate (GTP)-induced polymerized stabilized microtubules as a substrate by a factor of $\sim 2.5(P=0.013)$ (Fig. 3D) Whereas immunoblot analysis suggested that detyrosinated $\alpha$-tubulin is generated by vasohibins, it is a possibility that other reaction products could also be generated, including the deglutaminated $\Delta 2$ - or $\Delta 3$-forms of $\alpha$-tubulin ${ }^{(17}$, ${ }^{18)}$. Nano-liquid chromatography combined with tandem mass spectrometry (nanoLC-MS/MS) indicated that VASH1-SVBP detyrosinated $\alpha$-tubulin without affecting the adjacent glutamic acid residues (Fig. 3E). Thus, VASH1 acts as a peptidase to catalyze removal of the C-terminal tyrosine of $\alpha$-tubulin.

We next designed experiments to study the specificity of vasohibins. Tubulin isoforms encode different $\mathrm{C}$-terminal tails. These tails were attached to the $\mathrm{C}$ terminus of green fluorescent protein (GFP) and coexpressed with VASH1, VASH1-Cys169Ala, and VASH2. All isoforms containing a tyrosine at their $\mathrm{C}$ terminus could be detyrosinated; TUBA8A, which encodes for a C-terminal phenylalanine, could also be modified by vasohibins (fig. S13). To further determine the substrate specificity, we generated mutants in the TUBA1A/B minimal substrate. Only variants with a C-terminal tyrosine or phenylalanine were processed by vasohibins (Fig. 3F), suggesting a requirement for an aromatic ring at the $\mathrm{C}$-terminal position. Extension of the $\mathrm{C}$ terminus with a glycine prevented enzymatic conversion by vasohibins, suggesting that the terminal free carboxyl group is required and that vasohibins do not cleave internally. These experiments start to provide a rationale for the specific proteolysis of the $C$ terminus of $\alpha$-tubulin.

Previously, vasohibins have been studied as secreted molecules affecting angiogenesis, although the mechanism of secretion remains unclear ${ }^{(19,20,21)}$. The enzymatic activity described here addresses long-standing questions about the nature of molecules that are able to start the detyrosination-tyrosination cycle. Additional studies are required to address whether certain isoforms or modified versions of vasohibins function specifically inside the cell to detyrosinate tubulin. Although detyrosination was envisioned as a simple reaction carried out by a carboxypeptidase, the identification of VASH1, VASH2, the regulating peptide SVBP, and a yet-unidentified activity extend our view on the complexity of this process. 



$\mathbf{E}$
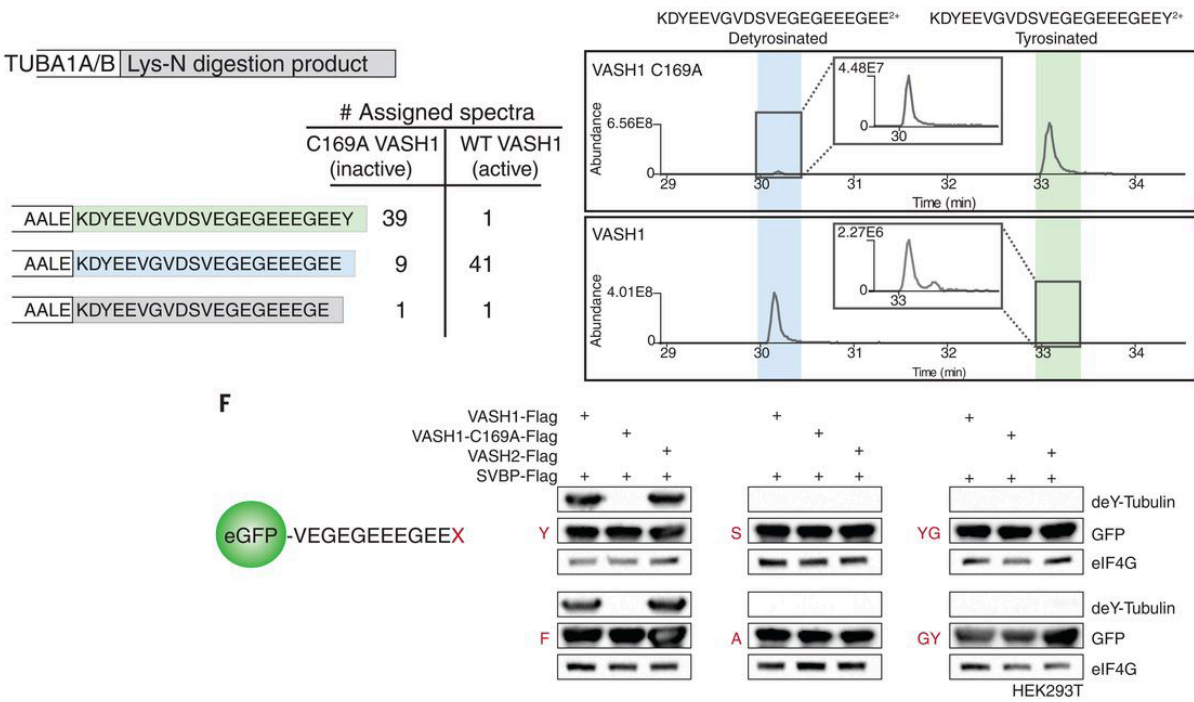

Figure 3. The catalytic activity of Vasohibins specifically removes the tyrosine residue of alpha-tubulin. (A) HeLa cells were transfected with indicated plasmids and subjected to immunoblot analysis. (B) Coomassie staining of a gel loaded with the purified products of SVBP, SVBP:VASH1 and SVBP:VASH1-C169A expressed in insect cells. (C) In vitro detyrosination assay, using recombinant SVBP, VASH1, VASH1:SVBP and catalytic inactive VASH1:SVBP using purified HeLa $\alpha / \beta$-tubulin as substrate. Tubulin tyrosination and detyrosination levels were determined using immunoblot analysis. (D) Purified VASH1-SVBP was incubated with in vitro generated microtubules from HeLa cells, and immunoblot signals were quantified to establish the detyrosination rate relative to nontreated $\alpha / \beta$-tubulin $(n=3)$. $(E)$ NanoLCMS/MS analysis of HeLa tubulin incubated with catalytic active or inactive VASH1-SVBP complexes. Extracted ion chromatograms of the detyrosinated and tyrosinated peptides are shown together with the number of assigned spectra of the respective peptides. (F) HEK293T cells were cotransfected with vectors encoding the expression of enhanced GFP molecules with C-terminal extensions corresponding to the $\mathrm{C}$ terminus TUBA1A/B with the indicated modifications as well as SVBP and VASH1, VASH2, or catalytically inactive VASH1. Transfected cells were subjected to immunoblot analysis. Amino acid abbreviations: A, Ala; D, Asp; E, Glu; F, Phe; G, Gly; K, Lys; L, Leu; S, Ser; V, Val; Y, Tyr. 


\section{Acknowledgments}

We thank R. Medema, J. van den Berg, H. Janssen, and members of the Brummelkamp laboratory for discussions; A. Fish for advice in performing biochemical assays; M. StadnikSpiewak for assisting in protein expression experiments; D. Peeper for CHL-1 cells; and T. Sixma for carefully reading the manuscript. Supported by NWO Vici Grant 016.Vici.170.033, KWF grant NKI2015-7609, the Cancer Genomics Center (CGC.nI), and the Ammodo KNAW Award 2015 for Biomedical Sciences (T.R.B.); the Netherlands Organization for Scientific Research (NWO) as part of the National Roadmap Large-scale Research Facilities of the Netherlands, Proteins@Work project number 184.032.201 (O.B.B. and M.A.); and VIDI grant 723.012.102 (M.A.). The HAP1 cell line is available from T.R.B. under a material transfer agreement with the Netherlands Cancer Institute. Sequencing data have been deposited in the NCBI Sequence Read Archive (www.ncbi.nlm.nih.gov.sra) under accession number SRP119153. Processed screen results are accessible in an interactive database (https://phenosaurus.nki.nl/).

\section{Supplementary materials}

Other Supplementary Materials for this manuscript includes the following:

Table S1 (available online) 


\section{MATERIALS AND METHODS}

\section{Cell lines and culture}

Hap1 cells were cultured in IMDM-medium (ThermoFisher Scientific) supplemented with $10 \%$ heat-inactivated fetal calf serum (FCS; Sigma Aldrich) and streptomycin-glutaminepenicillin (ThermoFisher Scientific). CHL-1 and HEK293T cells were cultured in DMEM-medium (ThermoFisher Scientific), supplemented with 10\% heat-inactivated FCS and streptomycinglutamine-penicillin (ThermoFisher Scientific). All cell lines were regularly checked for mycoplasma and verified to be negative.

\section{Screen for genetic regulators of detyrosinated $\alpha$-tubulin}

The haploid genetic screen was carried out as described before in detail in ${ }^{(1)}$. In brief: $4 \mathrm{e}^{9}$ mutagenized Hap1 cells were treated with $2 \mu \mathrm{M}$ paclitaxel (Teva UK Limited) for 3 hours. Cells were harvested, washed with ice-cold PBS followed by fixation with pre-cooled methanol (Sigma Aldrich \#32213) for 10' on ice. Cells were resuspended in PBS (ThermoFisher Scientific) supplemented with $10 \%$ FCS, hereafter called FACS buffer. Cells were subjected to multiple rounds of straining ( $40 \mu \mathrm{m}$ strainer, BD Falcon) to create a single cell suspension which was treated with $10 \mathrm{\mu g} \mathrm{ml}^{-1}$ RNAse A (Qiagen) for $30^{\prime}$ at $37^{\circ} \mathrm{C}$. Of this suspension, $3 \mathrm{e}^{9}$ cells were stained using antibody AB3201 (Merck-Millipore) (ab48389 from Abcam for the second purification, figure S1A) directed against detyrosinated $\alpha$-tubulin, for 2 hours at room temperature (RT). After washing with FACS buffer, secondary antibody Goat-anti-RabbitAlexaFluor-488 (ThermoFisher Scientific \#A-11008) was added for 1 hour at RT. Washing with FACS buffer was followed by $10 \mu \mathrm{g} \mathrm{ml}^{-1}$ Propidum lodide (ThermoFisher Scientific) staining. Sorting was performed on a S3 Sorter (Bio-Rad) until $10 \mathrm{e}^{6}$ cells for both the highest- and lowest $1 \%$ of the detyrosination signal were retrieved. Only G1-cells were gated to prevent the contamination with diploid Hap1 cells. Sorted cells were collected and genomic DNA isolation and library preparation were performed as described in Brockmann et al ${ }^{(1)}$. Briefly, sequence reads were aligned to hg19 tolerating a single mismatch and assigned to non-overlapping protein-coding gene regions (Refseq). The number of in sense gene trap integrations in each gene was compared between the high and low deY-tubulin population by means a two-sided Fisher's exact test (false discovery rate (Benjamini and Hochberg)-corrected). A complete overview of the number of insertion sites mapped in the screen is available in supplementary table 1.

\section{Immunoblotting}

For immunoblot analysis SDS-sample buffer (0.25M Tris-HCl pH 6.8; 6\% SDS, 30\% Glycerol, $16 \% \beta$-mercaptoethanol and bromophenol blue) was added directly to PBS washed cells. SDS gels used are all 4\%-12\% Bis-Tris gels (ThermoFisher Scientific \#NP0355) ran in MOPS buffer (ThermoFisher Scientific \#NP-0001) and blotted on PVDF (Immobilon-P Merck Millipore \#IPVH00010) in standard blotting buffer containing 20\% ethanol. Blocking of the immunoblot 
was done in 4\% milk (Merck Millipore \#1.15363.0500) in TBS-T. Antibodies used for immunoblot analysis: elF4G (Cell Signaling Technologies \#2498); $\alpha$-tubulin (DM1A; Santa Cruz \#32293); detyrosinated $\alpha$-tubulin (Merck Millipore AB3201); TTL (Proteintech 1318-1-AP); Tyrosinated tubulin (YL1/2 Sigma Aldrich MA1 80017); Flag (CST \#2368); GFP (Santa Cruz \#sc8334).

\section{Quantifications of immunoblots}

The ratios of detyrosinated tubulin vs alpha-tubulin levels are presented as relative values compared to the highest ratio found on that particular immunoblot. Quantifications were performed using FIJI (FIJI Is Just Image-J) software. Bands of both total alpha-tubulin and detyrosinated alpha-tubulin were quantified individually and background was determined by placing a square with the same size on a random location on the same immunoblot. The average value of the background was subtracted from the measured intensity. The values obtained by these measurements were used to calculate the ratio of detyrosinated alphatubulin over total alpha-tubulin. Subsequently, all the values were divided by the highest value, giving the relative ratio for an individual sample.

\section{Cycloheximide/paclitaxel treatment and 35S-labeling}

Cycloheximide/paclitaxel treatment. Cells were co-treated with freshly prepared cycloheximide (160 $\mathrm{g} \mathrm{m} \mathrm{ml}^{-1}$ ) for one hour ( in other experiments paclitaxel treatment is used for three hours). Hap1 $\triangle$ VASH1-2 cells were starved in methionine- and cysteine deficient DMEM for 30 minutes. $160 \mu \mathrm{g} \mathrm{ml}-1$ freshly prepared cycloheximide was added for $40 \mathrm{~min} .{ }^{35} \mathrm{~S}$-trans-label was added for 20 minutes. Cells were washed three times with ice-cold PBS to remove free label, then sample buffer was added. Lysates were run ona standard gel (4\%-12\% as mentioned before) and after drying imaged on Typhoon FLA 9500 (GE Healthcare).

\section{Immunofluorescence}

CHL-1 cells were seeded on gelatin-coated cover slips in 12-well plates. After 24 hours, cells were treated with $2 \mu \mathrm{M}$ paclitaxel for 2 hours. After washing three times with ice-cold PBS, cells were fixed and permeabilized with pre-cooled Methanol (Sigma Aldrich \#32213) for 4' and blocked using 5\% normal goat serum (Sigma Aldrich \#G9023) for 1 hour at RT, followed by three washes in PBS supplemented with $1 \%$ goat serum (IF buffer). Cells were stained overnight in a wet chamber at $4^{\circ} \mathrm{C}$ using same antibodies as for immunoblotting in IF buffer. After rinsing with IF buffer, secondary antibody Goat-anti-Rabbit-AlexaFluor-488 (ThermoFisher Scientific \#A-11008) and Goat-anti-Mouse-AlexaFluor-568 (ThermoFisher Scientific \#A-11004) were incubated for one hour at RT in a wet chamber. After washing with IF buffer, slides were incubated with $1 \mathrm{\mu g} \mathrm{ml}^{-1}$ 4',6-diamidino-2-phenylindole (DAPI) for $15^{\prime}$ at RT. Slides were washed and mounted using Shandon Immu-Mount (ThermoFisher Scientific \#9990402) and imaged the same day, on Leica SP5 confocal microscope using LCS software. 


\section{Quantification of immunofluorescence}

To quantify the rate of detyrosination in CHL-1 wild-type and vasohibin deficient cells, the Cellprofiler software ${ }^{(22)}$ was used to determine the pixel area where tubulin is present using the alpha-tubulin images, excluding the nuclear region based on the DAPI signal. The mean of the pixel intensity of the detyrosinated alpha-tubulin signal per cell was determined in the same area.

\section{Generation of knock-out cell lines}

As multiple isoforms and splice-variants of Vasohibins are present, we designed gRNAs targeting the exon encoding the catalytic cysteine in both VASH1 and VASH2 (VASH1: 5' ACGGCTTCCAGGCATTTGAT-3'; VASH2: 5' -TTTGATAGGCAAGGACTCTC-3'). These gRNAs were cloned all in px330, pLentiV2_Blast (addgene \#83480) and pxpr_001.

The Hap1 mutants as displayed in Fig. 2A were obtained as following: the $\triangle$ VASH1 cell line used as background to generate the double knock out created by transfection with px330 VASH1exCatCys and co-transfection with a plasmid containing CMV-driven expression of the Blasticidin resistance gene (CMV-Blasticidin ${ }^{\text {res }}$ ). Resistant cells were subcloned and genomic DNA was isolated using DirectPCR (cell) (Viagen \#302-C). Clonal cell lines were tested for ploidy and diploid clones were disregarded. To generate the $\triangle \mathrm{VASH} 2$ mutants, in parallel, Hap1-WT and $\triangle$ VASH1 cells were transfected with either px330_empty or px330_VASH2exCatCys, cotransfected with the CMV-Blasticidin ${ }^{\text {res }}$ plasmid. After selection, all cell lines were subcloned and subjected to sequencing to identify clones bearing disruptive mutations.

To sequence the affected locus, the following primers were used:

Fw_VASH1_exCatCys 5'- GTGGGAGGTGGAAGTGAAAGAAGG-3';

Rv_VASH1_exCatCys 5'- GCCAAGCAGATGACTGCATATGG-3';

\begin{tabular}{|c|c|c|c|c|}
\hline \multicolumn{5}{|l|}{ Scheme } \\
\hline Cell line & Mutant & Clone & Vector & Transfected/Transduced \\
\hline \multirow[t]{6}{*}{ HEK293T } & Control & 1 & px330_empty & Transfected \\
\hline & Control & 2 & pxpr_001_empty /pLentiv2_Blast_empty & Transduced \\
\hline & $\triangle$ VASH1 & 1 & px330_VASH1_exCatCys & Transfected \\
\hline & $\triangle$ VASH1 & 2 & px330_VASH1_exCatCys & Transfected \\
\hline & $\triangle$ VASH $1 / 2$ & 1 & pxpr_001_VASH1_exCatCys/pLentiv2_Blast_VASH2_exCatCys & Transduced \\
\hline & $\triangle$ VASH $1 / 2$ & 2 & pxpr_001_VASH1_exCatCys/pLentiv2_Blast_VASH2_exCatCys & Transduced \\
\hline \multirow[t]{6}{*}{ CHL-1 } & Control & 1 & px330_empty & Transfected \\
\hline & Control & 2 & pxpr_001_empty/pLentiV2_Blast_empty & Transduced \\
\hline & $\triangle$ VASH1 & 1 & pxpr_001_VASH1_exCatCys & Transduced \\
\hline & $\triangle$ VASH1 & 2 & pxpr_001_VASH1_exCatCys & Transduced \\
\hline & $\triangle$ VASH $1 / 2$ & 1 & pxpr_001_VASH1_exCatCys/pLentiV2_Blast_VASH2_exCatCys & Transduced \\
\hline & $\Delta$ VASH $1 / 2$ & 2 & px330VASH1exCatCys/px330VASH2exCatCys & Transfected \\
\hline
\end{tabular}

Fw_VASH2_exCatCys 5' - TGCTATGGAGTACTGGCACTAACTCC-3';

Rv_VASH2_exCatCys 5'- GTCCAAGAAGCCAAGCATTGATTTGG-3'.

For Sanger Sequencing, the following primers were used:

Fw_VASH1_exCatCys_seq 5'-GGCCTCCTTCCTATCAGCAGTG-3' and 
Fw_VASH2_exCatCys_seq 5'- CTGCAAGGTGACTTCTCCCTTTTCC-3'

HEK293T and CHL-1 mutant cell lines: cells were either transfected or transduced as depicted in the scheme below. In transfections CMV-Blasticidin ${ }^{\text {res }}$ was co-transfected. HEK293T were transfected using Turbofectin (Origine \#TF81005) and CHL-1 cells were transfected using Lipofectamine 2000 (ThermoFisher scientific). For transductions standard lentivirus production protocols were used. 24 hours after transfection or 48 hours after transduction cells were selected using Blasticidin or Puromycin (Blasticidin HEK293T $50 \mu \mathrm{g} \mathrm{ml}^{-1}, \mathrm{CHL}-135$ $\mu \mathrm{g} \mathrm{ml} \mathrm{m}^{-1}$ Invivogen \#ant-bl-1; Puromycin HEK293T $3 \mathrm{\mu g} \mathrm{ml}^{-1}$ ) for 72 hours. Resistant cells were subcloned and gDNA was isolated as described before. CHL-1 cells were subjected to an additional round of subcloning. No obvious proliferation defects were observed in any of the generated knockout cell lines.

To identify clones bearing out-of-frame mutations or large deletions, leading to removal of the catalytic site cysteine. TIDE-analysis ${ }^{(23)}$ was performed and sanger reads were manually traced to identify mutant alleles.

TTL mutant cell lines showed no obvious cell fitness defect and were generated by cloning gRNA 5'-GTTCTCATCGCGTACCACGA-3' in px330. Hap1 cells were co-transfected with px330-TTL and CMV-Blasticidin res, selected clones were screened for TTL mutations by Western-blot using the TTL antibody previously mentioned. TUBA4A mutants were generated by cloning gRNA 5' GGTGGGCATCGACTCCTATG-3' into px330. Hap1 VASH1-2 deficient cells were transfected and selected with Blasticidin as mentioned before. Single colonies were picked from a plate and mutations were analysed using sanger sequencing of a PCR fragment obtained with TUBA4A_fw:

5'-CTATGCACCAGTCATCTCTCTGCAG-3' and

TUBA4A_rV:

5'- GGTCTCACCTT CAGCGATGGAAG-3'.

Sequencing primer 5'-GCCAACCAGATGGTAAAGTGTG ATC-3' was used to sequence the PCR product.

\section{Cloning of expression constructs}

SVBP-Flag, VASH1-Flag and VASH2-Flag were cloned from Hap1 cDNA, using Accuprime Hifi Taq polymerase (Thermofisher Scientific \#12346-086) using primers:

SVBP-FW:

5'-CAGTGAATTCGCCACCATGGATCCACCTGCACGTAAAG-3'

SVBP-RV:

5'-CATGGTCGACTCACTTATCGTCGTCATCCTTGTAATCTTCTCCAGGAGGCTGCATC-3'

VASH1-FW:

5'-CAGTGAATTCGCCACCATGCCAGGGGGGAAGAAGGTGGCTG-3'

VASH1-Rv: 
5'-CATGGTCGACTCACTTATCGTCGTCATCCTTGTAATCGACCCGG

ATCTGGTACCCGTT-3'

VASH2-FW:

5'-CAGTGAATTCGCCACCATGACCGGCTCCGCGGCCGACACTC-3'

VASH2-Rv:

5'-CATGGTCGACTCACTTATCGTCGTCATCCTTGTAATCAATTCGG

ATTTGATAGCCCAC-3'

Using standard cloning protocols, PCR products were cloned in pcDNA3.1(-) and VASH1-Flag in pMX-ires-Blast.

VASH1-Cys169Ala-Flag was obtained by fusion PCR of PCR products obtained with VASH1-Fw and

Rv_VASH1_C169A:

5' - GGATCACGGCTTCCAGGGCTTTGATTGGCAGGGCCTC-3'

FW_VASH1_C169A:

5'-GAGGCCCTGCCAATCAAAGCCCTGGAAGCCGTGATCC-3'

and VASH1-RV.

pcDNA3.1(-) containing VASH1 was used as a template. Using standard cloning protocols fusion PCR product was cloned in pcDNA3.1(-) VASH2-Cys93Ala-Flag was obtained by fusion PCR of PCR products obtained with VASH2-Fw and

Rv_VASH2_C93A:

5'-CCAGGATGACAGCTTCAAGGGCTTTGATAGGCAAGGACTC-3'

FW_VASH2_C93A:

5'-GAGTCCTTGCCTATCAAAGCCCTTGAAGCTGTCATCCTGG-3'

and VASH2-RV.

pcDNA3.1(-) containing VASH2 was used as a template. Using standard cloning protocols fusion PCR product was cloned in pcDNA3.1(-)

GFP-minimal substrates were obtained by PCR on eGFP template using primers:

FW_eGFP:

5'-TGATGATGCTAGCGCCACCATGGTGAGCAAGGGCGAGGAGCTGTTCAC-3'

$R v_{-}$TUBA1A/B:

5 '-agtagcggccgcTTAGTATTCCTCTCCTTCTTCCTCACCСTCTCCTTCAACA

GAATCCACACCAACCTCCTCATAATCCTTGTACAGCTCGTCCATGC-3'

Rv_TUBA3C:

5'-gagcggatcc TTAATACTCTTCACCCTCATCCTCTCCGTCAGCACTATCCTTGTAC AGCTCGTCCATGC-3'

Rv_TUBA4A:

5'- gagcggatcc TTATTCTTCTCCCTCATCCTCGTCCTCATAGGAGTCGATCTTGTA

CAGCTCGTCCATGC-3' 
Rv_TUBA8:

5'gagcggatccTTAAAATTCCTCCCCTTCATTTTCTTCTTCAAACGAATCCTTGTACAGCTCGTCCATGC-3'

GFP-TUBA1A/B mutants were obtained by using the eGFP-TUBA1A/B as a template and perform mutagenesis PCR using primers:

Fw_eGFP:

5’TGATGATGCTAGCGCCACCATGGTGAGCAAGGGCGAGGAGCTGTTCAC-3'

Rv_TUBA1B-F:

5'-GCTCGGATCCTTAGAATTCCTCTCCTTCTTCCTCAC-3'

Rv_TUBA1B-S:

5'-GCTCGGATCCTTAGGATTCCTCTCCTTCTTCCTCAC-3'

Rv_TUBA1B-A:

5'-GCTCGGATCCTTAGGCTTCCTCTCCTTCTTCCTCAC-3'

RV_TUBA1B-YG:

5'-GCTCGGATCCTTAACCGTATTCCTCTCCTTCTTCCTCAC-3'

RV_TUBA1B-GY:

5'-GCTCGGATCCTTAGTAACCTTCCTCTCCTTCTTCCTCAC-3'

Using standard cloning protocols purified PCR products were cloned in pCDNA3.1(-).

\section{Expression and purification of recombinant protein}

Codon-optimized synthetic genes encoding VASH1 and SVBP were cloned into pFastBac-derived vectors containing a C-terminal 2xstrepll-Flag tag (VASH1) or a C-terminal 6xHis-tag (SVBP) using Ligation Independent Cloning (LIC) as described in Luna-Vargas et al., ${ }^{(24)}$. The VASH1Cys169Ala mutant was created using the QuikChange cloning method (Agilent genomics). DNA Constructs were used for transposition into EMBACY Bacmid DNA according to Bac-toBac manufacturer procedures (ThermoFisher Scientific). Bacmid DNA was transfected into sf 9 insect cells and after 3 days baculovirus (PO) was harvested. Virus was amplified by addition of $0.5 \mathrm{ml}$ of PO virus stock to $25 \mathrm{ml}$ of $1 \times 10^{6} \mathrm{sf9}$ cells $\mathrm{ml}^{-1}$, grown in suspension. After 3 says, baculovirus was harvested ( $P 1$ stock) and these stocks were used to infect larger volumes of insect cells: $500 \mu$ l of SVBP-his P1 virus was used per $500 \mathrm{ml}$ of $1 \times 10^{6}$ insect cells to obtain SVBP-his protein. The VASH1-streplI-Flag/SVBP-his complex was produced upon co-infection of $500 \mathrm{ml} 1 \times 10^{6} \mathrm{sf9}$ cells $\mathrm{ml}^{-1}$ with $500 \mu \mathrm{l}$ of SVBP-his P1 virus and $500 \mu \mathrm{l}$ of VASH1-strepll-Flag $\mathrm{P} 1$ virus. Cells were harvested after 3 days by centrifugation at $1500 \times \mathrm{g}$ and cell pellet was stored at $-20^{\circ} \mathrm{C}$ until further use.

For protein purification, cells were resuspended in lysis buffer A ( 25 mM Tris-HCL pH 8.0, 200 $\mathrm{mM} \mathrm{NaCl}, 1 \mathrm{mM}$ TCEP) and lysed by sonication. After removal of cell debris and insoluble proteins by centrifugation at $50,000 \times \mathrm{g}$, the soluble fraction was used for affinity purification. SVBP-his was purified using Talon beads (ClonTech) and eluted in buffer A containing $200 \mathrm{mM}$ 
imidazole. SVBP-his was further purified by size exclusion chromatography on a Superdex S75 16/60 column (GE Healthcare) in buffer A. Fractions were pooled, concentrated and aliquots were stored at $-80^{\circ} \mathrm{C}$. The VASH1-strepll-Flag/SVBP-his complex was first purified using Talon beads as described above, followed by a second affinity purification using StrepTactin beads (IBA Lifesciences). Upon elution by $2.5 \mathrm{mM}$ Desthiobiotin in buffer A, fractions containing the complex were pooled, concentrated and aliquots were stored at $-80^{\circ} \mathrm{C}$. For part of the purified complex, tags were removed upon treatment with $3 \mathrm{C}$ protease after Streptactin purification, followed by size exclusion chromatography on a SEC650 column (Bio-Rad). Fractions containing the untagged-complex were pooled, concentrated and aliquots were stored at $-80^{\circ} \mathrm{C}$.

\section{Size Exclusion Small Angle X-ray Scattering (SAXS) data collection}

SAXS data were collected at Beamline BM29 at ESRF (Grenoble). About $40 \mu \mathrm{l}$ of either SVBP or VASH1-SVBP complex were loaded onto a Superose- 6 column at a concentration 3-5 $\mathrm{mg} \mathrm{ml}^{-1}$. The flow rate was adjusted to $1 \mathrm{ml} \mathrm{min} \mathrm{m}^{-1}$ and the scattering profile of the eluate was collected in 1100 successive frames of $1 \mathrm{~s}$; the full absorption spectrum was also measured. The protein elution peak was determined from that data and the scattering intensities of the corresponding frames were averaged. The scattering from the solvent was subtracted, according to standard procedures.

\section{SAXS data analysis}

The data were analyzed using the ATSAS and ScÅtter software packages to obtain model independent parameters such as the radius of gyration $\left(R_{g}\right)$, the maximal distance $\left(D_{\max }\right)$ and the volume of correlation $\left(V_{c}\right)^{(25,26)}$. The molecular mass was calculated using the Porod volume, and the $Q_{R}$ method $^{(27,28)}$. The ambiguity of the two datasets was measured using the program AMBIMETER ${ }^{(29)}$. The SVBP dataset is characterized by higher ambiguity; in the complex with VASH1 the ambiguity score decreases suggesting that the $3 \mathrm{D}$ reconstruction is potentially unique, and indicating the stabilizing role of SVBP on the VASH1-SVBP complex. To create an ab initio model for VASH1-SVBP complex, data range was selected based on Shannon sampling according to the program SHANUM ${ }^{(30)}$. DAMMIN was used to create an ab initio model from 16 independent runs. Averaging was performed using SUPCOMB and DAMAVER program ${ }^{(31)}$. In addition, a multiphase volumetric analysis was performed using MONSA and the two datasets of SVBP and VASH1-SVBP. An averaged and aligned MONSA model was calculated from 20 independent MONSA runs, indicating a plausible model for the SVBP and VASH1 placement.

\section{In vitro detyrosination assays}

In vitro detyrosination assay: $200 \mathrm{nM}$ of Vasohibin:SVBP, Vasohibin-C169A or SVBP was incubated with $50 \mathrm{nM}$ of purified $\alpha / \beta$-tubulin dimers (TeBu-Bio 027CS-H001-B) in buffer containing $50 \mathrm{mM}$ Tris- $\mathrm{HCl} \mathrm{pH} \mathrm{8.0;} \mathrm{10 \%} \mathrm{glycerol;} 1 \mathrm{mM} \mathrm{DTT;} 1 \mathrm{mM} \mathrm{PMSF}$ and was incubated for 2 hours at $37^{\circ} \mathrm{C}$. Samples were mixed with sample buffer (as mentioned before) and subjected to immunoblotting. To determine $\mathrm{K}_{\mathrm{m}}, 200 \mathrm{pM}$ of enzyme was incubate with a 1:1 dilution series of HeLa cells purified $\alpha / \beta$-tubulin dimers assay conditions as described above. The reactions were 
incubated for 8 hours at $37^{\circ} \mathrm{C}$ and dot-blotted on nitrocellulose (GE Healthcare \#10600001). The blots were digitally imaged; as the dynamic range of the tubulin intensities was too broad to assay in a single blot, the assay was performed over two blots: one with high and one with low concentrations of HeLa cells purified $\alpha / \beta$-tubulin dimers. All experiments were done in triplicate. To estimate the intensity of each dot, a square block was isolated and the values of the pixels were summed; the background was measured by placing a square of identical size in randomly chosen areas of the blot. To bring both the intensities of dots on both blots in scale, a scaling factor was calculated from the background of the two blots. The $\mathrm{K}_{\mathrm{M}}$ was then estimated using standard methods in GraphPad/Prism.

The assay to determine the difference in detyrosination activity of SVBP: VASH1 towards polymerized and non-polymerized $\alpha / \beta$-tubulin was performed on in vitro generated microtubules. To polymerize, $3 \mathrm{mg} \mathrm{m}^{-1} \alpha / \beta$-tubulin was incubated in the presence of $1 \mathrm{mM}$ GTP, $10 \%$ glycerol and $10 \mu \mathrm{M}$ paclitaxel. The reaction was incubated at $37^{\circ} \mathrm{C}$ for $90 \mathrm{~min}$ and polymerized MTs were diluted 3-fold with General Tubulin Buffer, supplemented with $10 \mu \mathrm{M}$ paclitaxel, before added to an in vitro reaction as described above, supplemented with $20 \mu \mathrm{M}$ paclitaxel. The same reaction was performed without the addition of GTP to prevent efficient polymerization. To quantify the difference in the detyrosination rate of VASH1:SVBP, the detyrosinated and the total tubulin levels were measured by immunoblot analysis. The bands were quantified as described above. To account for differences in the efficiency of blotting between experiments; a linear scale was applied between equivalent datasets from different experiments; scale factors were calculated by linear regression to minimise the least squared diffenence between all points in all three equivalent datasets. The average for each dataset (deY non-polymerized, total non-polymerized, total polymerized) and the standard error were calculated, and the ratio of deY vs total tubulin were calculated, together with the standard error of ratio, to calculate the initial enzymatic rate of detyrosination. A linear model was fit to estimate the rates of hydrolysis for the two reactions.

\section{Proteomics}

Mock- and VASH1-treated tubulin standard was separated on gel, tubulin bands ( $~ 50 \mathrm{kDa})$ were excised, reduced with $6.5 \mathrm{mM}$ DTT, alkylated with $54 \mathrm{mM}$ iodoacetamide and in-gel digested with Lys-N (10 ng $\left.\mu^{-1}\right)$ overnight at $37^{\circ} \mathrm{C}$. Extracted peptides were vacuum dried, reconstituted in 10\% formic acid and analyzed by nanoLC-MS/MS on an Orbitrap Fusion Tribrid mass spectrometer equipped with a Proxeon nLC1000 system (Thermo Scientific). Peptides were loaded directly on the analytical column, separated using a linear 45-min gradient and further settings were as described previously ${ }^{(32)}$. Raw data files were processed with Proteome Discoverer (version 1.4.1.14, Thermo Scientific), searching against the human swissprot database (release 2016_01, 20.187 entries), using Mascot (version 2.5, Matrix Science, UK). Carbamidomethylation of cysteines and oxidation of methionine were set as fixed and variable modifications, respectively. Semi-Lys-N was specified as enzyme and up to two miscleavages 
were allowed. Data filtering was performed using percolator, resulting in $1 \%$ false discovery rate (FDR) at peptide level. Additional filters were search engine rank 1 peptides and ion score $>20$. To assess the relative composition of tyrosinated and non-tyrosinated tubulin upon VASH1 treatment, extracted ion chromatograms of the C-terminal peptide with and without tyrosine were generated in Thermo Xcalibur Qual Browser software (version 3.0.63).
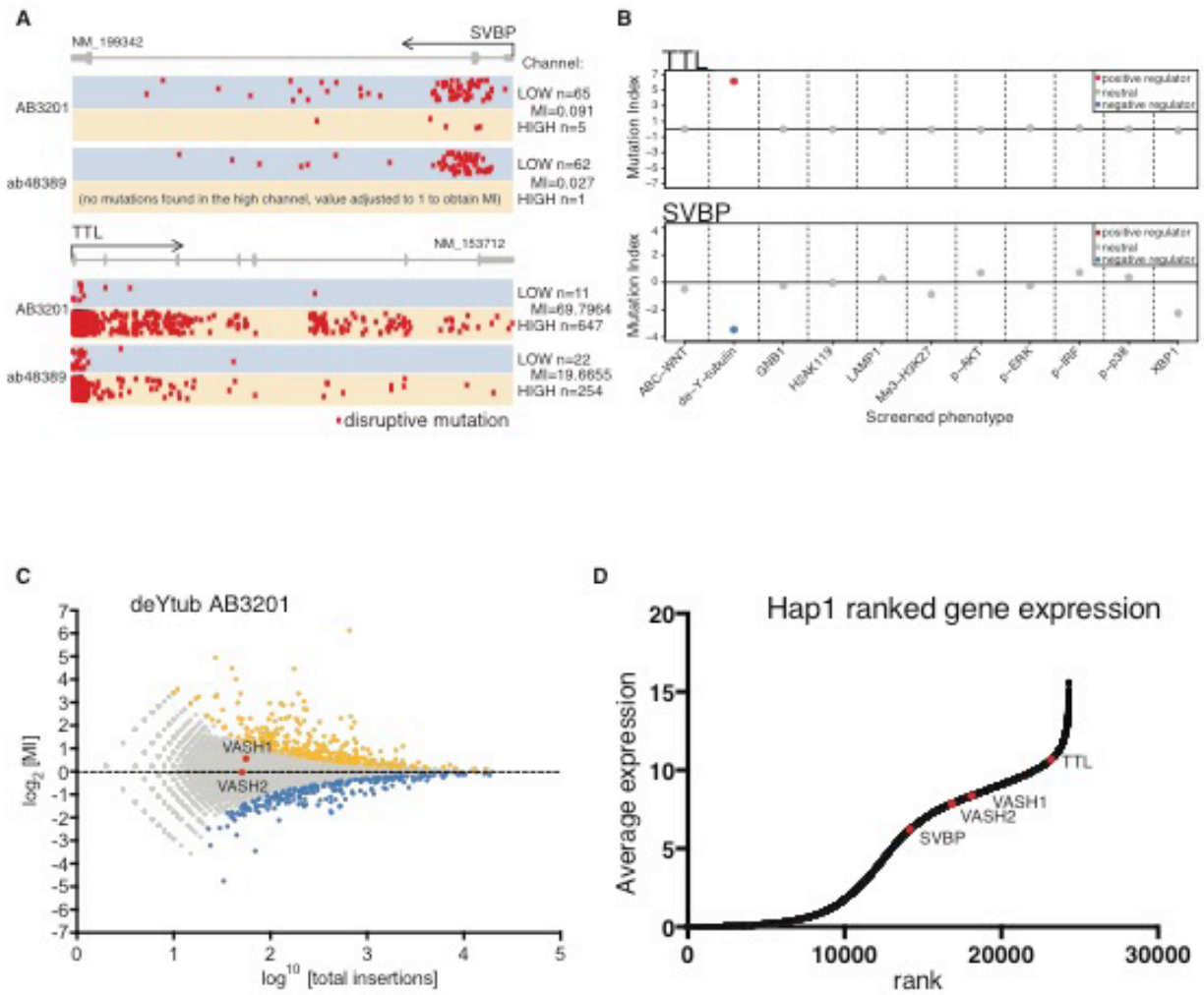

Fig. S1. Mutations of Vasohibins and SVBP in different genetic screens and their expression in Hap1 cells. (A) Individual mutations in SVBP and TTL obtained in the screen represented in Fig. 1B and a selection using an independent antibody. Selection for cells with low amounts of detyrosinated $\alpha$-tubulin are enriched for SVBP mutations (plotted on a yellow background), whereas selection for cells in the "high" channel were found to be enriched for TTL mutations (plotted on a grey background). Disruptive sense-orientation gene-trap integrations are counted and depicted. (B) The MI-values for SVBP and TTL are plotted for 10 other previously-published screens, indicating that they are uniquely identified as regulators of tubulin detyrosination. (C) Screen for genetic regulators of detyrosinated $\alpha$-tubulin as shown in Fig $1 \mathrm{C}$, showing that neither VASH1 nor VASH2 (highlighted) was identified as significant regulator of $\alpha$-tubulin detyrosination. (D) Ranked gene expression based on RNAseq data of wild-type Hap1 cells, available in Brockmann et al (9). Average expression was calculated and non-coding genes were removed. VASH1, VASH2, SVBP and TTL were highlighted. 


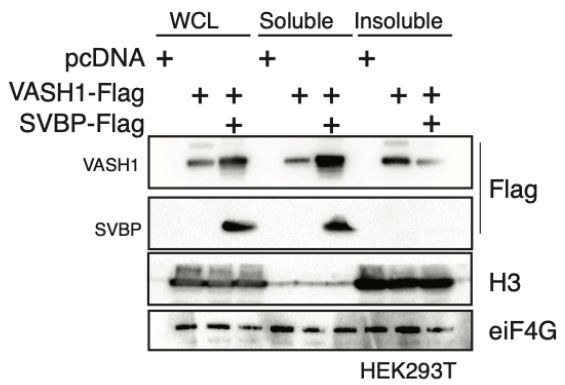

Fig. S2. SVBP co-transfection results in increased levels of soluble VASH1. HEK293T cells were transfected with plasmids encoding VASH1-Flag, SVBP-Flag or a combination of both. From these cells, a Whole Cell Lysate (WCL), a soluble fraction and an insoluble fraction were obtained. These fractions were subjected to immunoblot analysis showing that cells that co-express SVBP-Flag with VASH1-Flag have higher levels of soluble VASH1-Flag, and SVBP-Flag itself is detected in the soluble fraction. 

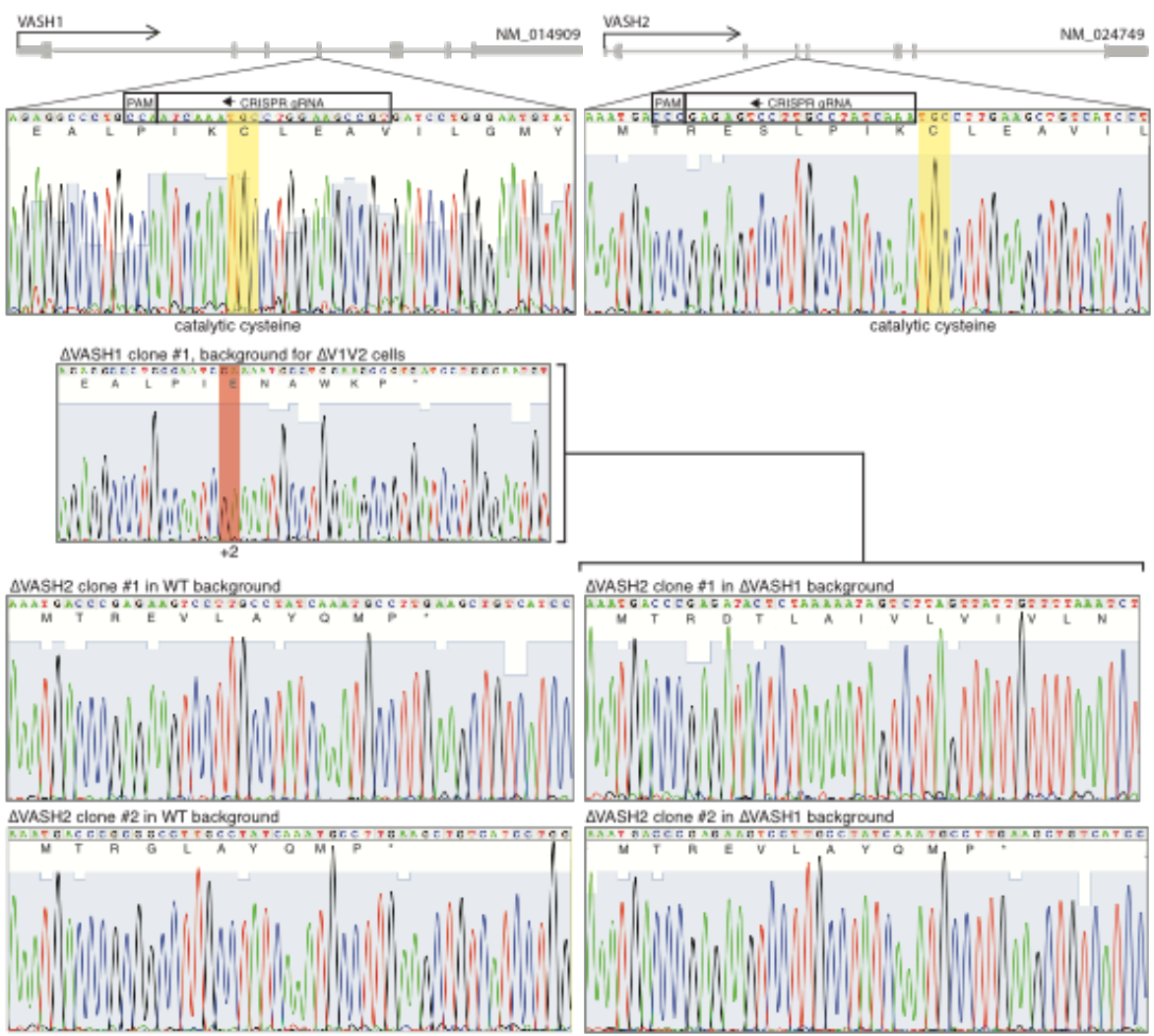

$\triangle V A S H 2$ clone 12 in $\triangle V A S H 1$ background
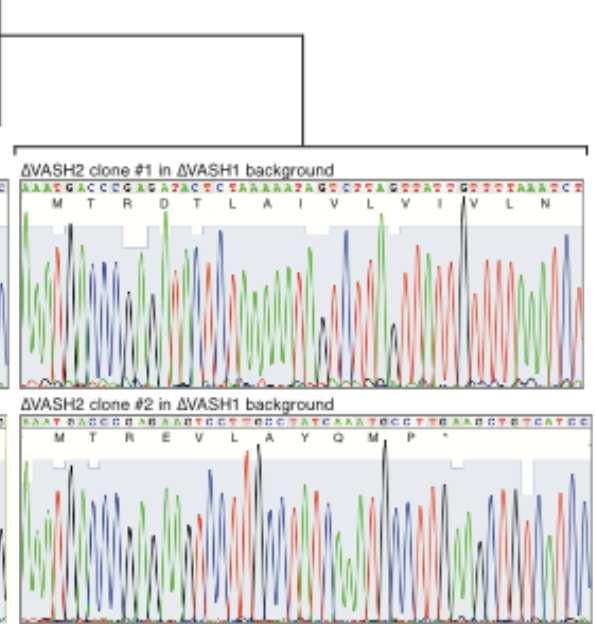

$\triangle$ VASH2 clone $A 3$ in WT background
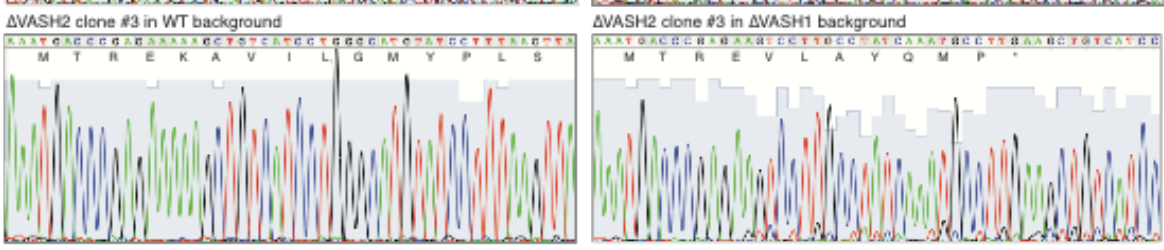

\begin{tabular}{|c|c|c|c|c|}
\hline background & WT & & $\triangle$ VASH1 & \\
\hline WT & ACCCGAGAGTCCTTGCCTATCAAATECCTT & 1ndel & ACCCGAGAGTCCTRGCCTATCAAATVCCTT & indel \\
\hline$\Delta V 2$ & ACCCGAGAAGTCCTTGCCTATCAAATECCT & +1 & ACCCGAGATACTCYAAAAATAGTCTTAGTT & +60 \\
\hline$\Delta \mathrm{V} 2$ & ACCOGCGG: \& CCTTGCCTATCAAATCCCTT & -2 & ACCCGAGAAGTCCPTOCCZATCAAATCCCT & +1 \\
\hline$\Delta V_{2}$ & ACCCCAAGARA: ::::::::::1: & -21 & ACCCGAGAAGTCCRTOCCPATCAAATCCCT & +1 \\
\hline
\end{tabular}

Fig. S3. Generation of Hap1 cells deficient for VASH1, VASH2 or VASH1-2 using CRISPR/CAS9. Plasmids encoding Cas9 as well as a gRNA predicted to cause a mutation at just upstream of the catalytic cysteine critical for Vasohibindependent tubulin detyrosinating activity were transfected in Hap1 cells. Sanger sequencing of PCR products of the affected locus of clonal cell lines show genomic mutations at the respective loci and the altered amino acid sequences are indicated. 


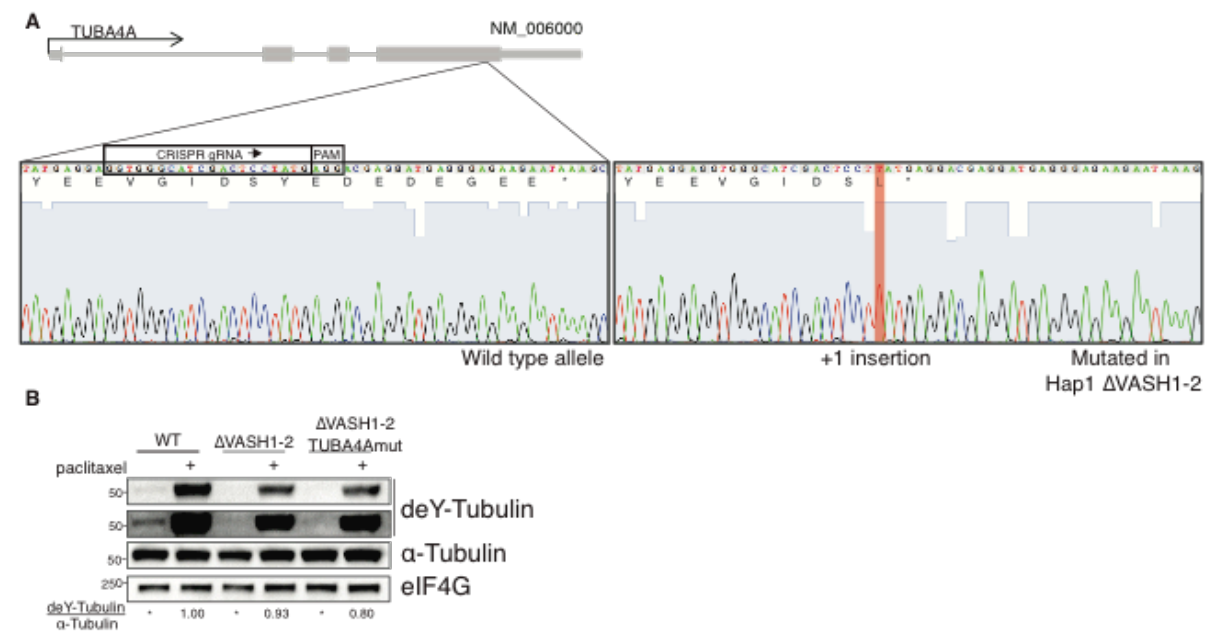

Fig. S4. TUBA4A does not explain the remaining levels of tubulin detyrosination present in VASH1-2 deficient cells. (A) gRNA targeting the 3'end of TUBA4A was used to mutate the c-terminal tail of TUBA4A, in cells deficient for VASH12, resulting in an early stop-codon, preventing the expression of the "detyrosination epitope". (B) WT, VASH1-2 deficient cells and VASH1-2 deficient cells with the mutation shown in (A). These cells were treated with paclitaxel, showing a similar increase in detyrosination in both the VASH1-2 deficient cells as the cells lacking VASH1-2 in combination with the C-terminal tail of TUBA4A, excluding a significant role for TUBA4A in the observed increase in paclitaxel-induced detyrosination. 

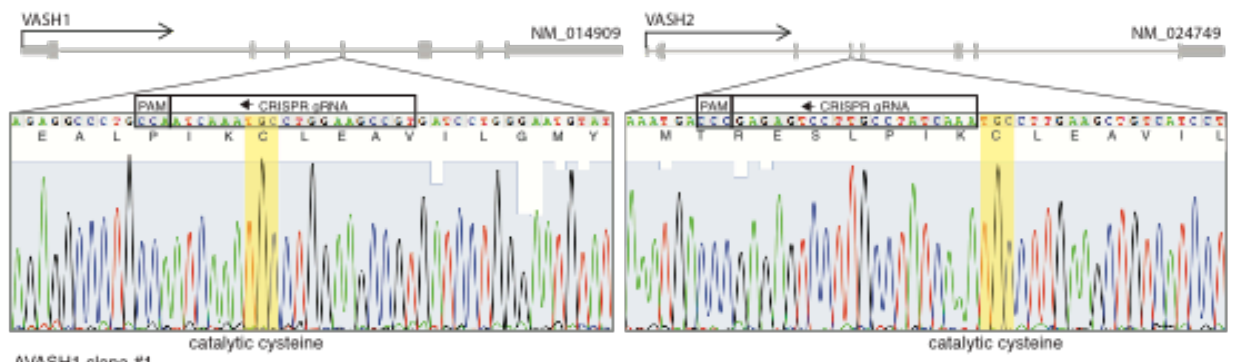

$\triangle$ VASH1 clone \#1

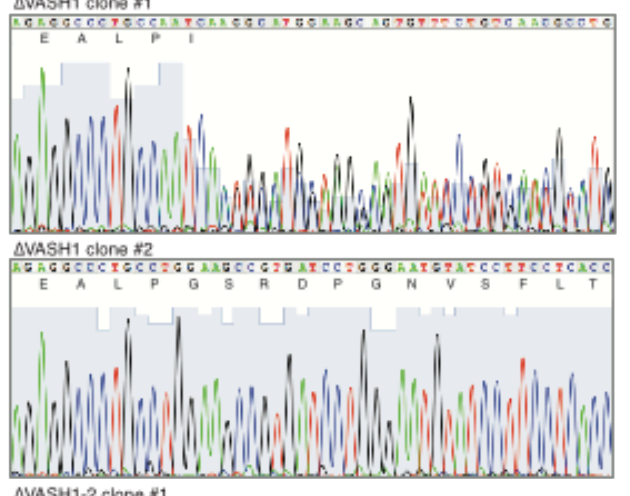

$\triangle$ VASH1-2 clane $* 1$



Decormaluted alleles

\begin{tabular}{|c|c|c|c|c|}
\hline & VASH1 & & VASH2 & \\
\hline WT & СССТGCCAATCAAATCCCTGGAAGCCGTC & indel & CAAATCACCCCAGAATCCFTOCCYATCAAATCCCTT & indel 1 \\
\hline$\Delta V 1$ & 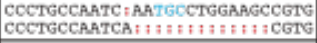 & $\begin{array}{l}-1 \\
-13\end{array}$ & - & - \\
\hline$\Delta \mathrm{V}_{1}$ & CCCTGCC:::::::1:::TGGAAGCCGTG & -11 & - & - \\
\hline$\Delta V 1 \Delta V_{2} \# 1$ & 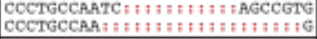 & $\begin{array}{l}-11 \\
-19\end{array}$ &  & $\begin{array}{l}+1 \\
-4\end{array}$ \\
\hline$\Delta V 1 \Delta V 2 \# 2$ & 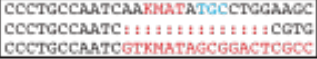 & $\begin{array}{l}+4 \\
-14 \\
+124\end{array}$ & 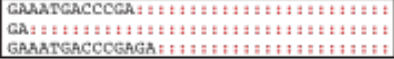 & $\begin{array}{l}-89 \\
-128 \\
-130\end{array}$ \\
\hline
\end{tabular}

Fig. S5. Generation of HEK293 cells deficient for VASH1, VASH2 or VASH1-2 using CRISPR/CAS9. CRISPR/Cas9 variants targeting the exon encoding the catalytic cysteine critical for Vasohibin-dependent tubulin detyrosination activity were used to obtain the indicated mutations. For a detailed overview of applied approach per clone see methods section. 
Vasohibin mutants in CHL-1 as used in figure $2 \mathrm{~B}$
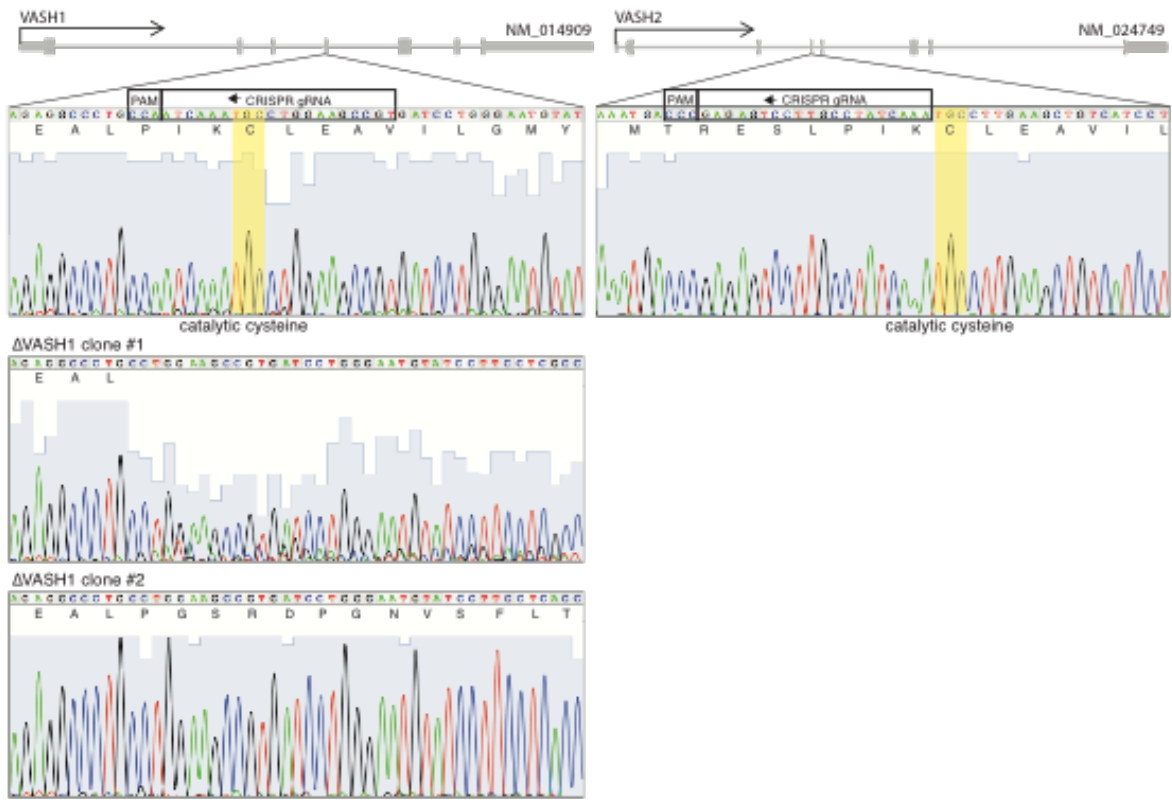

DVASH1-2 clane $\# 1$
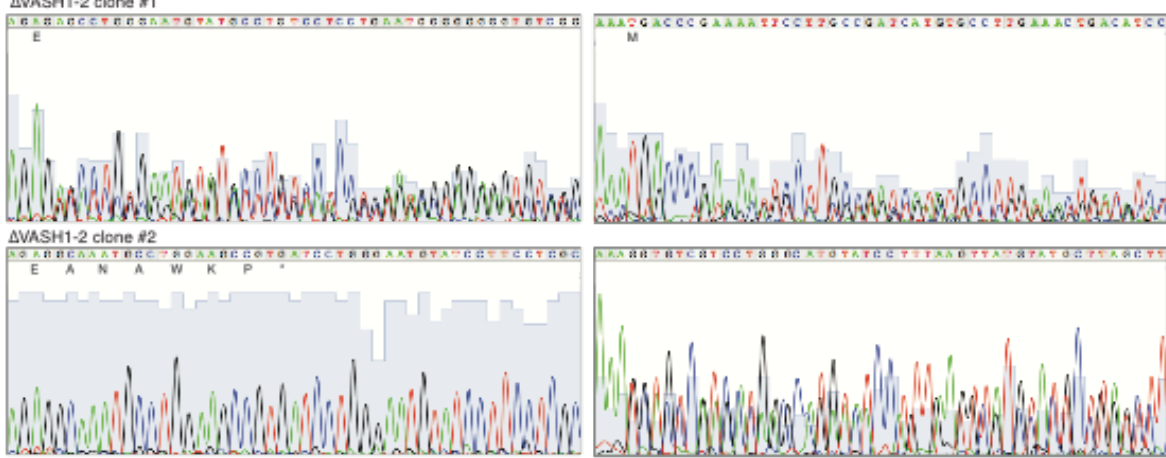

\section{Linerese}



Deconwoluted alleles

\begin{tabular}{|c|c|c|c|c|c|}
\hline & & \multicolumn{2}{|l|}{ VASB 1} & \multicolumn{2}{|l|}{ VASH2 } \\
\hline WT & & AGECCCTVCCAATCAAATECCTEGAAGCCGTG & indel 1 & AGAAATGACCCGAGAGTCCTTGCCTATCAAATGCCTT & indel \\
\hline$\Delta \mathrm{V}_{1}$ & \#1 & 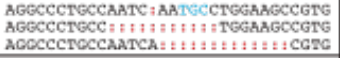 & $\begin{array}{l}-1 \\
-11 \\
-13\end{array}$ & - & - \\
\hline$\Delta \mathrm{V}_{1}$ & *2 & 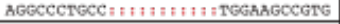 & -11 & - & - \\
\hline$\Delta V_{1} \Delta V_{2}$ & *1 & 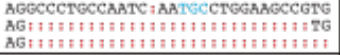 & $\begin{array}{l}-1 \\
-28 \\
-30\end{array}$ & 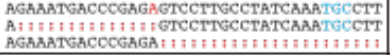 & $\begin{array}{l}+1 \\
-14 \\
-26\end{array}$ \\
\hline$\Delta V_{1} \Delta V_{2} \#$ & & ACOC: : : : : : : : : : : : AAATOCCTOCAACCCGTC & -10 & 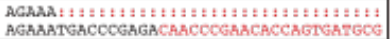 & $\begin{array}{l}-35 \\
+56\end{array}$ \\
\hline
\end{tabular}

Fig. S6. Generation of CHL-1 cells deficient for VASH1, VASH2 or VASH1-2 using CRISPR/CAS9. CRISPR/Cas9 variants targeting the exon encoding the catalytic cysteine critical for Vasohibin-dependent tubulin detyrosination activity were used to obtain the indicated mutations. For a detailed overview of applied strategy per clone see methods section. 
A



B

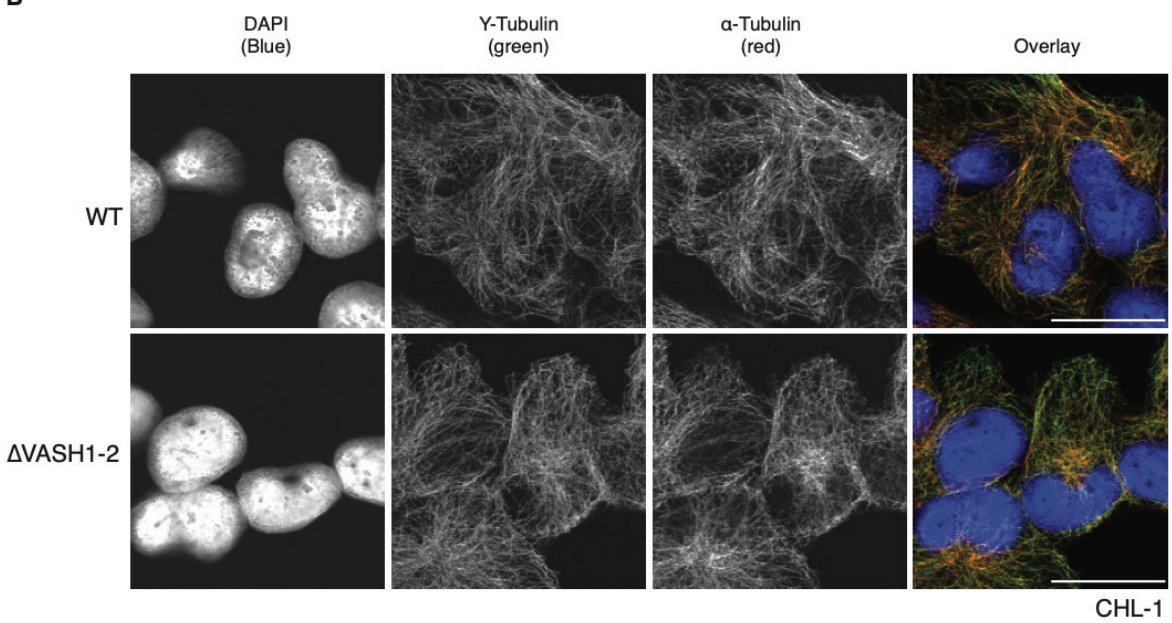

Fig. S7. Tyrosinated $\square$-tubulin levels are modestly affected by a decrease in detyrosination levels. (A) Wild-type Hap1 (same lysates as used in figure $2 \mathrm{C}$ ) and CHL-1 cells as well as Hap1 cells and CHL-1 deficient for VASH1-2 were treated with paclitaxel and subjected to immunoblot analysis using an antibody specifically recognizing the tyrosinated form of $\alpha$-tubulin. In both Hap1 as CHL-1 cells only a moderate increase is in tubulin tyrosination is detected in vasohibin deficient cells. (B) Immunofluorescence of WT CHL-1 cells showing a moderate increase in tubulin tyrosination levels in cells deficient for vasohibins.
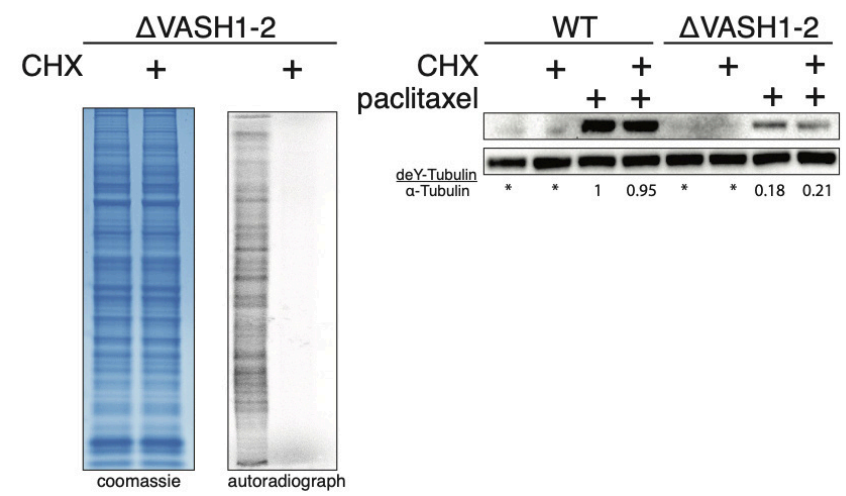

Fig. S8. Protein synthesis is not required for the generation of detyrosinated tubulin in vasohibin deficient cells. WT and VASH1-2 deficient cells were treated with cycloheximide (CHX) (160 ug ml-1), paclitaxel or a combination of both and subjected to immunoblot analysis. Short (1 hour) co-treatment of $\mathrm{CHX}$ with paclitaxel does not affect the levels of detyrosinated tubulin, illustrating that tubulin detyrosination in Vasohibin-deficient cells takes place on the pre-existing pool of tubulin molecules. 


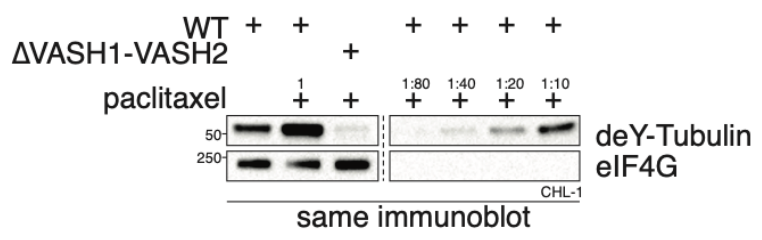

Fig. S9. Comparative Western-Blot analysis of paclitaxel treated WT and Vasohibin-deficient CHL-1 cells. To estimate the difference in total detyrosinated $\alpha$-tubulin levels in WT cells compared to Vasohibin deficient cells, a diluted sample of VASH1-WT cells treated with paclitaxel was compared to a paclitaxel treated lysate obtained from Vasohibin deficient cells. Immunoblot analysis was carried out using antibodies specific for detyrosinated $\alpha$-tubulin and elF4G.

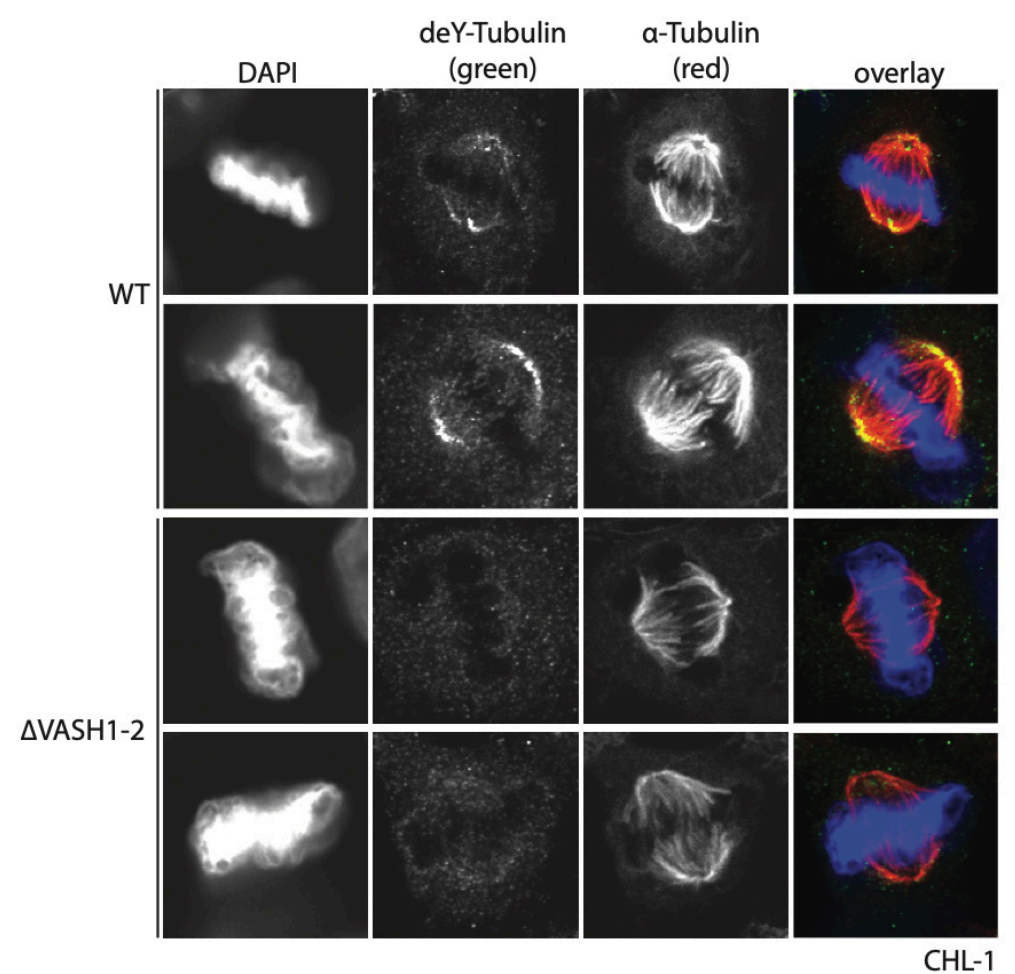

Fig. S10. Spindle microtubules are subjected to detyrosination by Vasohibins. Wild type and Vasohibin deficient CHL1 cells were treated with MG132 to enrich for mitotic spindles, followed by fixation and immunostaining for $\alpha$-tubulin and detyrosinated tubulin. In WT cells, co-localization of the detyrosinated signal with the tubulin signal is observed, which is absent in VASH1-2 knock out cells. 
A

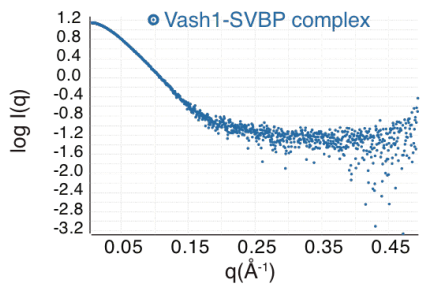

c

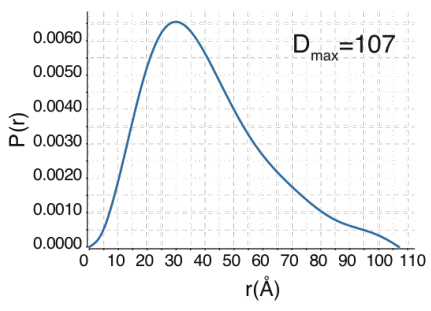

F

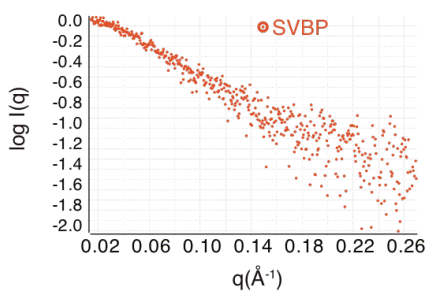

H

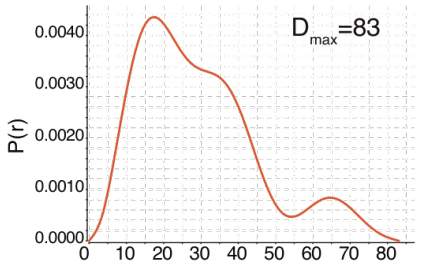

B

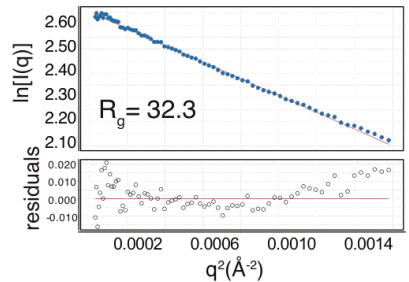

D

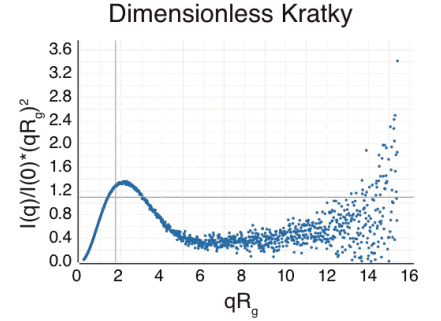

G

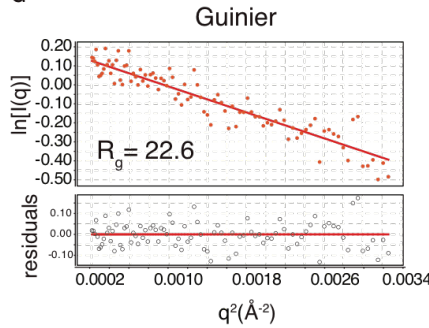

I

\section{Dimensionless Kratky}

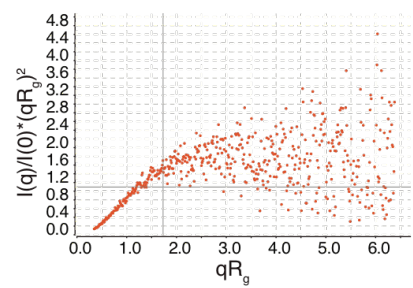

$\mathbf{J}$

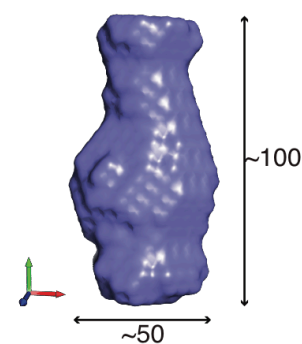

5

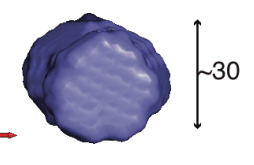

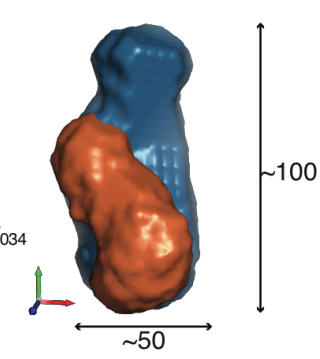

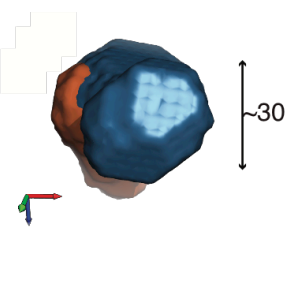

Fig. S11. SAXS analysis of the VASH1-SVBP complex and SVBP. (A) Scattering curve of VASH1:SVBP complex. (B) Guinier analysis of VASH1:SVBP complex, indicating that the sample is monodisperse. (C) The interatomic distance probability distribution $\mathrm{P}(\mathrm{r})$ of VASH1:SVBP suggesting that the shape of the complex is not spherical but elongated. (D) Dimensionless Kratky plot of VASH1:SVBP complex. The observed shift to the right of the peak indicated by the two grey axes, suggests that the shape of VASH1:SVBP is not spherical. (E) Averaged and aligned DAMMIN ab initio model of VASH1:SVBP. Rotation is performed $90^{\circ}$ along the $x$-axis. Average was determined from 16 independent DAMMIN runs. (F) Scattering curve of SVBP. (G) Guinier analysis of SVBP protein. (H) The interatomic distance probability distribution $P(r)$ of SVBP indicating the non-spherical shape of the protein. (I) Dimensionless Kratky plot of SVBP. (J) Averaged and aligned MONSA model of VASH1:SVBP protein complex. Average was determined from 20 independent MONSA runs. Rotation is performed $90^{\circ}$ along the $x$-axis. 


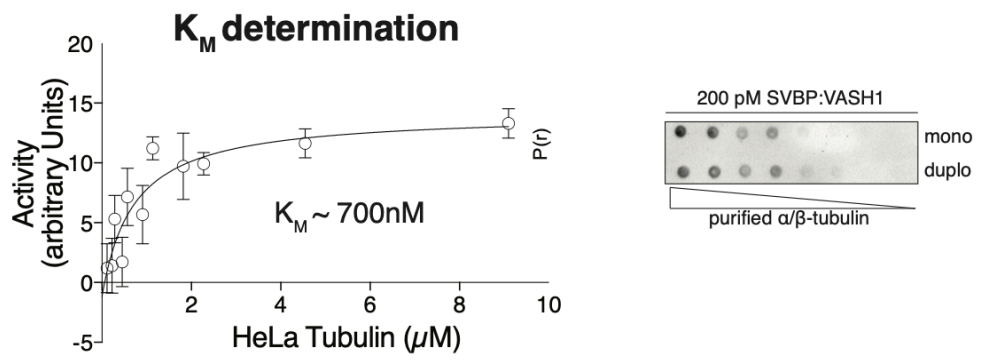

Fig. S12. Determination of the $K_{M}$ of SVBP:VASH1 complex towards purified $\alpha / \beta$-tubulin. (A) Purified SVBP-VASH1 was incubated with $\alpha / \beta$-tubulin purified from HeLa cells and analyzed by quantitative dot-blot analysis using antibodies directed against detyrosinated $\alpha$-tubulin to determine the $K_{M}$ value. (B) Example of a dot-blot used for $K_{M}$-analysis based on the in vitro detyrosination assay presented in (A). A 1:1 dilution of $\alpha$-tubulin was incubated with a fixed amount (200 pM) of SVBP:VASH1 complex for 8 hours. Reaction was spot-blotted on a membrane and subjected to immunoblotting using an antibody recognizing detyrosinated tubulin. Dots were subjected to quantification using FIJI software.

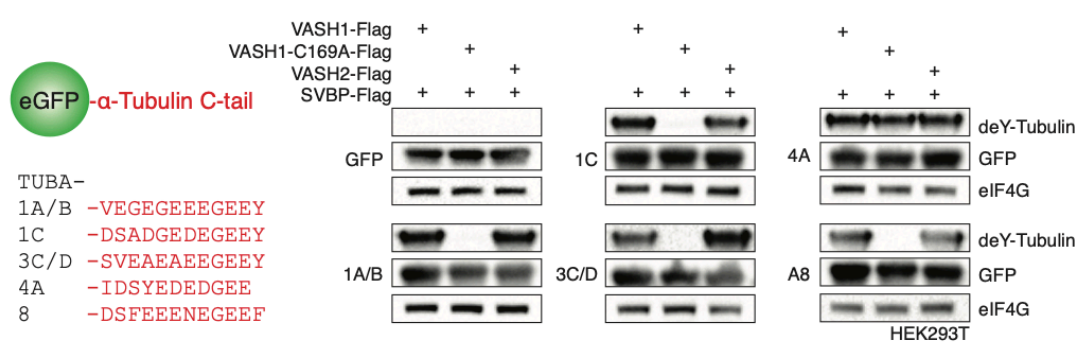

Fig. S13. All tubulin C-terminal tails encoding an aromatic c-terminus can be subjected to enzymatic processing by Vasohibins. Co-expression of vectors encoding VASH1, VASH1-C169A and VASH2 and minimal substrates consisting of the C-terminal tails of all $\alpha$-tubulin isoforms attached to enhanced Green Fluorescent Protein (eGFP) in HEK293T cells. Detyrosination activity was determined by immunoblot analysis using specific antibodies. elF4G was used as a loading control. 


\section{REFERENCES}

1. S. Gadadhar, S. Bodakuntla, K. Natarajan, C. Janke, The tubulin code at a glance. J. Cell. Sci. 130, 1347-1353 (2017).

2. H. Murofushi, Purification and characterization of tubulin-tyrosine ligase from porcine brain. J. Biochem. 87 979-984 (1980).

3. M. E. Hallak, J. A. Rodriguez, H. S. Barra, R. Caputto, Release of tyrosine from tyrosinated tubulin. Some common factors that affect this process and the assembly of tubulin. FEBS Lett. 73, 147-150 (1977).

4. P. Robison et al., Detyrosinated microtubules buckle and bear load in contracting cardiomyocytes. Science. $\mathbf{3 5 2}$ aaf0659-aaf0659 (2016).

5. J. R. Yoon et al., Local anesthetics inhibit kinesin motility and microtentacle protrusions in human epithelial and breast tumor cells. Breast Cancer Res. Treat. 129, 691-701 (2011).

6. M. Barisic et al., Mitosis. Microtubule detyrosination guides chromosomes during mitosis. Science. 348, 799-803 (2015).

7. C. P. Garnham, A. Roll-Mecak, The chemical complexity of cellular microtubules: tubulin post-translational modification enzymes and their roles in tuning microtubule functions. Cytoskeleton (Hoboken). 69, 442-463 (2012).

8. J. E. Carette et al., Haploid genetic screens in human cells identify host factors used by pathogens. Science. $\mathbf{3 2 6}$ 1231-1235 (2009).

9. M. Brockmann et al., Genetic wiring maps of single-cell protein states reveal an off-switch for GPCR signalling Nature. 546, 307-311 (2017).

10. K. Jiang et al., Microtubule minus-end stabilization by polymerization-driven CAMSAP deposition. Dev. Cell. 28 295-309 (2014).

11. J. T. Fassett et al., AMPK attenuates microtubule proliferation in cardiac hypertrophy. Am. J. Physiol. Heart Circ. Physiol. 304, H749-58 (2013).

12. Y. Suzuki et al., Isolation of a small vasohibin-binding protein (SVBP) and its role in vasohibin secretion. J. Cell. Sci. 123, 3094-3101 (2010).

13. L. Sanchez-Pulido, C. P. Ponting, Vasohibins: new transglutaminase-like cysteine proteases possessing a noncanonical Cys-His-Ser catalytic triad. Bioinformatics. 32, 1441-1445 (2016).

14. M. Saito, Y. Suzuki, S. Yano, T. Miyazaki, Y. Sato, Proteolytic inactivation of anti-angiogenic vasohibin-1 by cancer cells. J. Biochem. 160, 227-232 (2016).

15. X. Xue et al., Vasohibin 2 is transcriptionally activated and promotes angiogenesis in hepatocellular carcinoma. Oncogene. 32, 1724-1734 (2013).

16. J. C. Bulinski, J. E. Richards, G. Piperno, Posttranslational modifications of alpha tubulin: detyrosination and acetylation differentiate populations of interphase microtubules in cultured cells. J. Cell Biol. 106, 1213-1220 (1988).

17. A. E. Prota et al., Structural basis of tubulin tyrosination by tubulin tyrosine ligase. J. Cell Biol. 200, 259-270 (2013).

18. C. Aillaud et al., Evidence for new C-terminally truncated variants of $\alpha$-and $\beta$-tubulins. Mol. Biol. Cell. 27, 640-653 (2016).

19. K. Yoshinaga et al., Roles of intrinsic angiogenesis inhibitor, vasohibin, in cervical carcinomas. Cancer Sci. 102 446-451 (2011).

20. H. Kimura et al., Distinctive localization and opposed roles of vasohibin-1 and vasohibin-2 in the regulation of angiogenesis. Blood. 113, 4810-4818 (2009).

21. J. Kern, M. Steurer, G. Gastl, E. Gunsilius, G. Untergasser, Vasohibin inhibits angiogenic sprouting in vitro and supports vascular maturation processes in vivo. BMC Cancer. 9, 284-11 (2009).

22. A. E. Carpenter et al., CellProfiler: image analysis software for identifying and quantifying cell phenotypes. Genome Biol. 7, R100-11 (2006).

23. E. K. Brinkman, T. Chen, M. Amendola, B. van Steensel, Easy quantitative assessment of genome editing by sequence trace decomposition. Nucleic Acids Research. 42, e168-e168 (2014).

24. M. P. A. Luna-Vargas et al., Enabling high-throughput ligation-independent cloning and protein expression for the family of ubiquitin specific proteases. Journal of Structural Biology. 175, 113-119 (2011).

25. D. Franke et al., ATSAS 2.8: a comprehensive data analysis suite for small-angle scattering from macromolecular solutions. J App/ Crystallogr. 50 (2017), doi:10.1107/S1600576717007786.

26. J. C. Grigg, A. Ke, Structures of Large RNAs and RNA-Protein Complexes: Toward Structure Determination of 
Riboswitches. Meth. Enzymol. 558, 213-232 (2015).

27. R. P. Rambo, J. A. Tainer, Accurate assessment of mass, models and resolution by small-angle scattering. Nature 496, 477-481 (2013).

28. R. P. Rambo, J. A. Tainer, Characterizing flexible and intrinsically unstructured biological macromolecules by SAS using the Porod-Debye law. Biopolymers. 95, 559-571 (2011).

29. M. V. Petoukhov, D. I. Svergun, Ambiguity assessment of small-angle scattering curves from monodisperse systems. Acta Crystallogr. D Biol. Crystallogr. 71, 1051-1058 (2015).

30. P. V. Konarev, D. I. Svergun, A posterioridetermination of the useful data range for small-angle scattering experiments on dilute monodisperse systems. IUCrJ. 2, 352-360 (2015).

31. M. B. Kozin, D. I. Svergun, Automated matching of high- and low-resolution structural models. J App/ Crystallogr. 34, 33-41 (2001).

32. N. Ameziane et al., A novel Fanconi anaemia subtype associated with a dominant-negative mutation in RAD51. Nat Commun. 6, 8829-11 (2015). 



\section{Chapter 3}

\section{Crystal structure of the VASH1/ SVBP complex, a tubulin tyrosine carboxypeptidase}

Athanassios Adamopoulos ${ }^{1}$, Lisa Landskron ${ }^{1}$, Tatjana Heidebrect ${ }^{1}$, Foteini Tsakou ${ }^{1}$, Onno B. Bleijerveld ${ }^{2}$, Maarten Altelaar ${ }^{2,3}$, Joppe Nieuwenhuis ${ }^{1}$, Patrick Celie ${ }^{1}$, Thijn R Brummelkamp ${ }^{1,4,5,6}$ and Anastassis Perrakis ${ }^{1}$

\footnotetext{
${ }^{1}$ Division of Biochemistry, The Netherlands Cancer Institute, Amsterdam, The Netherlands ${ }^{2}$ The Netherlands Cancer Institute, Proteomics Facility, Amsterdam, The Netherlands

${ }^{3}$ Biomolecular Mass Spectrometry and Proteomics, Bijvoet Center for Biomolecular Research and Utrecht Institute for Pharmaceutical Sciences, Utrecht University, The Netherlands

${ }^{4}$ CeMM Research Center for Molecular Medicine of the Austrian Academy of Sciences, 1090 Vienna, Austria.

${ }^{5}$ Oncode Institute, Division of Biochemistry, Netherlands Cancer Institute, Plesmanlaan 121, 1066 CX, Amsterdam, Netherlands

${ }^{6}$ Cancer Genomics Center (CGC.nl), Plesmanlaan 121, 1066 CX, Amsterdam, Netherlands

Correspondence should be addressed to a.perrakis@nki.nl
}

\section{Based on:}

Nature Structural and Molecular Biology, 2019, 26(7), 567-570. 


\section{ABSTRACT}

The cyclic enzymatic removal and ligation of the C-terminal tyrosine of $\alpha$-tubulin generates heterogeneous microtubules and affects their functions. Here we describe the crystal and solution structure of the tubulin carboxypeptidase complex between vasohibin (VASH1) and SVBP that folds in a long helix stabilising the VASH1 catalytic domain. The structure combined with molecular docking and mutagenesis experiments, reveals the residues responsible for recognition and cleavage of the tubulin C-terminal tyrosine. 
Microtubules (MTs) are key components of the eukaryotic cytoskeleton, involved in cell division, morphogenesis, motility and intracellular transport. Post-translational modifications of tubulin heterodimers, the so-called "tubulin code", includes the enzymatic removal and ligation of the C-terminal tyrosine ${ }^{(1)}$. While the tubulin tyrosine ligase (TTL) that reverts $\alpha$-tubulin to the translated form has been described ${ }^{(2)}$ and structurally characterised ${ }^{(3,4)}$, the carboxypeptidase removing it has remained elusive for four decades. Recently, we ${ }^{(5)}$ and others ${ }^{(6)}$ have described the first of the long sought tyrosine carboxypeptidases. The fully functional enzyme is a complex between SVBP, a 66-residue peptide, and vasohibins, VASH1 or VASH2. Expression of vasohibins increased detyrosination of $\alpha$-tubulin in cells, especially in the presence of SVBP. VASH1 has also been shown to remove the C-terminal tyrosine of $\alpha$-tubulin in vitro ${ }^{(5)}$. Vasohibins and SVBP have been implicated in neuronal differentiation, a function that may be associated with their role in tubulin detyrosination ${ }^{(6)}$.

As vasohibins, but not SVBP alone, affect the levels of detyrosinated $\alpha$-tubulin, and SVBP regulates the cellular abundance and solubility of vasohibins, SVBP was considered to have a chaperone-like function. VASH1 has been suggested having a transglutaminase-like protease fold, with a non-canonical Cys-His-Ser catalytic triad(7); however, low similarity to existing structures precludes a reliable structural model to be established. To establish the VASH1 fold, understand how SVBP exerts its function stabilising vashohibins, and how VASH1 specifically recognizes the $\alpha$-tubulin $C$-terminal peptide and cleaves the terminal tyrosine, we co-expressed a VASH1-SVBP complex in insects cells, purified it, and crystallised it. The crystal structure was determined by S-SAD phasing, notably by averaging 16 datasets of $360^{\circ}$ sweeps over different $\phi$ and $\chi$ angles using a PRIGo multi-axis goniometer ${ }^{(8)}$, to eradicate low-resolution noise by small satellite crystals. The structure was refined to $2.1 \AA$ resolution to an $R_{\text {free }}$ of $21.4 \%$ (see Online Methods and Table 1 for all crystallographic details).

While the purified VASH1-SVBP complex was expressed from a plasmid encoding VASH1 (1315 ) and the full-length SVBP peptide, only residues 60-304 of VASH1 and 26-52 of SVBP are visible in the refined electron density maps and modelled (Fig. 1a). The VASH1-SVBP complex has a compact structure (Fig. 1b, c): SVBP forms a slightly bent $\alpha$-helix that inserts between a short $\mathrm{N}$-terminal two-helix bundle domain of VASH1 (60-97) connected to an $\alpha / \beta$ fold C-terminal domain (118-304) comprised of seven $\alpha$-helices and a five-stranded antiparallel $\beta$ sheet flanked by helices, adopting a classic papain-like cysteine protease fold. An impressive $40 \%\left(1164 \AA^{2}\right)$ of the total surface area of SVBP is buried upon complex formation with VASH1. The interface between SVBP and VASH1 comprises 14 hydrogen bonds between the side chains of seven SVBP residues (Arg34, GIn35, Arg36, Glu38, Tyr40, Asn43, Thr47) and VASH1, and two salt bridges, both formed between Lys32 of SVBP and Glu163 of VASH1 (Fig. 1d). Notably, only two side chains of VASH1 (GIn133 and His136) interact with SVBP; all other VASH1 hydrogen bonds are through main chain atoms. The SVBP helix is very well conserved (Supplementary Figures 1,2 ), with the exception of a few residues that do not show any interactions with 
VASH1. The SVBP-interacting VASH1 surface is less well conserved, which may be rationalized by the observation that the VASH1 interaction interface predominantly involves main chain atoms. Mutations of key residues in SVBP, and most notably Lys32, Asn43, GIn35, Arg36, which make two or three hydrogen bonds to VASH1, reduced VASH1 expression and decreased detyrosination in HeLa cells co-transfected with both proteins (Fig. 1e; see Online Methods for details and Supplementary Fig. 3). This confirms a role of SVBP as a structural chaperone and highlights the importance of the specific interactions observed in the crystal structure interface between the folded VASH1 and SVBP domains.
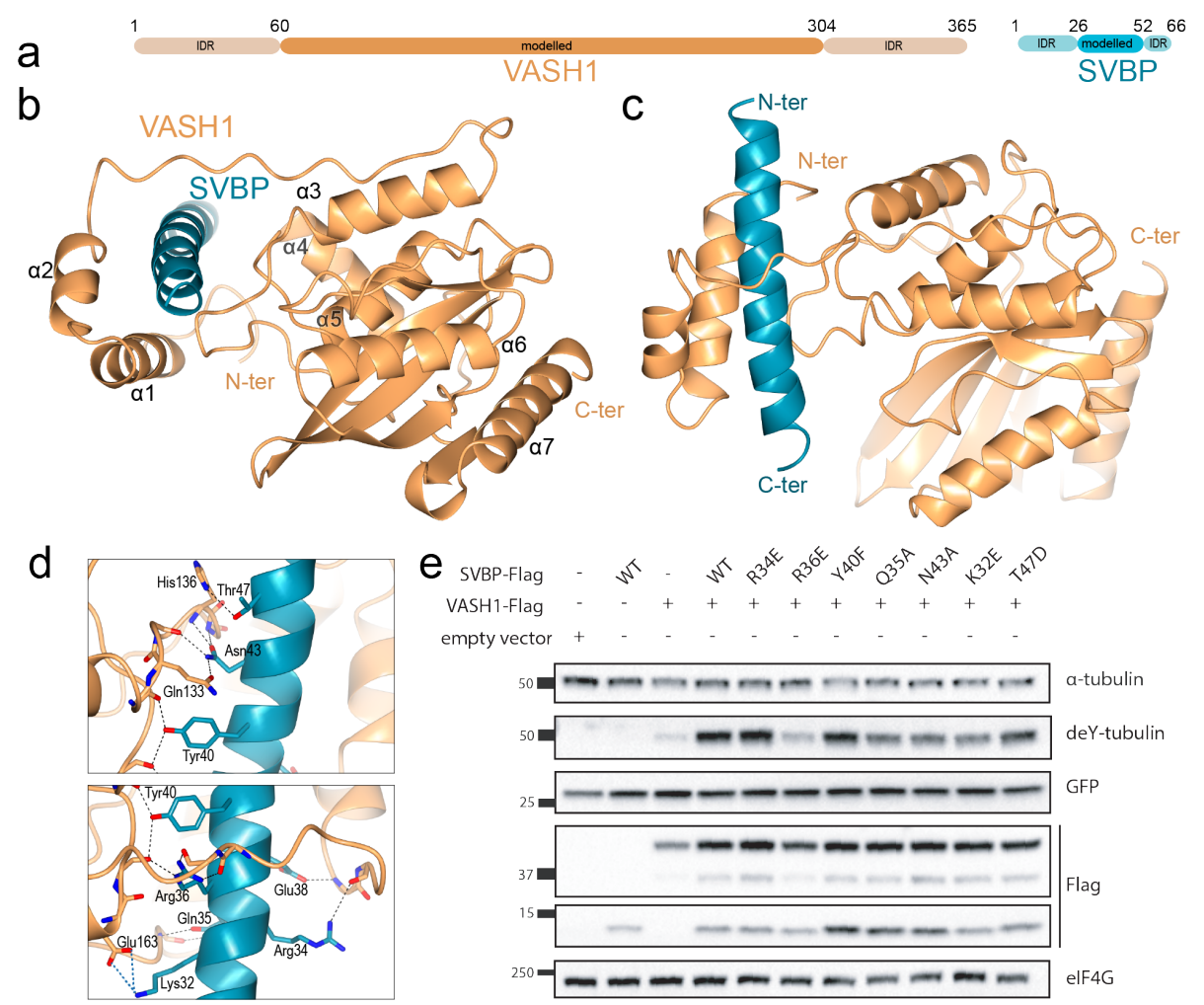

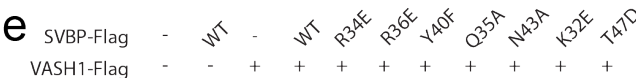

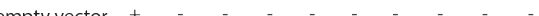

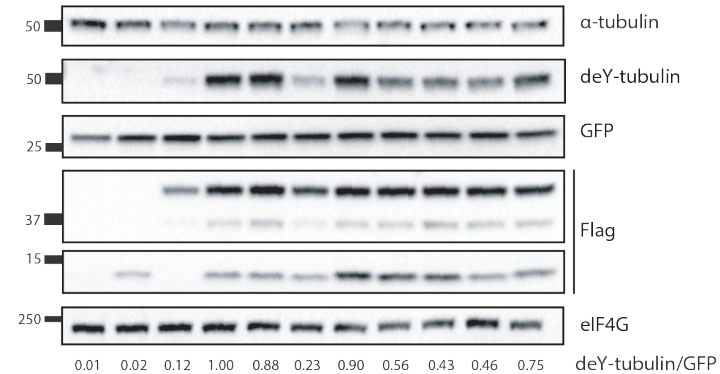

Figure 1. X-ray structure of the human VASH1-SVBP complex. (a) Domain organization of VASH1 and SVBP. (b, c) Ribbon representation of the structure. (d) Interacting residues in the complex interface shown as cylinders; the interaction network is shown as dotted lines (black: hydrogen bonds; blue: salt bridges). e, Western blots showing the expression and detyrosination effects of SVBP mutants in the VASH1 interface; quantification of detyrosination activity is based on two replicates (Supplementary Fig. 3); uncropped blot images are shown in Supplementary Data Set 1.

Comparing the VASH1-SVBP crystal structure with the small angle $X$-ray scattering (SAXS) data obtained from the full-length complex ${ }^{(5)}$, the fit is poor $(\chi=128$; Fig. 2a). Notably, the dimensionless Kratky plot (Fig. 2a inset) suggests a folded but elongated particle in solution ${ }^{(9)}$, 
as the maximum of the plot is not at the position expected for a well-folded and approximately spherical particle. VASH1-SVBP is a monomer in solution; a crystallographic dimer we observed is unstable and mutation of a key contact residue did not affect the function of VASH1-SVBP in cells (Supplementary Fig. 4). While the disordered N-terminus of VASH1 is poorly conserved and expectedly not well-ordered in our structure, it was more surprising that the well-conserved C-terminus (Supplementary Fig. 1) did not have a clear conformation. We used the BUNCH ${ }^{(10)}$ software to model missing regions to explain the elongated shape observed in SAXS data. The resulting full-length complex model in solution (Fig. 2a inset) fits excellent with the experimental data $(\chi=1.1)$, and suggests that all missing parts are unstructured and adopt extended conformations. This model does not fully explain the dimensionless Kratky plot, suggesting that both VASH1 and SVBP $\mathrm{N}$ - and C-terminal regions exist in multiple conformations ${ }^{(9)}$, behaving as intrinsically disordered regions (IDRs). We speculate that the conserved IDR C-terminus of VASH1 must be crucial for interaction with tubulin or other cellular factors.

The confirmed active site nucleophile of VASH1, Cys169 (mutated to alanine in our structure), resides within the VASH1 catalytic papain-like domain. It is positioned in the middle of a groove that is lined with several well-conserved residues (Fig. 2b), where positively charged Arg and Lys residues are predominant, resulting to a distinct positive electrostatic potential (Fig. 2c). As the $\mathrm{C}$-terminal tail of tubulin consists of several negatively charged residues (...EGEGEEEGEEY), this groove is ideally suited for substrate binding and catalysis. To understand in more detail the mode of substrate recognition, we first performed a scanning mutagenesis with a 12-mer tubulin tail peptide, were all glutamates were converted to alanine and the glycines were converted to proline to reduce structural flexibility. Analysis of the peptides after incubation with VASH1SVBP by semi-quantitative mass spectrometry (MS), allowed us to determine the ratio of intact versus de-tyrosinated peptides (Supplementary Fig. 5) based on the number of spectral counts of the intact peptides and their de-tyrosinated counterparts, respectively (see Online Methods for details). Surprisingly, the only mutation that affected substrate cleavage, other than the mutation to alanine or deletion of the terminal Tyr, was that of the second last glutamate (E-2). All other mutations were equally well processed as the wild-type terminal peptide.

With that information at hand, we used the high ambiguity driven biomolecular docking approach implemented in $\mathrm{HADDOCK}^{(11)}$, to suggest a binding mode for a short C-terminal peptide of tubulin (EGEEY) to VASH1. We used a fully flexible peptide, and restraints requiring the proximity of the terminal tyrosine and the crucial glutamate of the peptide to VASH1, and that the scissile bond should be recognised by the active site Cys169. We obtained several clusters of computational models representing the binding of the tubulin tail substrate to VASH1 (Supplementary Table 1). Using a representative model of the most populous cluster as a guide (Supplementary Fig. 6) we designed a series of site mutants of VASH1, to confirm the hypothesis for the catalytic triad residues(7) and the mode of binding of the substrate. We validated the activity of the VASH1 mutants by monitoring the level of detyrosination in HeLa 
cells transiently co-transfected with the VASH1 mutants and SVBP. In preliminary experiments, residues Lys146, Lys168, and Arg222 were shown to be important for activity. Based on that information, we performed a second round of modeling in HADDOCK, where along with the previous restraints we included as "active" residues for complex formation the latter three residues; we also used a longer fully flexible peptide (GEEEGEEY) as the model substrate. This new round of modeling strikingly suggested a unique cluster for the model of peptide binding (Fig. 3, Online Methods Table 2). This model was validated by additional mutagenesis experiments (Fig. 3 and Supplementary Fig. 7) that we discuss in detail.
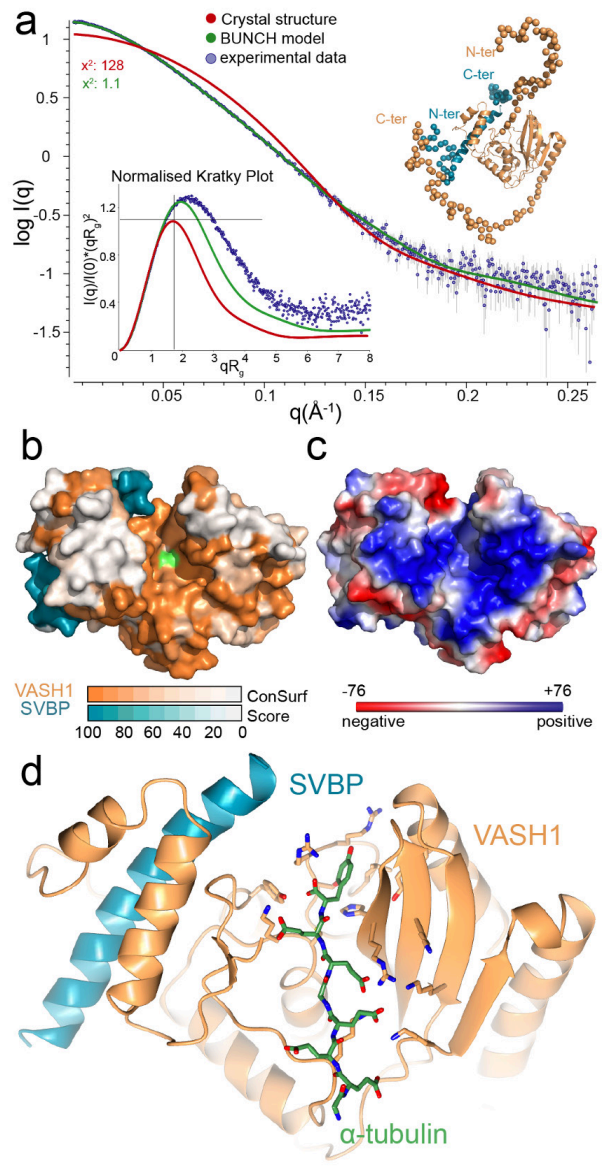

Figure 2. Structural analysis of the VASH1-SVBP complex. (a) Comparison of the SAXS data (blue), theoretical curve from the crystal structure (red), and the BUNCH model (green); the corresponding dimensionless Kratky plot is shown as an inset (the crosshair corresponds to the peak position for a well-ordered approximately spherical structure); the BUNCH model with the missing N- and C- termini shown as spheres also as an inset. (b) Surface representation of the VASH1-SVBP complex coloured by sequence conservation; the active site Cys169 is shown in light green (c) Surface representation of the VASH1-SVBP complex coloured by electrostatic potential $\left(\mathrm{K}_{b} \mathrm{~T} / \mathrm{e}_{\mathrm{c}}\right)$. (d) Ribbon representation of a repre-sentative computational model of the complex between VASH1-SVBP and the tubulin tail peptide (GEEEGEEY); the peptide and residues involved in binding and catalysis are shown as cylinders. 
The catalytic triad previously predicted ${ }^{(7)}$ includes the nucleophile Cys169 that is activated by His204; these predictions are supported by our model (Fig. 3b). Both the C169A and H204A mutations entirely abolished catalytic activity (Fig. 3c). The text-book mechanism ${ }^{(12)}$, and a structural search for folds similar to the VASH1 catalytic domain (Supplementary Fig. 8 and Online Methods), suggest that a negatively charged residue (Asp/Glu) stabilizes the protonated state of the histidine; such a negative charge is not obvious in VASH1, but might be provided by the main chain carbonyl of Leu226. The nucleophilic attack by Cys169, results to the release of the terminal tyrosine, leaving an anionic transient thioester intermediate, stabilised by a hydrogen donor; we believe that Ser221 could fulfil this role. Indeed, Ser221A has only $7 \%$ of the wild-type activity, consistent with a role in catalysis. We also note that in the structurally similar Pseudomonas avirulence AvrPphB protease ${ }^{(13)}$, this role is taken by Asn93 residue which is close to Ser221 (Supplementary Fig. 8e).

VASH1-SVBP acts as a carboxypeptidase specific for the C-terminal tyrosine. The terminal carboxyl group is predicted to make contact with Arg222 and Tyr134 in our model (Fig. 3d); consistently the R222E and Y134F mutants retain only $1 \%$ and $23 \%$ of the activity (Fig. 3e). According to our model, Lys168 could also be involved in recognition of the carboxyl group, or the E-1 glutamate of the tubulin tail. The K168E mutant retains only $10 \%$ of the activity; based on our MS results we hypothesize that the role of Lys168, is more important for the carboxyl recognition. The Tyr side chain does not show any obvious interactions in our model, with the exception of a weak hydrophobic packing interaction with Leu226. Mutation of Leu226 to alanine (L226A) does affect activity, reducing it to $45 \%$ compared to wild-type. This is consistent with our previous observation that a phenylalanine substitution in that position is well tolerated ${ }^{(5)}$. We note however, that the L226A substitution might also be affecting the positioning of the Leu226 main chain carbonyl and thus the stabilisation of the protonated state of His204. As also predicted by our model, the conserved R223A and Y247F mutants do not seem to significantly affect activity.

Our MS analysis indicated that the second last glutamate (E-2) is crucial for substrate recognition. Based on our model, E-2 is recognised by Lys146 (Fig. 3f); indeed, the K146E mutant retains only $19 \%$ of the wild-type activity (Fig. $3 \mathrm{~g}$ ). Other candidates for the E-2 recognition in our model could be the well-conserved Arg203 or Lys258; however mutation of these two residues to glutamate, affected activity by only $20 \%$. The double mutant of these two residues however, interestingly loses two thirds of the activity compared to wild-type, suggesting that the network of positive (VASH1) and negative (tubulin) residues is important, but has some degree of redundancy. Mutation of the Lys276 (contacting the E-4 glutamate) to glutamate, had no significant effect in activity, consistently with our MS analysis. 
a



C

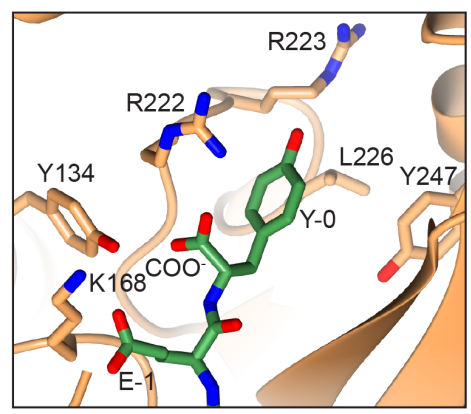

b



d

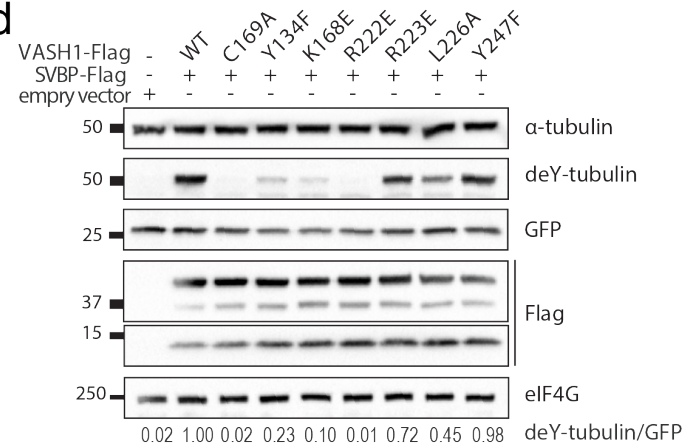

e

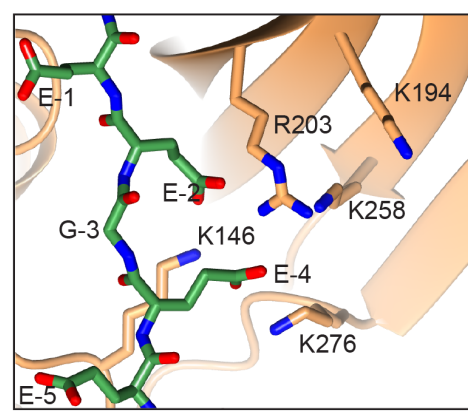

$f$

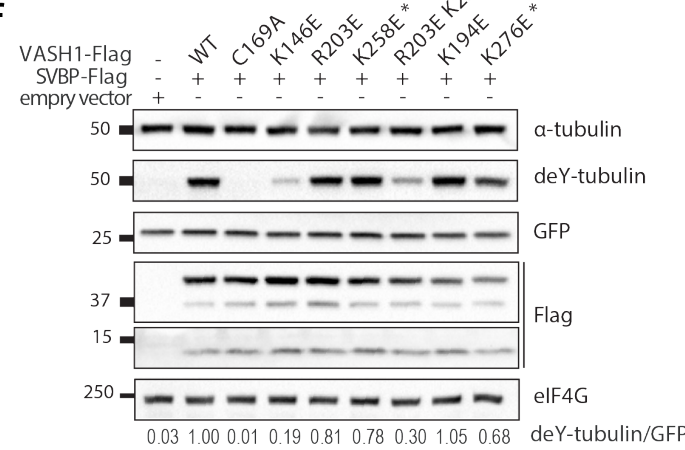

Figure 3. Tubulin tail binding and recognition. (a, c, e) close-up focusing on mutated residues whose activity in cells is shown respectively in (b) (active site residues), (d) (terminal tyrosine recognition) and (f) (glutamates recognition). Quantification of detyrosination activity is based on two replicates (Supplementary Fig. 7a-c); * denotes mutants that contained an additional mutation (H8OY) that has no effect on activity compared to the wild-type (Supplementary Figure 7d, e); uncropped blot images are shown in Supplementary Data Set 1.

Our crystal structure established that SVBP stabilises a helical N-terminal domain of VASH1 onto the catalytic papain-like cysteine protease fold. SAXS data imply the existence of wellconserved IDRs at the termini of both proteins. The computational model for binding of the tubulin tail we put forward has been validated by mutagenesis experiments and activity assays in cells. This model supports the catalytic triad and importantly suggests additional residues 
involved in substrate binding and recognition. Our structure also provides a scaffold for inhibitor or chemical probes design to further examine the function of tyrosine carboxypeptidases in the cell.

\section{Author contributions}

AA cloned, expressed, purified, and crystallised the complex, refined the crystal structure, analysed the SAXS data, wrote a draft manuscript and prepared figures. LL performed cell based experiments, analysed them, and prepared relevant figure panels. TH participated in al steps of protein purification, crystallisation and data collection. FT performed initial expression tests and produced the VASH1-SVBP complex that gave crystals, under the supervision of PC. $\mathrm{OBB}$ and MA analysed samples by mass spectrometry; these samples were prepared by JN. AP helped in structure determination and refinement, made final versions of the figures and the manuscript, and supervised the project together with TB.

\section{Acknowledgements}

We thank Vincent Olieric for help with S-SAD phasing and data collection at PXIII at the SLS Synchrotron, and the MASSIF beamline team at the ESRF for data collection. The mass spectrometry work was supported by The Netherlands Organization for Scientific Research (NWO) as part of the National Roadmap Large-scale Research Facilities of the Netherlands, Proteins@Work (project number 184.032.201) to M.A. 


\section{METHODS}

\section{Cloning expression and purification.}

A truncation construct of VASH1 (1-315) inactive mutant (C169A) and the full-length SVBP peptide, were expressed in Spodoptera frugiperda (Sf9) insect cells using the Invitrogen Bacto-Bac method in a way as described previously ${ }^{(5)}$. Briefly VASH1 was cloned in a modified pFastBac1 with a $3 \mathrm{C}$ cleavable C-terminal double-strep and $10 \mathrm{His}$ tag ${ }^{(14)}$. The complex was expressed in Sf9 insect cells using LONZA media. Cells grown in a shaker at $28^{\circ} \mathrm{C}$ and infected at a density of $2 \times 10^{6}$ cells $\mathrm{ml}^{-1}$. Cells were harvested $72 \mathrm{hr}$ post-infection, the biomass was flash frozen in liquid nitrogen, and stored at $-20^{\circ} \mathrm{C}$. The purification protocol described previously was used with the following modifications ${ }^{(5)}$. A two-step affinity purification scheme was employed, using first a Ni-NTA (Qiagen) affinity purification step, followed by Streptactin superflow resin (IBA) affinity purification. The tags were removed using 3C-protease overnight at $4^{\circ} \mathrm{C}$. The VASH1-SVBP complex was further purified by size-exclusion chromatography on a Superdex S-200 column (GE Healthcare) in $20 \mathrm{mM} \mathrm{HEPES/NaOH} \mathrm{pH} \mathrm{7.5,100mM} \mathrm{NaCl}$ and $1 \mathrm{mM}$ TCEP. The purified protein was concentrated with a $3 \mathrm{kDa}$ centrifugal concentrator (Amicon) to a final concentration of $3.5 \mathrm{mg} \mathrm{ml}^{-1}$, flash frozen, and stored at $-80^{\circ} \mathrm{C}$ until further use.

\section{Crystallization.}

Initial screening was performed using the commercial screens PACT, JCSG+, Classics-I \& II, ProComplex, ComPAS, pH-Clear, Morpheus. Hits were identified in the PACT screen $^{(15)}$ and further optimized using LIMBRO plates. VASH1-SVBP diffracting crystals were obtained in $0.1 \mathrm{M}$ Tris $\mathrm{pH} 8.8,0.2 \mathrm{M} \mathrm{CaCl}$, and 19\% PEG 3000. Crystals grew to maximum dimensions over a period of two weeks. Crystals were harvested and cryo-protected in a solution containing $30 \%$ glycerol and vitrified in liquid nitrogen. A native dataset was collected at beamline ID30A (MASSIF)(16) at the ESRF to $2.1 \AA$ resolution. Diffraction data were integrated and scaled automatically by XDS ${ }^{(17)}$ (Table 1 for statistics).

\section{Structure solution and refinement}

A native crystal was chosen for structure determination based on intrinsic sulphur anomalous signal. Using the PRIGo goniometer(8) we collected sixteen different datasets at a wavelength of 2.075 $\AA$ at the PSI X06DA beamline (PXIII) (Villingen, Switzerland). Each dataset was a full $360^{\circ}$ sweep with an oscillation angle of $0.2^{\circ}$ and a total exposure time of $180 \mathrm{sec}$ (with an estimated total dose of $0.5 \mathrm{MGy}$ ). The first ten datasets were collected stepping the chi angle by 5 degrees between datasets, and the six subsequent datasets were collected stepping the phi angle by 10 degrees between datasets. In each individual dataset the $\mathrm{R}_{\text {merge }}$ of the lowest resolution shell was between $7 \%$ and $12 \%$ and the signal over noise ratio of the anomalous signal was only slightly above unity. These discouraging statistics were most likely owing to the fact that smaller satellite crystals were present, giving rise to multiple lattices in the diffraction pattern at low resolution. Averaging all sixteen datasets, gave rise to a single dataset with 
an average multiplicity of seventy two times, giving rise to a low resolution shell signal over noise ratio of the anomalous signal close to three, extending to about $2.8 \AA$ resolution. The anomalous correlation coefficient was above 0.9 at the lowest resolution shell, and 0.3 at about $2.8 \AA$ resolution.

The HKL2MAP interface ${ }^{(18)}$ was used to run the SHELX(19) programs suite, using different resolution cutoffs and different number of sites. Looking for 8 sites at $2.8 \AA$ resolution, gave the most convincing solutions, with a few SHELXD trials having a higher correlation coefficient for both all and the weak reflections. The best solution identified nine sulphur sites with occupancy between 0.8 and 1.0, with the tenth site having an occupancy of 0.53 . We considered this a likely solution, and we run the CRANK pipeline ${ }^{(20)}$ using as input these sites. CRANK resulted in a model containing 280 residues in 6 fragments; $86 \%$ of the residues were docked in sequence. Using this model, we obtained a molecular replacement solution against the native dataset.

The structure was completed manually and refined by several iterations of REFMAC5 ${ }^{(21)}$ and manual rebuilding in COOT $^{(22)}$, including a few runs of the PDB-REDO pipeline ${ }^{(23)}$. The final model has an Rfree of 0.24 , is in the 100th percentile of Molprobity (0.82) and has a clashscore $(0.89)^{(24)}$ with no Ramachandran outlier.

Table 1. Crystallographic data

\begin{tabular}{|c|c|c|}
\hline & Native & S-SAD \\
\hline PDB identifier & $6 N V Q$ & $\mathrm{n} / \mathrm{a}$ \\
\hline \multicolumn{3}{|l|}{ Data Collection } \\
\hline Wavelength ( $\AA$ ) & 0.9660 & 2.075 \\
\hline Resolution $(\AA)$ & $47.6-2.10(2.21-2.10)$ & $50.0-2.8(2.91-2.8)$ \\
\hline Space Group & P 21212 & P 21212 \\
\hline Unit Cell a, b, c ( $(\AA)$ & $70.45,128.93,45.22$ & $70.58,127.76,45.01$ \\
\hline $\mathrm{CC}_{1 / 2}$ & $0.99(0.518)$ & $0.999(0.984)$ \\
\hline$R_{\text {merge }}$ & $0.062(1.538)$ & $0.157(1.057)$ \\
\hline$|1 / \sigma| \mid$ & $12.8(1.1)$ & $20.56(7.00)$ \\
\hline Completeness (\%) & $99.7(99.8)$ & $99.1(98.8)$ \\
\hline Multiplicity & $4.9(4.9)$ & $72(87)$ \\
\hline Unique Reflections & 24,696 & 19,243 \\
\hline \multicolumn{3}{|l|}{ Refinement } \\
\hline \multicolumn{3}{|l|}{ Atoms protein/other } \\
\hline B-factors Vash1/SVBP/Other $\left(\AA^{2}\right)$ & $59.9 / 65.2 / 60.1$ & \\
\hline$R_{\text {work }} / R_{\text {free }}(\%)$ & $18.9 / 22.6$ & \\
\hline Bond lengths rmsd $(\AA)$ & 0.0126 & \\
\hline Bond angles rmsd $\left({ }^{\circ}\right)$ & 1.772 & \\
\hline Ramachandran preferred/outliers (\%) & $97.4 / 0.0$ & \\
\hline Rotamers preferred/outliers (\%) & $88.3 / 4.58$ & \\
\hline Clash score (\%-ile) & $4.89(98)$ & \\
\hline MolProbity score (\%-ile) & $1.93(85)$ & \\
\hline
\end{tabular}

High Resolution shell in parentheses 


\section{Small Angle X-ray Scattering.}

Data for VASH1/SVBP complex were recorded and analyzed as described previously. BUNCH(10) from the ATSAS suite ${ }^{(25)}$ was used to add the missing residues to the crystal structure of VASH1/ SVBP using the default settings. Theoretical scattering data from VASH1 and SVBP atomic models were generated using $\mathrm{CRYSOL}^{(26)}$.

\section{VASH1 substrate specificity analysis by LC-MS/MS}

Peptides incubated with active VASH1 were analyzed on a Thermo Orbitrap Fusion hybrid mass spectrometer (Q-OT-qIT, Thermo Scientific) equipped with a Proxeon nLC 1000 system (Thermo Scientific, Bremen) fitted with a $75 \mu \mathrm{m} \times 500 \mathrm{~mm}$ analytical column (ReproSil-Pur 120 C18-AQ $2.4 \mu \mathrm{m}$ (Dr. Maisch $\mathrm{GmbH}$ ) packed in-house. Solvent A was $0.1 \%$ formic acid and peptides were eluted from the column in a 45-min gradient containing a 30-minute linear increase from 10$40 \%$ solvent B ( $80 \%$ Acetontrile/0.1\% formic acid). Survey scans of peptide precursors from $\mathrm{m} / \mathrm{z} 375-1500$ were performed at $120 \mathrm{~K}$ resolution in the Orbitrap with a $4 \times 10^{5}$ ion count target. Tandem MS was performed by HCD fragmentation with normalized collision energy of 20 and ion trap $\mathrm{MS}^{2}$ fragment detection. The $\mathrm{MS}^{2}$ ion count target was set to $10^{4}$ and the max injection time was set to $50 \mathrm{~ms}$. The instrument was run in top speed mode with 3 s cycles.

Raw data files were processed using Proteome Discoverer (version 1.4.0.288, Thermo Fisher Scientific). MS $^{2}$ spectra were searched against a custom database containing the mutagenized synthetic versions of the tubulin-like C-terminal peptides, using Mascot (version 2.6.1, Matrix Science, UK). No miscleavages were allowed and data was filtered with ion score $>20$. Determination of the extent of peptide cleavage by VASH1 -and subsequent assessment of VASH1 substrate specificity- was performed based on the number of peptide spectrum matches (PSM) for the intact and cleaved/detyrosinated version of each peptide.

\section{Docking}

A work-in-progress model of the VASH1-SVBP complex was used for a computational experiment using high ambiguity driven docking (HADDOCK ${ }^{(27,11)}$ ). First, we created an extended conformation of a minimal substrate peptide with the sequence EGEEY. For the docking we defined the terminal tyrosine and the second last glutamate as active residues for the substrate. For the VASH1-SVBP complex we only defined that the $\gamma$-S of the catalytic Cys 169 should be in contact with the carbonyl carbon of the scissile bond. All other parameters were used with default values. HADDOCK clustered 143 models in 9 clusters (Supplemental Table 1), of which the largest cluster contained 62 models. A representative of this cluster was used for designing validation experiments by site directed mutagenesis.

For the second modelling round first, we created an extended conformation of a minimal substrate peptide with the sequence GEEEGEEY. For the docking we defined the terminal tyrosine and the second last glutamate as active residues for the substrate. For the VASH1- 
SVBP complex we defined that the $\mathrm{Y}$-S of the catalytic Cys169 should be in contact with the carbonyl carbon of the scissile bond and Lys146, Lys168, and Arg222 as active residues. All other parameters were used with default values. HADDOCK clustered all 198 models in 1 cluster. The HADDOCK score for this cluster was-165.2, significantly better than-108.3 in the previous modelling round (Table 2 ).

Table 2. HADDOCK parameters for the only cluster in the second round of modeling

\begin{tabular}{ll}
\hline HADDOCK score & $-165.2+/-6.1$ \\
\hline Cluster size & 198 \\
RMSD from the overall lowest-energy structure & $0.5+/-0.3$ \\
Van der Waals energy & $-23.9+/-7.7$ \\
Electrostatic energy & $-701.8+/-46.0$ \\
Desolvation energy & $-4.7+/-10.5$ \\
Restraints violation energy & $37.2+/-2.20$ \\
Buried Surface Area & $1194.2+/-120.7$ \\
Z-Score & -1.7 \\
\hline
\end{tabular}

\section{Structure similarity searches}

Structure similarity searches were carried out by DALI ${ }^{(28)}$ searches, using a truncated version of the VASH1 catalytic core (residues 118-304) against the whole PDB. The top hits (Z-score >6.0) were analysed by manual inspection.

\section{Conservation analysis}

A multiple sequence alignment with sequences for VASH1 from different model organisms was prepared using CLUSTAL-OMEGA ${ }^{(29)}$ through the EBI service portal(30). We then used the ConSurf webserver ${ }^{(31)}$ to calculate the conservation scores, using default values and the Bayesian method. Visualization of the results was done with PyMOL.

\section{Mutagenesis}

VASH1 and SVBP site-specific mutants were created using the QuikChange strategy (Stratagene). All constructs were verified by sequencing.

\section{De-tyrosination assay}

The peptide detyrosination assay to determine substrate specificity of the VASH1:SVBP complex was performed as previously described in Nieuwenhuis et al. ${ }^{(5)}$, 500nM VASH1:SVBP complex was incubated with $500 \mu \mathrm{M}$ of the indicated peptide in buffer containing $50 \mathrm{mM}$ TrisHCL pH 8.0; 10\% glycerol; $1 \mathrm{mM}$ DTT; $1 \mathrm{mM}$ PMSF and was incubated for 2 hours at $37 \mathrm{C}$. All peptides were dissolved in General Tubulin Buffer (GTB) containing 80 mM PIPES pH 6.9, 2 mM $\mathrm{MgCl} 2$ and $0.5 \mathrm{mM}$ EGTA. 
To measure de-tyrosination activity in cells, constructs of Flag-tagged VASH1 and SVBP were transfected into $\mathrm{H} 1 \mathrm{HeLa}$ cells using lipofectamine 2000. To control for transfection efficiency between samples, a GFP-expressing plasmid was co-transfected. After washing with PBS, cells were harvested directly in sample buffer $(0.25 \mathrm{M}$ Tris- $\mathrm{HCl}$ pH 6.8; $6 \%$ SDS, 30\% Glycerol, 16\% $\beta$-mercaptoethanol and bromophenolblue). Boiled samples were run on $4-12 \%$ Bis-Tris gels in MOPS buffer. Blotting was performed using standard blotting buffer with $20 \%$ ethanol. After blocking in $4 \%$ milk in $0.1 \%$ TBS-Tween (blocking solution), blots were incubated with antibodies in blocking solution. Antibodies used were elF4G (Cell Signaling Technologies \#2498); $\alpha$ tubulin (DM1A; Santa Cruz Biotechnology \#32293), Flag (Cell Signaling Technologies \#2368), de-tyrosinated tubulin (Merck Millipore AB3201) and GFP (Santa Cruz Biotechnology \#8334). After membrane washing, blots were incubated with HRP-coupled secondary antibodies, washed and developed using standard ECL reagents.

De-tyrosination levels were quantified in the software Fiji. To measure the levels of GFP and de-tyrosinated Tubulin, same-sized rectangles were used to measure the raw integrated density (sum of pixel values) of each sample. The background (defined as the measurement in the rectangle on an empty area of the blot) was subtracted from each measurement and the de-tyrosinated Tubulin/GFP ratio was calculated. Ratios were normalized to the control (wildtype SVBP + VASH1).

Table 3. DALI search results with Z-score $>6.0$

\begin{tabular}{lllll}
\hline PDB ID & Z-score & RMSD & Coverage & \% Identity \\
\hline 4DMO & 7.6 & 3.1 & 124 & 12 \\
4FGP & 7.4 & 3.5 & 116 & 12 \\
2PQT & 6.4 & 3.1 & 125 & 15 \\
IUKF & 6.1 & 2.6 & 92 & 5 \\
4RXV & 6.1 & 3.7 & 126 & 5 \\
\hline
\end{tabular}




\section{REFERENCES}

1. A. Wehenkel, C. Janke, Towards elucidating the tubulin code. Nat. Cell Biol. 16, 303-305 (2014).

2. H. Murofushi, Purification and characterization of tubulin-tyrosine ligase from porcine brain. J. Biochem. 87, 979-984 (1980).

3. A. E. Prota et al., Structural basis of tubulin tyrosination by tubulin tyrosine ligase. J. Cell Biol. 200, 259-270 (2013).

4. A. Szyk, A. M. Deaconescu, G. Piszczek, A. Roll-Mecak, Tubulin tyrosine ligase structure reveals adaptation of an ancient fold to bind and modify tubulin. Nat. Struct. Mol. Biol. 18, 1250-1258 (2011).

5. J. Nieuwenhuis et al., Vasohibins encode tubulin detyrosinating activity. Science. 358, 1453-1456 (2017).

6. C. Aillaud et al., Vasohibins/SVBP are tubulin carboxypeptidases (TCPS) that regulate neuron differentiation. Science. 358, 1448-1453 (2017).

7. L. Sanchez-Pulido, C. P. Ponting, Vasohibins: new transglutaminase-like cysteine proteases possessing a noncanonical Cys-His-Ser catalytic triad. Bioinformatics. 32, 1441-1445 (2016).

8. S. Waltersperger et al., PRIGo: a new multi-axis goniometer for macromolecular crystallography. J Synchrotron Radiat. 22, 895-900 (2015).

9. R. P. Rambo, J. A. Tainer, Characterizing flexible and intrinsically unstructured biological macromolecules by SAS using the Porod-Debye law. Biopolymers. 95, 559-571 (2011).

10. M. V. Petoukhov, D. I. Svergun, Global rigid body modeling of macromolecular complexes against small-angle scattering data. Biophysj. 89, 1237-1250 (2005).

11. G. C. P. van Zundert et al., The HADDOCK2.2 Web Server: User-Friendly Integrative Modeling of Biomolecular Complexes. J. Mol. Biol. 428, 720-725 (2016).

12. H.-H. Otto, T. Schirmeister, Cysteine Proteases and Their Inhibitors. Chem. Rev. 97, 133-172 (1997).

13. M. Zhu, F. Shao, R. W. Innes, J. E. Dixon, Z. Xu, The crystal structure of Pseudomonas avirulence protein AvrPphB: a papain-like fold with a distinct substrate-binding site. Proc. Natl. Acad. Sci. U.S.A. 101, 302-307 (2004).

14. M. P. A. Luna-Vargas et al., Enabling high-throughput ligation-independent cloning and protein expression for the family of ubiquitin specific proteases. Journal of Structural Biology. 175, 113-119 (2011).

15. J. Newman et al., Towards rationalization of crystallization screening for small- to medium-sized academic laboratories: the PACT/JCSG+ strategy. Acta Crystallogr. D Biol. Crystallogr. 61, 1426-1431 (2005).

16. D. Nurizzo et al., RoboDiff: combining a sample changer and goniometer for highly automated macromolecular crystallography experiments. Acta Crystallogr D Struct Biol. 72, 966-975 (2016).

17. W. Kabsch, XDS. Acta Crystallogr. D Biol. Crystallogr. 66, 125-132 (2010).

18. T. Pape, T. R. Schneider, HKL2MAP: a graphical user interface for macromolecular phasing with SHELXprograms. J Appl Crystallogr. 37, 843-844 (2004).

19. I. Usón, G. M. Sheldrick, Advances in direct methods for protein crystallography. Curr. Opin. Struct. Biol. 9, 643648 (1999).

20. S. R. Ness, R. A. G. de Graaff, J. P. Abrahams, N. S. Pannu, CRANK: new methods for automated macromolecular crystal structure solution. Structure. 12, 1753-1761 (2004).

21. G. N. Murshudov et al., REFMAC5 for the refinement of macromolecular crystal structures. Acta Crystallogr. D Biol. Crystallogr. 67, 355-367 (2011).

22. P. Emsley, B. Lohkamp, W. G. Scott, K. Cowtan, Features and development of Coot. Acta Crystallogr. D Biol. Crystallogr. 66, 486-501 (2010).

23. R. P. Joosten, F. Long, G. N. Murshudov, A. Perrakis, The PDB_REDO server for macromolecular structure model optimization. IUCrJ. 1, 213-220 (2014).

24. V. B. Chen et al., MolProbity: all-atom structure validation for macromolecular crystallography. Acta Crystallogr. D Biol. Crystallogr. 66, 12-21 (2010).

25. M. V. Petoukhov et al., New developments in the ATSAS program package for small-angle scattering data analysis J Appl Crystallogr. 45, 342-350 (2012).

26. D. I. Svergun, B. C, K. M. H. J, CRYSOL-a Program to Evaluate X-ray Solution Scattering of Biological Macromolecules from Atomic Coordinates. 28 (1995), pp. 768-773.

27. C. Dominguez, R. Boelens, A. M. J. J. Bonvin, HADDOCK: a protein-protein docking approach based on biochemical or biophysical information. J. Am. Chem. Soc. 125, 1731-1737 (2003).

28. L. Holm, L. M. Laakso, Dali server update. Nucleic Acids Research. 44, W351-5 (2016).

29. F. Sievers et al., Fast, scalable generation of high-quality protein multiple sequence alignments using Clustal Omega. Mol. Syst. Biol. 7, 539 (2011). 
30. S. Chojnacki, A. Cowley, J. Lee, A. Foix, R. Lopez, Programmatic access to bioinformatics tools from EMBL-EB update: 2017. Nucleic Acids Research. 45, W550-W553 (2017).

31. H. Ashkenazy et al., ConSurf 2016: an improved methodology to estimate and visualize evolutionary conservation in macromolecules. Nucleic Acids Research. 44, W344-50 (2016). 


\section{SUPPLEMENTARY FILES}

Supplementary Table 1. Clusters and scores of $1^{\text {st }}$ round of Haddock modelling Cluster 1

\begin{tabular}{ll}
\hline HADDOCK score & $-108.3+/-6.3$ \\
\hline Cluster size & 62 \\
RMSD from the overall lowest-energy structure & $0.5+/-0.3$ \\
Van der Waals energy & $-30.6+/-4.2$ \\
Electrostatic energy & $-326.0+/-60.4$ \\
Desolvation energy & $-17.4+/-4.8$ \\
Restraints violation energy & $48.9+/-8.95$ \\
Buried Surface Area & $929.7+/-81.9$ \\
Z-Score & -1.7 \\
\hline
\end{tabular}

Cluster 2

\begin{tabular}{ll}
\hline HADDOCK score & $-100.5+/-6.9$ \\
\hline Cluster size & 21 \\
RMSD from the overall lowest-energy structure & $0.7+/-0.1$ \\
Van der Waals energy & $-31.1+/-5.2$ \\
Electrostatic energy & $-351.7+/-42.0$ \\
Desolvation energy & $-4.1+/-7.8$ \\
Restraints violation energy & $50.5+/-8.25$ \\
Buried Surface Area & $988.8+/-74.9$ \\
Z-Score & -1.2 \\
\hline
\end{tabular}

Cluster 6

\begin{tabular}{ll}
\hline HADDOCK score & $-99.7+/-7.6$ \\
\hline Cluster size & 7 \\
\hline
\end{tabular}

Cluster 4

\begin{tabular}{ll}
\hline HADDOCK score & $-81.3+/-5.7$ \\
\hline Cluster size & 9 \\
\hline
\end{tabular}

Cluster 8

\begin{tabular}{ll}
\hline HADDOCK score & $-80.6+/-10.3$ \\
\hline Cluster size & 6 \\
\hline
\end{tabular}

Cluster 5

\begin{tabular}{ll}
\hline HADDOCK score & $-78.1+/-6.3$ \\
\hline Cluster size & 8 \\
\hline
\end{tabular}

Cluster 9

\begin{tabular}{ll}
\hline HADDOCK score & $-73.7+/-15.3$ \\
\hline Cluster size & 4 \\
\hline
\end{tabular}

Cluster 3

\begin{tabular}{ll}
\hline HADDOCK score & $-73.5+/-4.4$ \\
\hline Cluster size & 20 \\
\hline
\end{tabular}

Cluster 7 


\section{VASH1}

1010

30

40

50

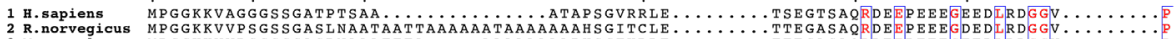

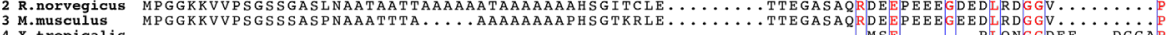

4 X.tropicalis
5 D.rerio
6 Vash2

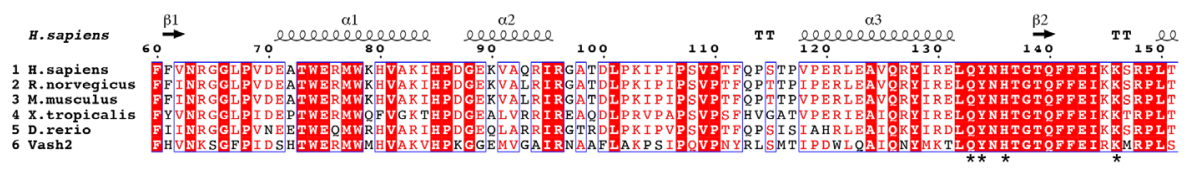

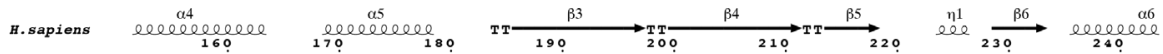

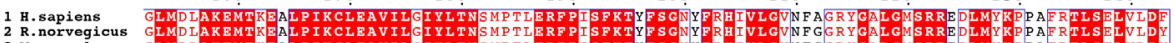

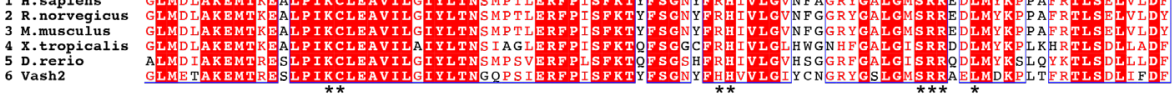

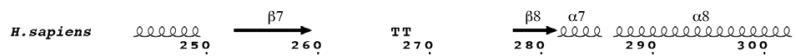

1 H. sapiens

2 R.norvegicus
3 M.musculus

3 M.musculus

5 D.rerio
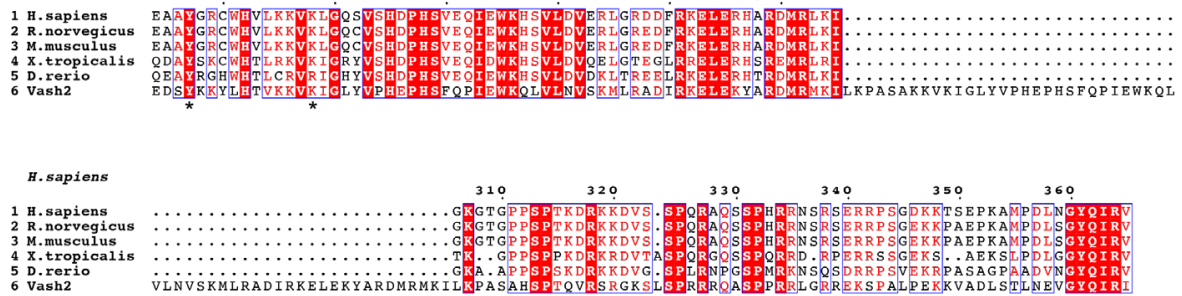

\section{SVBP}

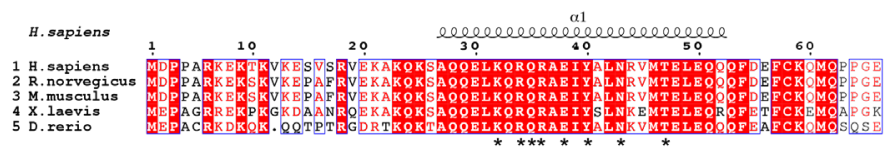

\section{Supplementary Figure 1. Conservation of VASH1 and SVBP sequence}

Multiple sequence alignment of orthologous proteins for VASH1 and SVBP. Structural elements are highlighted based on the obtained crystal structure for the VASH1-SVBP complex. 


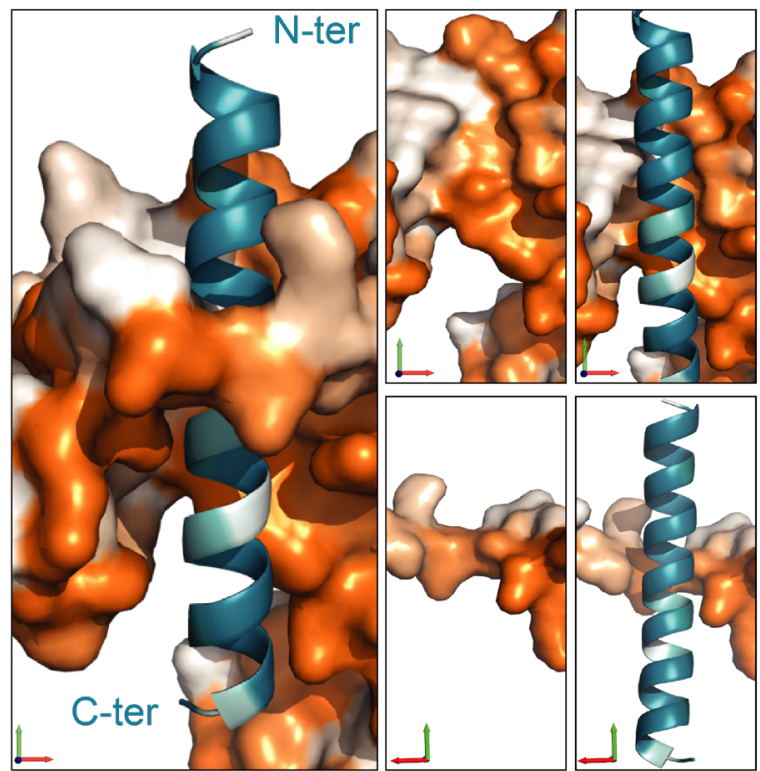

Supplementary Figure 2. Conservation of VASH1 and SVBP interface

Different views of the interacting surface between VASH1 and SVBP; VASH1 is shown as a surface, while SVBP is shown as ribbons; colours are according to conservation of the respective residues based on multiple sequence alignment of orthologous VASH1 proteins, as in Figure 2b. Axes are shown to compare the different views.

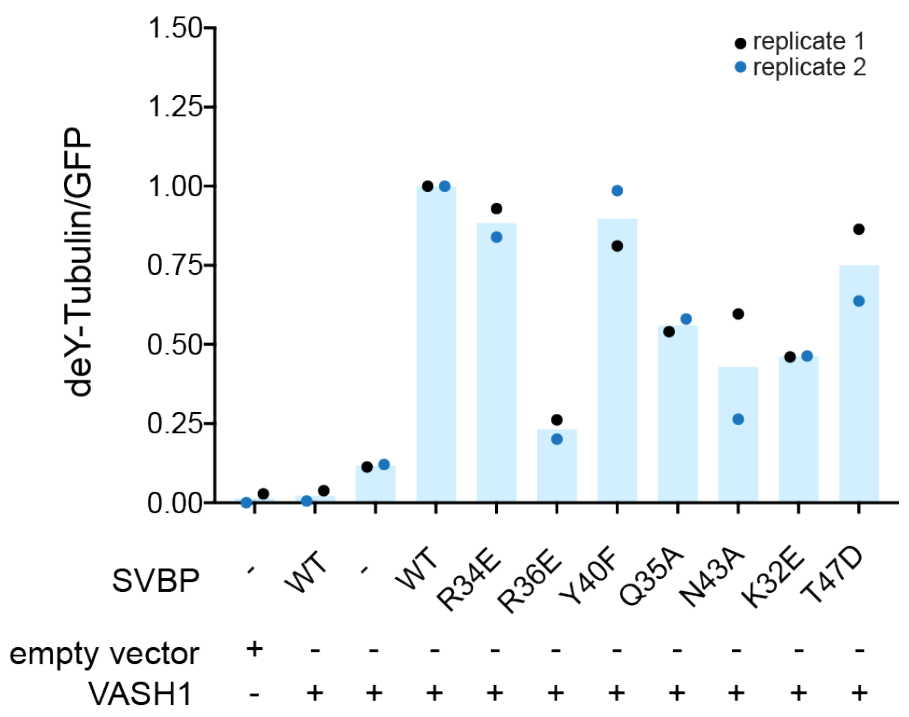

Supplementary Figure 3. Quantification of detyrosination activity of SVBP mutants

This graph relates to the quantification of activities shown in Figure 1. 




in HeLa cells

\section{Supplementary Figure 4. Detyrosination assay for crystallographic dimer contacts}

Detyrosination levels of HeLa cell lysates transfected with vectors expressing Flag-tagged SVBP, VASH1 and mutants were determined using western blotting.



Supplementary Figure 5. VASH1 substrate specificity analysis by LC-MS/MS.

Determination of VASH1 substrate specificity was performed based on the number of peptide spectrum matches (PSM) for the intact and cleaved/detyrosinated version of each peptide. 

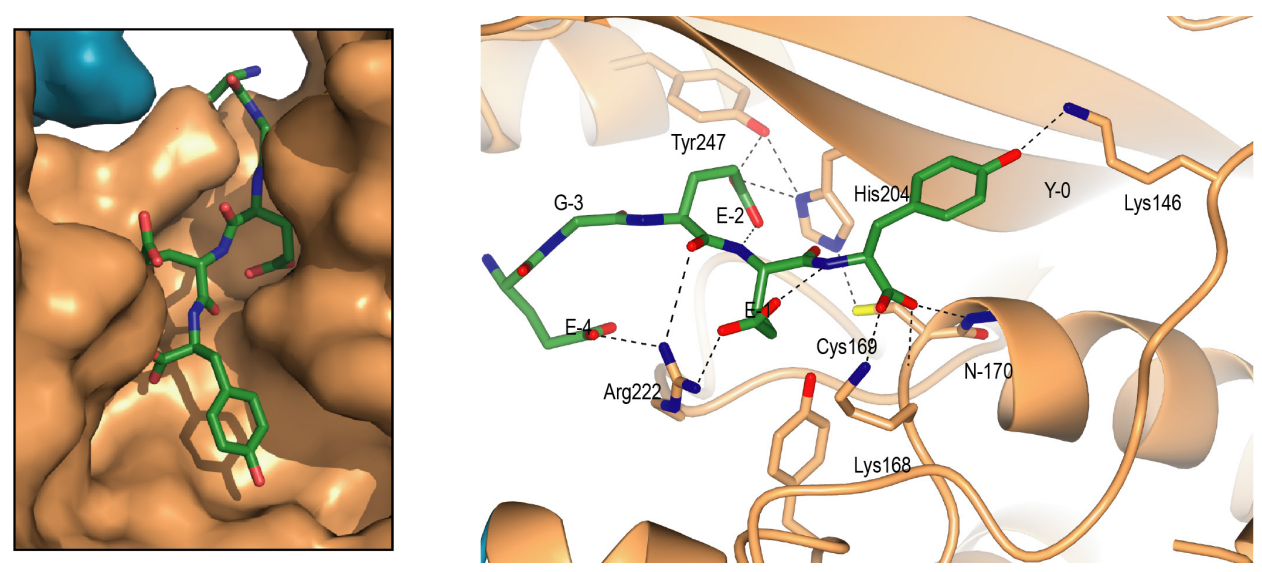

Supplementary Figure 6. Representative model of the most populous cluster from the first modeling round A representative model from Cluster 1 of the first round of HADDOCK modeling. This model proved wrong on the basis of the validation experiments but is shown here as it was used to guide the first round of validation experiments. 
a

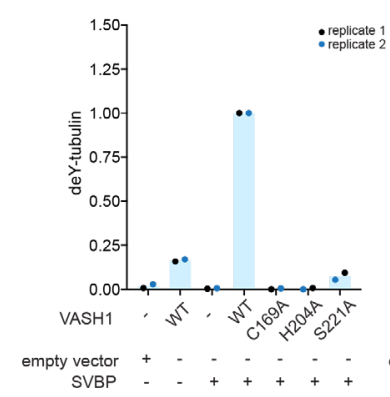

b

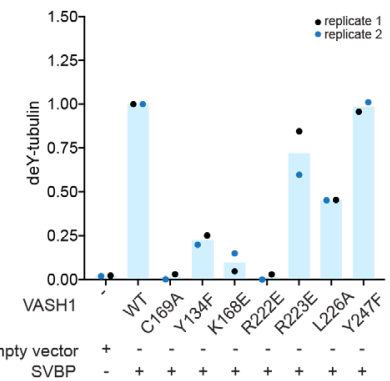

C

Referring to Figure $3 g$ d

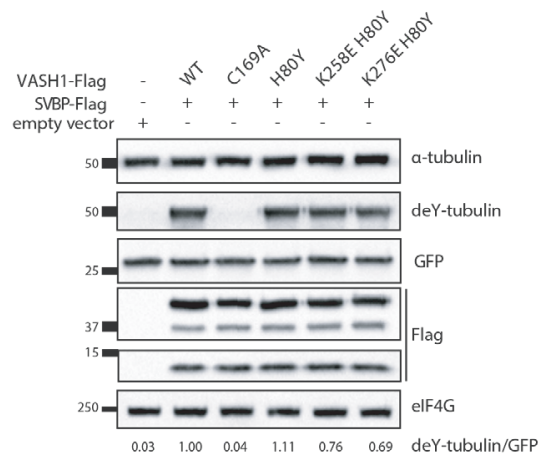

e

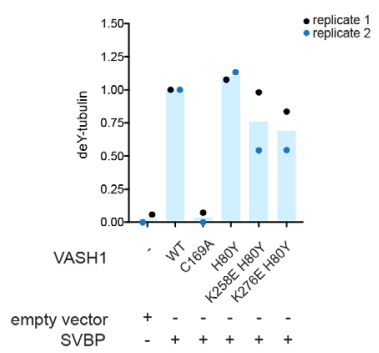

\section{Supplementary Figure 7. Quantification of detyrosination activity of VASH1 mutants}

The graphs in the first row relate to the quantification of activities shown in Figure 3; the bars present the mean and the two circles show the values of the two independent biological replicate experiments. The Western blot and the quantification in the second row, establishes that a mutation in His 80 that was noticed in two of our mutants shown with an asterisk in Figure $3 \mathrm{~g}$, does not affect activity compared to the wild type; in the same experiment we confirm again that the K258E and K276E mutants on this background, have no significant effect on activity. 
a
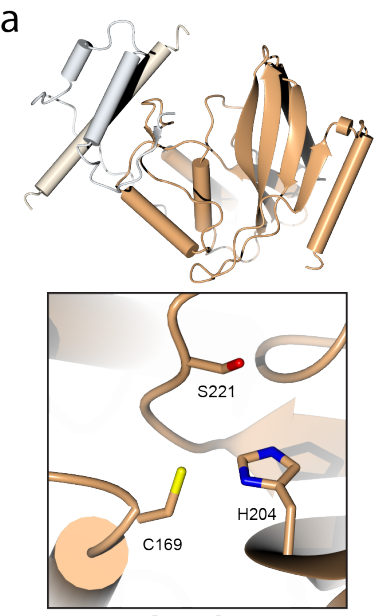

VASH1/SVBP

d
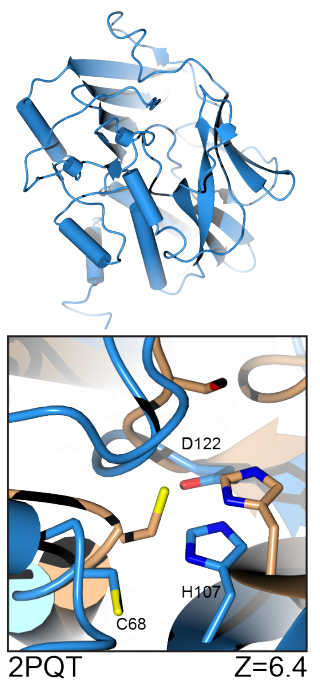

b
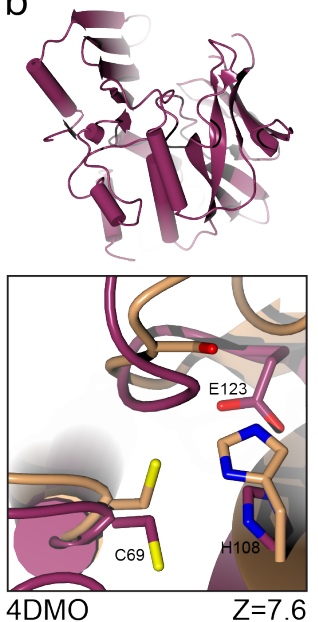

e
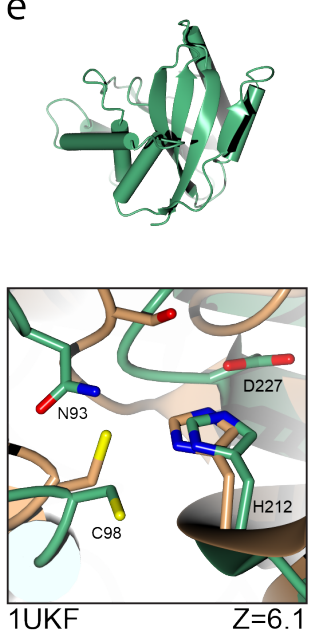

C
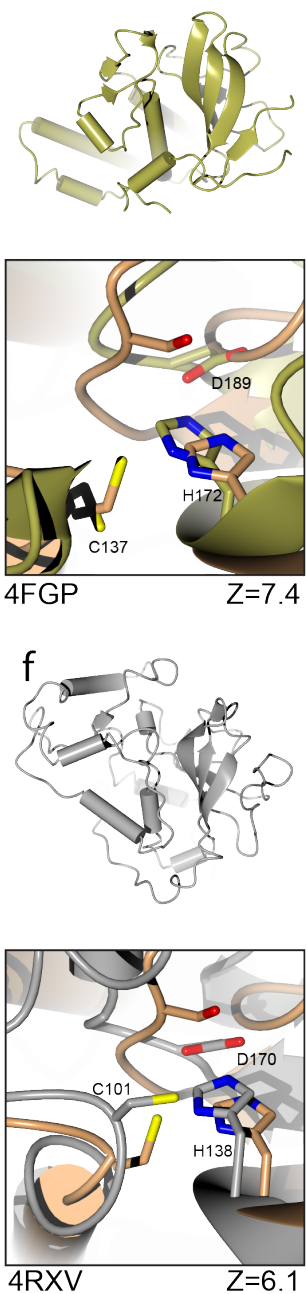

Supplementary Figure 8. Structural analysis of the active site of structural similar proteins to VASH1/SVBP complex Ribbon models of structural similar proteins based on DALI server; active site residues for each hit is shown as stick representation in all cases the catalytic triad contains a cysteine and a histidine, whereas the third position is occupied by either a glutamate or an aspartate; PDB codes are: $4 \mathrm{DMO}$ for Bacillus cereus arylamine $\mathrm{N}$-acetyltransferase 3 NAT3; 4FGP for LapG, 2PQT for human N-acetyltransferase-1 (the acetanilide molecule that has been covalently attached to the active site $\mathrm{C} 69$ is not shown in the figure); 1UKF for Avirulence protein from plant pathogen Pseudomonas syringae; $4 \mathrm{RXV}$ for RavJ protein fom Leugionella pneumophila. 



\section{Chapter 4}

Mechanism and structure of MATCAP a
new tyrosine metallo-carboxypeptidase 


\section{ABSTRACT}

Post-translational modifications of tubulin heterodimers lead to microtubule heterogeneity and therefore affect the function of microtubules. These modifications include the cyclic enzymatic removal and ligation of the $\mathrm{C}$-terminal tyrosine of $\alpha$-tubulin (detyrosination, tyrosination). The discovery of an $\alpha$-tubulin detyrosinating enzymatic complex, the vasohibins complex with SVBP, was a first step to understand the role of detyrosination in cells. However, detyrosination is still detected in vasohibin-deficient cells, indicating the existence of additional detyrosinating enzymes. Here, we build on the discovery of MATCAP as a novel enzyme in the detyrosination repertoire. Using a combination of biochemical, biophysical and structural methods, we present the characterization of MATCAP as a metal dependent carboxypeptidase, specific for the C-terminal tail of $\alpha$-tubulin, and describe the domain architecture and the crystal structure of the catalytic domain. Our findings will provide a structural framework for designing smallmolecule regulators to target detyrosination and may have therapeutic potential in human conditions with distorted tyrosination levels, such as cancer, heart diseases or brain disorders. 


\section{INTRODUCTION}

Microtubules are the largest filamentous components of the eukaryotic cytoskeleton ${ }^{(1)}$. They are composed of polymerized $\alpha-/ \beta$ tubulin heterodimers and are decorated with a number of modifications that account for their functional heterogeneity. These posttranslational modifications (PTMs), together with the plethora of isotypes for both $\alpha-/ \beta$ tubulin genes, are part of a broader regulatory repertoire known as the tubulin code ${ }^{(1)}$.

The detyrosination/tyrosination cycle has been the first PTM of tubulin, described four decades ago ${ }^{(2)}$. The enzyme regulating the initiating step of the detyrosination cycle, (the removal of tyrosine) has remained elusive until recently, when two independent research groups discovered the role of a complex comprised of the vasohibins (VASH1 and/or VASH2) and the small vasohibins binding protein (SVBP) in detyrosination, using a genetic approach ${ }^{(3)}$ or biochemical methods ${ }^{(4)}$. The discovery of this complex was followed by structural studies by seven independent research groups, leading to more than ten deposited crystal structures of VASH1/2 - SVBP complexes in the PDB ${ }^{(5-10)}$.

From the start however, it has remained clear that a residual detyrosination activity is still present in cells lacking both VASH1 and VASH2, which could not be attributed to the geneencoded detyrosinated form of $\alpha$-tubulin (TUBA4A). Based on these findings, we formulated the hypothesis that cells contain additional detyrosinating factors specific for $\alpha$-tubulin. To identify this unknown detyrosinating factor, Lisa Landskron in the group of Thijn Brummelkamp, searched for positive regulators of detyrosination. She used a similar genetic approach that resulted in the discovery of vasohibins, but this time the mutagenized cells that were vasohibindeficient, to find positive regulators independent of the vasohibins. The genetic screen identified MATCAP, a previously uncharacterized protein, as a potential detyrosinating enzyme (Fig-1). To further explore the potential role of MATCAP in the detyrosination cycle, MATCAP was overexpressed in HeLa cells (which show extremely low endogenous detyrosinated tubulin levels), and Western blot analysis confirmed that MATCAP detyrosinates tubulin in vivo (Fig-2a). To confirm the enzymatic detyrosinating activity of MATCAP, we used recombinant MATCAP protein, to perform in vitro detyrosinating assays, confirming the enzymatic role of MATCAP, as a tubulin modifying enzyme (Fig-2b). To check for additional detyrosinating factors, Lisa Landskron knocked out MATCAP in vasohibin-deficient HAP1 cells. This experiment suggested that depletion of MATCAP completely abrogates detyrosination, confirming MATCAP as the previously unidentified, and possibly the last, detyrosinating factor (Fig-2 c). 


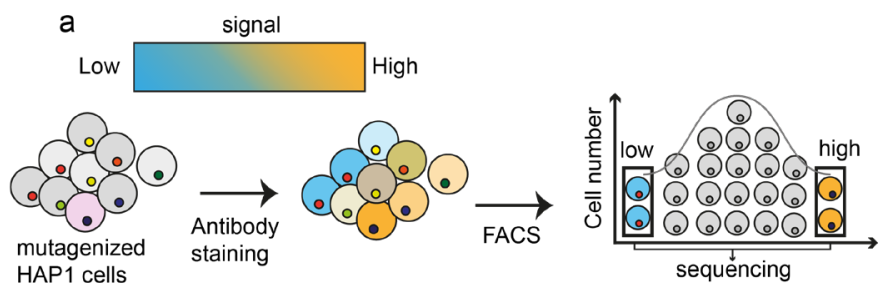

$b$ deY tubulin in $\triangle$ VASH1 $\triangle$ VASH2

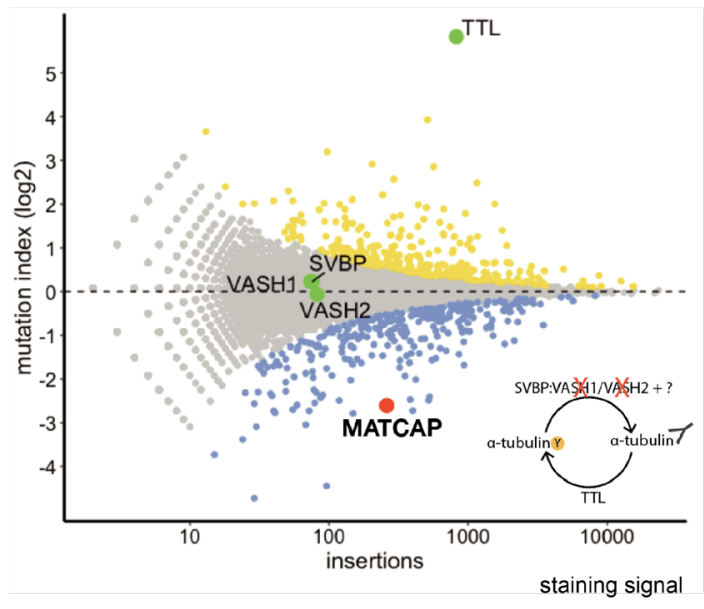

Figure 1. (a) The methodology used to identify positive and negative regulators using mutagenized HAP1 cells that have been stained with antibodies to detect the detyrosinated form of $\alpha$-tubulin; (b) "Fishtail plot" showing positive (in blue) and negative (in yellow) regulators of detyrosination in a vasohibin-deficient background. Mutations in genes that will result in a decrease in the deY $\alpha$-tubulin will reveal a positive regulator for detyrosination, whereas an increase in deY $\alpha$-tubulin will reveal the negative regulators. MATCAP appears as a strong positive regulator, whereas TTL is a negative regulator.

One intriguing characteristic of the detyrosination/tyrosination cycle is that the detyrosinating enzymes were not only unknown, but were not even characterized as proteases, based on their sequence. The crystal structure of the VASH1-SVBP complex set the basis for the characterisation of a Cys-protease as a detyrosinating enzyme. The finding of MATCAP, a metaldependent carboxypeptidase, suggests that MATCAP has presumably evolved for a similar function in a totally different structural scaffold and with a different enzymatic mechanism was intriguing. Thus, we set to first confirm that indeed MATCAP has a different structure and mechanism than the VASH tubulin carboxypeptidases we previously characterize. This could in turn allow us to answers additional questions of relevance to the tubulin code field. How does MATCAP recognizes and cleaves the $\mathrm{C}$-terminal tail of $\alpha$-tubulin? Why do cells contain different factors regulating the same procedures? 
a overexpression in HeLa cells

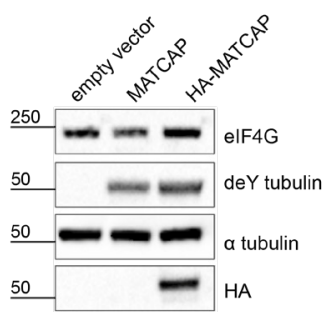

b in vitro recombinant protein



C

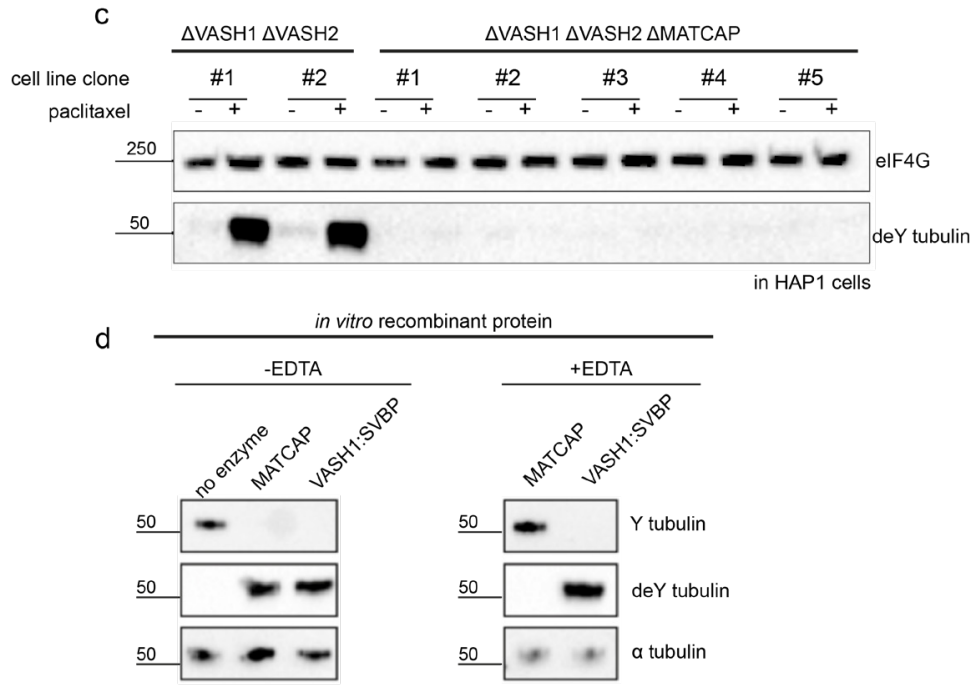

Figure 2. (a) Western blot analysis show that MATCAP overexpression in HeLa cells, detyrosinates $\alpha$-tubulin; (b) Recombinant MATCAP has in vitro detyrosination activity towards tubulin heterodimers further confirming its role as an additional detyrosinating enzyme.; (c) Depletion of vasohibins and MATCAP results in a complete loss of detyrosinating activity in HAP1 cells.; (d) In the presence of the chelating agent EDTA, MATCAP loses completely its detyrosinating activity, suggesting that a metal cation (possibly $\mathrm{Zn}^{2+}$ ) is necessary for its activity. VASH1:SVBP complex shows metalindependent detyrosinating activity, towards tubulin heterodimers.

Here, I present the structural analysis of the MATCAP protein, as a new tubulin detyrosinating enzyme. In vitro detyrosination experiments confirmed the metal-dependent nature of MATCAP. Small angle X-ray scattering (SAXS) experiments helped us to place the catalytic domain of recombinant MATCAP (MATCAP-CD) in the context of the full-length protein. We then generated crystals for MATCAP-CD, which diffracted to $2.1 \AA$ resolution. Our studies classify MATCAP as a bona fide detyrosinating enzyme and provide a framework for inhibitor or probe design for further studies of this new detyrosinating enzyme. 


\section{RESULTS}

\section{Expression and purification of MATCAP}

MATCAP full-length was cloned in a modified pFastbacl vector, containing a $3 C$ cleavable $\mathrm{N}$-terminal $10 \mathrm{His}$ double-strep tag ${ }^{(11)}$. The MATCAP recombinant protein was expressed in insect cells, purified through a Ni-NTA resin; the affinity tag was then removed by incubation with 3C-protease, followed by a size-exclusion chromatography (SEC) purification step The purified protein was concentrated to a final concentration of $15-20 \mathrm{mg} \mathrm{ml}^{-1}$, flash frozen and stored at $-80^{\circ} \mathrm{C}$ until further use.

\section{MATCAP is a metal dependent carboxypeptidase}

Experiments in cells have established the role of MATCAP as a potential carboxypeptidase towards microtubules. Protein sequence analysis suggested that MATCAP could likely require a metal ion for its catalytic activity. To examine this hypothesis, we performed in vitro detyrosination assays using the tubulin heterodimer as substrate, and recombinant protein purified from Sfg insect cells. The metal chelating agent ethylene-diamine-tetra-acetic acid (EDTA), was used at a concentration of $10 \mathrm{mM}$ to ensure that all possible metal ions that have been co-purified, are chelated. As a control we used the metal-independent VASH1/ SVBP complex under the same reaction conditions. The in vitro detyrosination results showed that in the absence of EDTA both MATCAP and the VASH1/SVBP complex can detyrosinate the $\mathrm{C}$-terminal tail of $\alpha$-tubulin. However, upon addition of EDTA the detyrosinating activity of MATCAP is completely abolished. This observation showed that MATCAP requires a metal ion (likely $\mathrm{Zn}^{2+}$ ) for its catalytic activity, and confirmed that MATCAP is a metal-dependent carboxypeptidase.

\section{The N-terminal of MATCAP is highly disordered}

Sequence analysis with the CCD webserver ${ }^{(12)}$ suggested that the first one third of MATCAP protein is disordered, followed by a globular core that consists of 330 residues. Disordered regions are usually highly flexible in solution and have an inhibiting role in structure determination. To structurally characterize MATCAP we decided to perform SEC-coupled Smal Angle X-ray Scattering (SEC-SAXS) experiments in solution, to understand the dynamics of the $\mathrm{N}$-terminal region and the $\mathrm{C}$-terminal catalytic domain.

SEC-SAXS measurement of the full-length recombinant protein (Fig. 4) suggested that MATCAP is an elongated molecule with a radius of gyration $(\mathrm{Rg})$ of 30.5 , and a maximal distance $\left(D_{\max }\right)$ of $123 \AA$. The pair distribution function (Fig 4c) has a characteristic shape where the tail to the right indicates some degree of flexibility due to a likely disordered region, which is attached to a main globular core. The presence of a disordered region was further confirmed by analysis of the normalized Kratky plot, as the curve is not smooth at high q*Rg range, indicating the presence of flexible/disordered regions in the full length MATCAP. The Kratky plot analysis 
also confirm the elongated space, as occurrence of the maximum deviates from 1.7 on the $x$-axis and from the expected maximum value on $y$, compared to these for a perfect sphere. Calculation of the MW of the protein using all available techniques for SAXS data (Porod, SAXSMoW2, Bayessian interference, $Q_{p}$, Size\&Shape) showed that MATCAP full-length is a monomer in solution, with the expected MW.

The hypothesis that MATCAP consists of a flexible $\mathrm{N}$-terminal region attached to a C-terminal globular compact protein core was thus established based on based on the secondary analysis and the SAXS data. We concluded that to elucidate the crystal structure of MATCAP we needed to remove the flexible $\mathrm{N}$-terminal region. We therefore designed a series of $\mathrm{N}$-terminal truncation constructs. Small-scale expression trials, were performed in Sf9 insect cells, to access the quality and quantity of the protein produced. The most promising construct, which we consider suitable for structural studies, was the one lacking the $\mathrm{N}$-terminal disordered region.

\section{SAXS analysis of MATCAP-CD identified a stable construct for crystallisation studies}

Removal of the first one third from the N-terminal, results in soluble protein that could be overexpressed in Spodoptera frugiperda insects cells and purified in milligram quantities. SAXS analysis for the MATCAP-CD construct revealed a more compact and globular molecule in solution; with a $D_{\max }$ of $85 \AA$. Analysis of the MW based on the SAXS data, suggested a monomer in solution. The Normalized Kratky plot for the MATCAP-CD construct, shows reduced flexibility compared to the full-length MATCAP, and almost reaches a maximum at 1.7. All the above are indicating that the truncated construct adopts a more compact globular fold without reduced flexibility. These characteristics defined MATCAP-CD as a promising candidate for crystallization studies.

\section{MATCAP recognizes and binds the C-terminal tail of $\alpha$-tubulin}

To study the affinity of MATCAP-CD towards its substrate, we performed binding studies to an $\alpha$-tubulin peptide, using fluorescence polarization (FP). We used a 10-residue peptide mimicking the C-terminal tail of $\alpha$-tubulin (TAMRA-GEGEEEGEEY), which was labeled with the tetra-methyl-rhodamine (TAMRA) fluorophore in the $\mathrm{N}$-terminus.

Titration of MATCAP-CD to the peptide, gave a clear increase in the FP signal, but not a clear plateau for the curve. The estimated $K_{D}$ was in the $\mu \mathrm{M}$ range $(28 \mu \mathrm{M})$ suggesting that the binding of MATCAP to the C-terminal tail of $\alpha$-tubulin is weak. That was in contrast to cellbased experiments showing that MATCAP recognizes and interacts with MTs. The low affinity to the deca-peptide could be possibly explained by the fact that MATCAP might recognize and interact other regions of the tubulin heterodimer and/or MTs. Since for the assays we used the active protein the presence of $\mathrm{Zn}^{2+}$ we can not exclude the possibility that the observed binding is, the binding of the MATCP-CD to the cleaved product (detyrosinated peptide) and not to the actual substrate. Further experiments are required to be performed to characterize 
biophysically the MATCAP-CD binding to the C-terminal tail of $\alpha$-tubulin.

a

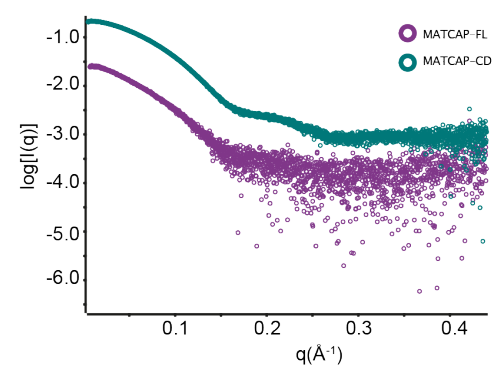

b

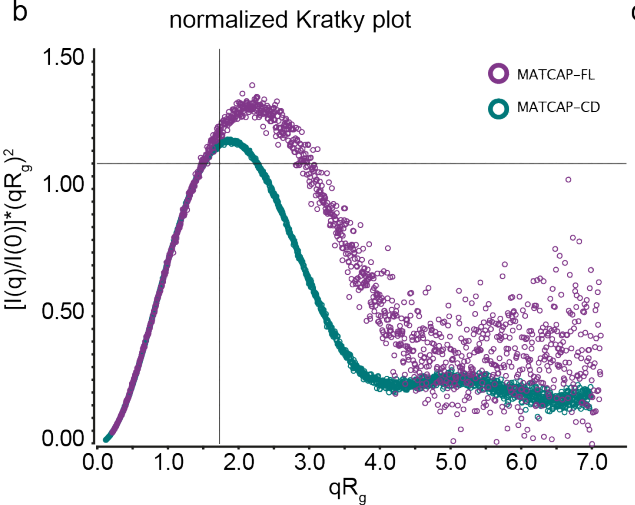

Guinier analysis

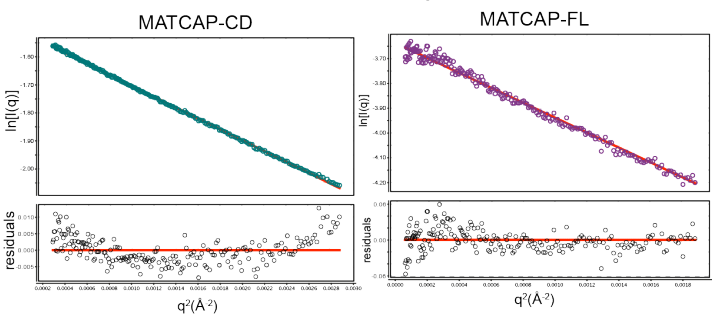

C

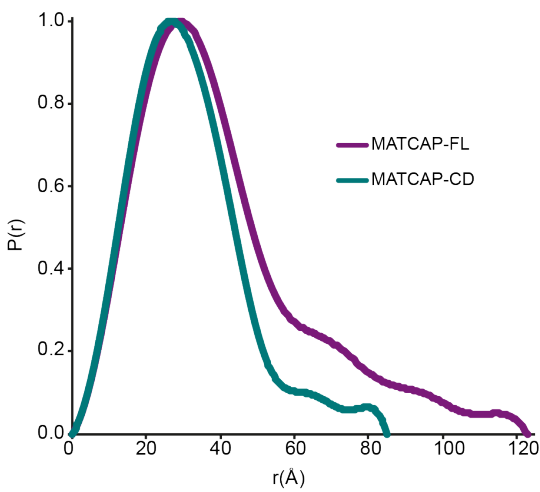

Figure 3. SAXS analysis of MATCAP full length (MATCAP-FL) and MATCAP-CD. (a) Experimental scattering intensities for both proteins and Guinier analysis for both MATCAP-FL and MATCAP-CD; (b) Normalized Kratky plot for full length MATCAP and MATCAP-CD. A perfect sphere would show a maximum at 1.7 on the $x$-axis, depicted by the crosshair; (c) Normalized pair distribution function

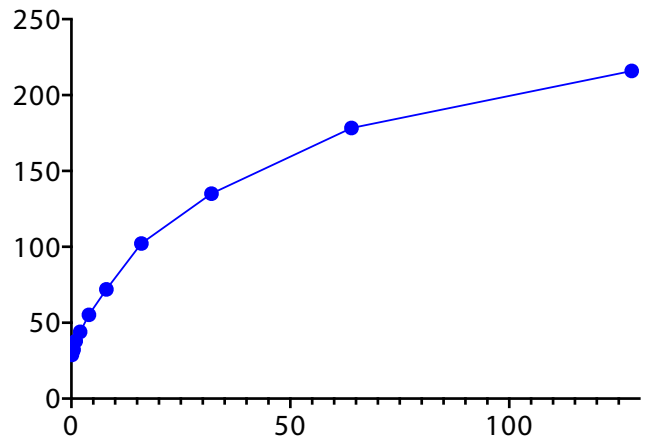

Figure 4. Fluorescence polarization binding assay of MATCAP-CD towards a deca-peptide mimicking the C-terminal tail of $\alpha$-tubulin 


\section{Towards diffracting quality crystals for MATCAP-CD}

MATCAP full length and MATCAP-CD were subjected to a number of crystallization trials using commercial screens. Although full length MATCAP did not give any hits, we were successful in obtaining initial hits for MATCAP-CD in the CLASSICS screen. The crystallization conditions contained $4.3 \mathrm{M}$ of $\mathrm{NaCl}$ and $0.1 \mathrm{M} \mathrm{HEPES} / \mathrm{NaOH}$ pH 7. The crystals appeared in multiple clusters. We extensively tried to optimize crystal morphology by micro- seeding, screening around the crystallization conditions, different incubation temperatures, protein to precipitant ratios, different protein concentrations. None of the above improved the size or morphology of the crystals. To improve crystal size and morphology, we used the additives screen from HAMPTON; in one condition, which contained EDTA as additive, we were able to reduce the number of clusters. After extensive rounds of optimization some of the clusters grew in size, containing well-defined 3D edges. We were successful in fishing single crystals by using the following approach: during harvesting we transferred a cluster that contained well defined shaped edges into the cryo-protectant solution; having the whole cluster into the cryo-protectant enabled us using the right loop, to break and harvest the growing end of the cluster. The above procedure could also be performed on the crystallization drop directly. However, it required extremely fast crystal-harvesting skills, since under the high concentration of precipitant (4.3M NaCl) salt crystals were formed almost immediately. Crystals diffracted to around $2.1 \AA$ resolution. While we were aware that the presence of EDTA as additive in the crystallization conditions would act as a chelating agent for the zinc ion, rendering the protein in a likely inactive conformation, for practical reasons we continued with these crystals for structure determination.

Due to lack of any similar structure in the PDB we were not able to solve the crystal structure using the molecular replacement approach. As expected, due to the presence of EDTA, the $\mathrm{Zn}^{2+}$ could not be used for obtaining initial phases (confirmed by $\mathrm{X}$-ray fluorescence scans around the $\mathrm{Zn}$ edge). S-SAD experiments were attempted but likely owing to the numerous sites (the asymmetric unit contained four monomers of MATCAP) we were unable to solve the structure this way. As diffraction quality crystals were grown only when MATCAP-CD was produced in insect cells, expression of Se-Met protein was not straightforward; and expression and purification of SeMet-substituted protein from bacteria, gave crystals diffracting at very low resolution. To successfully overcome the phase problem we thus soaked existing MATCAPCD crystals in different heavy atom solutions; a short dimethyl mercury chloride soak, was the one finally used to solve the phase problem. 

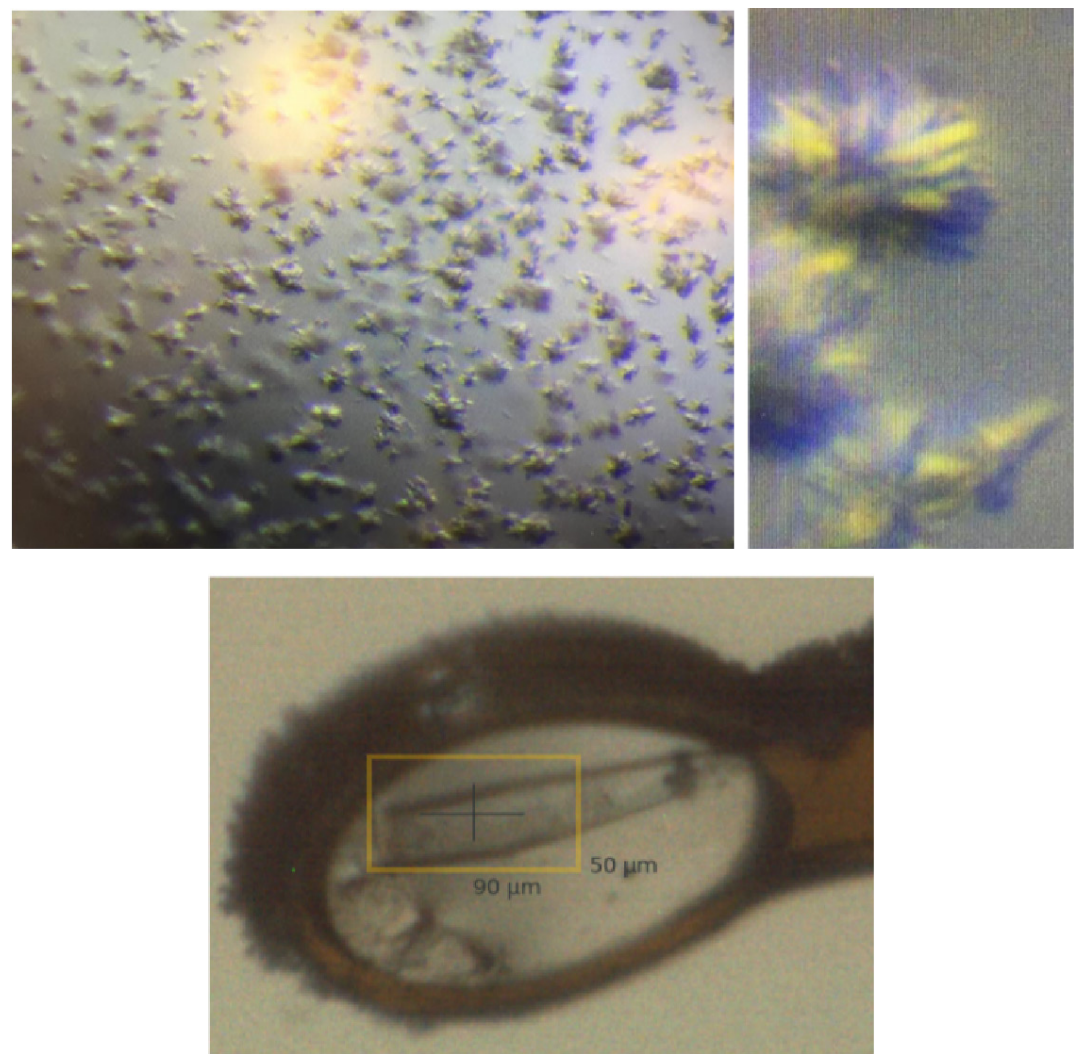

Figure 5. Crystals of MATCAP-CD. In the loop is the single crystal harvested using the procedure described in the main text. In the absence of EDTA crystals form clusters.

\section{Structure solution of MATCAP-CD}

Diffraction data of MATCAP-CD crystals were collected at PSI X06DA (PXIII, Villingen Switzerland) synchrotron using a PILATUS 2M-F detector. Crystals diffracted to $2.1 \AA$ resolution and belong to $P 2_{1}$ space group with unit cell parameters $a=55.85, b=88.03, c=165.62, b=90.67$. Crystal content analysis has indicated four molecules in the asymmetric unit, with a solvent content 52.6\%. The Hg derivative dataset was collected also in PSI X06DA (PXIII, Villingen Switzerland) and crystals belong to the same space group with very similar unit cell dimensions. Diffraction data were collected at $1.00 \AA$ wavelength at the $\mathrm{Hg}$ L-III edge, and gave a clear anomalous signal, extending to $3.8 \AA$ resolution, as judged by the statistics of the anomalous correlation during processing by XDS. To identify possible heavy atoms we used the HKL2MAP interface to run the SHELX suite with different resolution cut offs and expected number of heavy atom sites $^{(13)}$. The most convincing solution used data extending to a resolution of $4.2 \AA$, and gave ten sites $\left(\mathrm{CF}_{\text {best }} 71.2 \%, \mathrm{CC}_{\max } 43 \%\right)$. This solution showed four sites with occupancy between 1.0 and 0.88 , while the 10 th site had occupancy of 0.39 . The heavy atoms sites were used as input in the CRANK2 pipeline ${ }^{(14)}$, together with the native data set in a SIRAS experiment. This 
resulted in a model consisting of a total 1,300 residues in seven fragments; $90 \%$ of the residues were docked in sequence in the four monomers. The CRANK model was manually evaluated in COOT and one of the monomers in the asymmetric unit was manually completed. This monomer was used as an input model to obtain the placement of the other three monomers in the asymmetric unit. The structure was refined with several iterations of REFMAC ${ }^{(19)}$ and rebuilding in COOT ${ }^{(20)}$, including a few rounds of PDB-REDO ${ }^{(21)}$. The final model has an $\mathrm{R}_{\text {free }}$ of $25 \%$, and no Ramachandran outliers.

Later on, we have managed to obtain reasonably sized crystals of MATCAP-CD in the presence of $\mathrm{Zn}^{+2}$. These crystals diffracted X-rays $2.3 \AA$ resolution, and the structure was determined by molecular replacement from the apo structure. This structure clearly showed the bound zinc ion, and is refined to an $\mathrm{R}_{\text {free }}$ of $26 \%$, with no Ramachandran outliers.

\section{Crystal structure of MATCAP-CD}

The structure of MATCAP is comprised of 16 helices and three small antiparallel $\beta$-strands. The active site is located on the $\alpha$-helix that is perpendicular to the main core of helices. The structure of MATCAP can be divided into two major lobes, separated by a groove as depicted in the structure when the helices are shown as ribbons (Fig-7a-d). The surface representation of the structure reveals a cylindrical tunnel at exactly where the groove is around the main helix perpendicular to the three $\beta$-strands. This is also the area of the active site, where the $\mathrm{Zn}^{2+}$ catalytic ion is located. Positively charged residues surround the area around the metal ion. This is somehow similar to the VASH1/SVBP complex, where a positively charged network is responsible for binding and recognition of the C-terminal negatively charged tubulin tail. A surprising finding is that MATCAP-CD has near the active site a tunnel, compared to the positively charged groove that is present in VASH1/SVBP. However, the positively charged patch suggests that both detyrosinating enzymes could share the same basic principles for binding and recognition their substrate.

A comparison of the MATCAP structures in the presence and absence of the $\mathrm{Zn}^{2+}$ bound ion, shows that the beginning of the $\mathrm{N}$-terminal region is folded, adopting a helical turn conformation (Fig7a-b) located in front of the tunnel. This short helical turn could block the entrance to the tunnel; this can be visualized when both structures are colored according to their electrostatic potential. We can speculate that this might suggest an opening/closing mechanism where in the presence of $\mathrm{Zn}^{2+}$ ion, the structure is in its active conformation, but entrance to the active site is blocked by a short helix. Upon interaction with the tubulin C-terminal tail, MATCAP-CD could orient the tail of $\alpha$-tubulin in the active site to be detyrosinated. Although this is an interesting hypothesis, further experiments and perhaps new structures are required, where the $\mathrm{N}$-terminal region could be extended, to shed light on the opening/closing mechanism of MATCAP-CD. 
Experiments to characterize the MATCAP binding site in detail and determine the peptidebinding mode are ongoing, but have not been finished and are outside the scope of this chapter and my thesis, due to time constraints and practical considerations. I sincerely hope that I will be in position to discuss such data in my public defense.

a

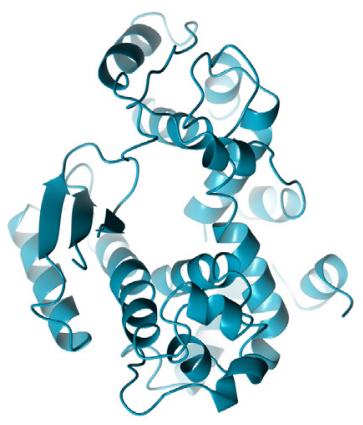

C

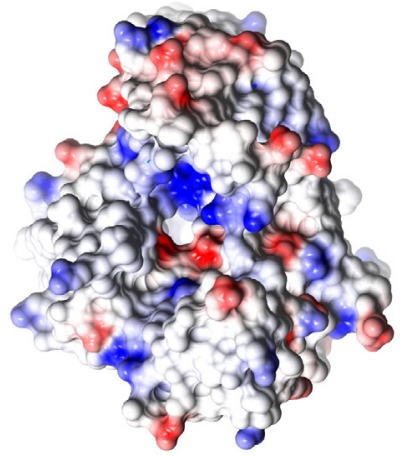

b

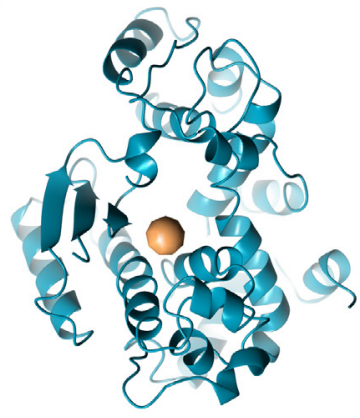

d

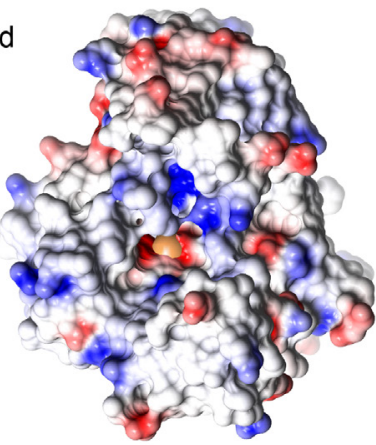

Figure 6. (a) Crystal structure of MATCAP-CD; (b) Structure of MATCAP-CD where the $\mathrm{Zn}^{2+}$ ion is depicted as a golden sphere in the active site; (c, d) Structure of MATCAP-CD colored according to electrostatic potential; the tunnel surrounded by positively charged residues.

\section{MATCAP-CD is more dynamic in solution based on SAXS measurements}

In general crystal structures depict in atomic detail a structural snapshot of a biomolecule at a certain conformation (inactive, intermediate, active state). Proteins are dynamic macromolecules that adopt multiple conformations in solution. Since we had collected SECSAXS data and solved the crystal structure of MATCAP-CD, we wanted to identify how dynamic MATCAP-CD is in solution, compared to the crystal structure. A direct comparison between the calculated scattering intensities of MATCAP-CD crystal structure with the experimental intensities from the data in solution, suggests that MATCAP-CD adopts a more compact fold in the crystal, and this behavior is independent of the presence or absence of $\mathrm{Zn}^{2+}$ from the protein structure. We suspect that this behavior is due to crystal packing. The characteristic 
shape of the $P(r)$ and the normalized Kratky plot also confirms the existence of flexible structural elements in MATCAP-CD in solution, which explains the difference in $x^{2}$ between the crystal structure and solution scattering data.

Therefore, we analyzed the crystal structure of MATCAP-CD for the presence of possible structural elements that are more dynamic in solution and lose their flexibility upon crystal packing. To evaluate the MATCAP-CD crystal structure for the presence of these structural flexible elements we used the program SREFLEX from the ATSAS suite. SREFLEX is a pipeline that follows a normal mode analysis (NMA) after partitioning the crystal structure into pseudodomains, creating a pool of models that represent different conformational changes that MATCAP might undergo. Scattering profiles are calculated for each model and compared with the experimental scattering profile, based on the $x^{2}$ method.
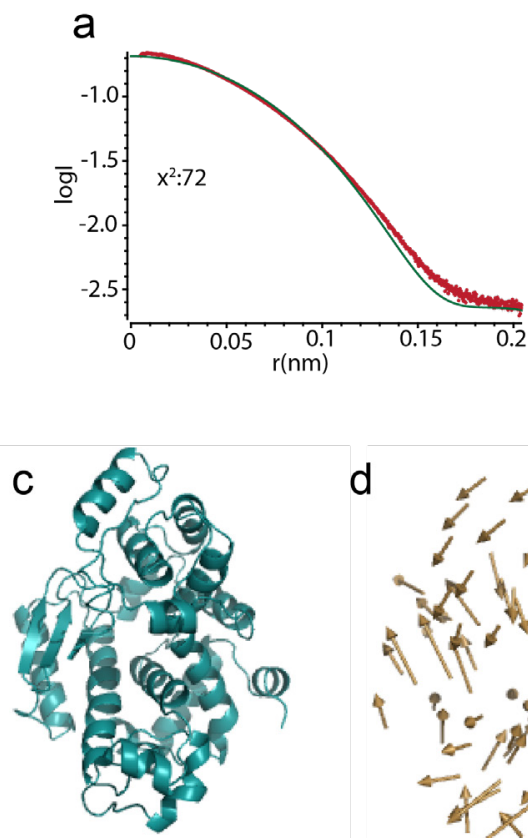

b

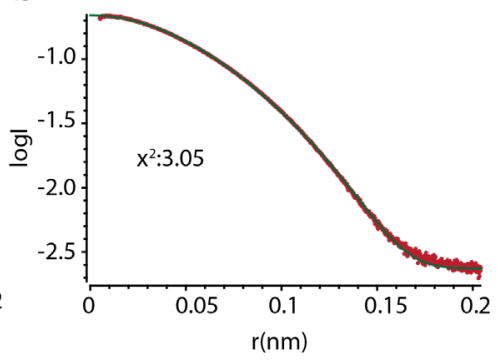



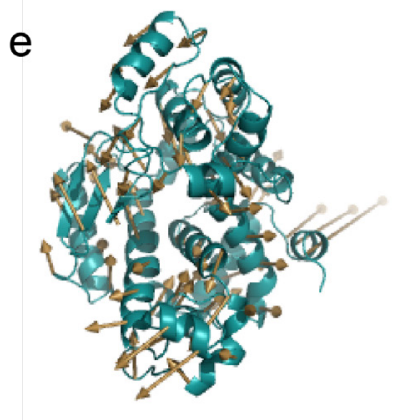

Figure 7. (a) Comparison of the SAXS data (red), with the theoretical curve from the crystal structure (green), before SREFLEX analysis. (b) After SREFLEX analysis; the $\mathrm{x}^{2}$ is indicating as inset; (c) Crystal structure of MATCAP-CD; (d) Vectors show the transition modeled by SREFLEX analysis of the MATCAP-CD crystal structure, guided by the SAXS data in solution.

The SREFLEX analysis suggests that the overall shape of MATCAP-CD might be more compact in the crystal structure than in solution, in which the protein is certainly more flexible. Based on the NMA analysis most of the helices are undergoing minor to major movements, which 
indicates that due to the crystal packing, some of the helices might have been packed close to the central globular core. The majority of the changes that are calculated by SREFLEX, are located in the C-terminal region of MATCAP and on the helical parts close to the small antiparallel b-sheet. This is an indication that the dynamic nature of MATCAP-CD is not fully depicted by its crystal structure. In solution, some helical parts of MATCAP-CD that might interact with other proteins, and/or tubulin could be in multiple conformations, explaining the difference in $x^{2}$ between the crystal structure and the experimental SAXS data. Notably, manual inspection of the crystal structure revealed that the last 30 residues are folded as a helixloop-helix motif, which according to the SREFLEX analysis, undergoes the largest movement in solution. This could explain the characteristic shape of the $P(r)$ distribution function and of the normalized Kratky plot. The role of the C-terminal region should be explored for interactions with other proteins or with the tubulin heterodimer. 


\section{MATERIALS AND METHODS}

\section{Protein production}

After an in-depth secondary structure analysis using proteinCCD ${ }^{(12)}$ and Quick2D ${ }^{(17)}$ we came into the conclusion that MATCAP contains a highly disordered N-terminal region. Therefore, a series of truncation constructs were designed and cloned to a modified pFastBac1 with a $3 \mathrm{C}$ cleavable N-terminal $10 \mathrm{His}$ double-strep tag ${ }^{(11)}$. Truncated recombinant protein was expressed in Spodoptera frugiperda insect cells using the Invitrogen Bac-toBac method as described previously ${ }^{(5)}$. MATCAP stable truncation was expressed in Sf9 insect cells using Insect-Xpress media (LONZA). Cells were grown in a shaker at $28^{\circ} \mathrm{C}$ and infected at a density of $2 \times 10^{6} \mathrm{cells} \mathrm{ml}^{-1}$. Cells were harvested $56 \mathrm{~h}$ post-infection, and the biomass was flash frozen in liquid nitrogen and stored at $-20^{\circ} \mathrm{C}$. A one-step affinity purification scheme was employed similar to the one described previously ${ }^{(4)}$, using first a Ni-NTA (Qiagen) affinity purification step, followed by removal of the affinity tag using $3 \mathrm{C}$-protease overnight at $4^{\circ} \mathrm{C}$. MATCAP was further purified by size-exclusion chromatography on a Superdex S-200 column (GE Healthcare) in $20 \mathrm{mM}$ $\mathrm{HEPES} / \mathrm{NaOH} \mathrm{pH} 7.5,140 \mathrm{mM} \mathrm{NaCl}$ and $1 \mathrm{mM}$ Tris(2-carboxyethyl)phosphine $1 \mu \mathrm{M} \mathrm{ZnCl}$. The purified protein was concentrated with a $10 \mathrm{kDa}$ centrifugal concentrator (Amicon) to a final concentration of $15-20 \mathrm{mg} \mathrm{ml}^{-1}$, flash frozen and stored at $-80^{\circ} \mathrm{C}$ until further use.

\section{Fluorescence polarization binding assays}

To measure the affinity of MATCAP to the $\alpha$-tubulin tail, an N-terminal tetramethylrhodamine (TAMRA) 10meric peptide (TAMRA-GEGEEEGEEY) was incubated with a titration range of MATCAP. Assays were performed in buffer containing (20mM HEPES-NaOH pH 7.4, 140mM $\mathrm{NaCl}, 1 \mathrm{mM}$ TCEP, 0.05\% Tween-20, 5mM EDTA) on a Pherastar plate reader (BMG, LABTECH, $\mathrm{GmbH}$, Germany) using excitation wavelength $540 \mathrm{~nm}( \pm 20 \mathrm{~nm})$ and detection of polarization at $590 \mathrm{~nm}( \pm 20 \mathrm{~nm})$. The anisotropy of TAMRApeptide was calibrated at $35 \mathrm{~mA}$ any change in anisotropy upon interaction with MATCAP was calculated using MARS software analysis and plotted in GraphPad Prism 8.

\section{In vitro detyrosination assay}

In vitro assays were performed incubating 500nM enzyme with 50nM of HeLa tubulin (Cytoskeleton) in a reaction volume of $30 \mu \mathrm{l}$, in buffer containing $50 \mathrm{mM}$ Tris- $\mathrm{HCl} \mathrm{pH} 8.0$, $10 \%$ glycerol, $1 \mathrm{mMDTT}, 1 \mathrm{mM}$ PMSF and was incubated for 2 hours at $37^{\circ} \mathrm{C}$. Samples were mixed with sample buffer and subjected to immunoblotting using antibodies recognizing the detyrosinated form of $\alpha$-tubulin. Antibodies used for immunoblot analysis: elF4G (Cell Signaling Technologies \#2498); $\alpha$-tubulin (DM1A; Santa Cruz \#32293); detyrosinated $\alpha$-tubulin (Merck Millipore AB3201); Tyrosinated tubulin (YL1/2 Sigma Aldrich MA1 80017); Flag (CST\#2368); GFP (Santa Cruz \#sc8334). 


\section{Crystallization data collection and processing}

Initial hits of MATCAP were obtained in a sitting drop format using 96-well two-drop MRC plates and the commercial screens PACT, JCSG+, Classics-I \& II, ProComplex, ComPAS, pHClear and Morpheus. The drops consisted of $100 \mathrm{nl}$ protein with $100 \mathrm{nl}$ reservoir solution. After the period of one-week initial hits were identified in the Classics-I screen and further optimized by using Linbro plates. MATCAP diffracting crystals were obtained in $4.3 \mathrm{M} \mathrm{NaCl}$ and 0.1M HEPES- $\mathrm{NaOH}$ pH 7. Crystals appear as clusters, which were further, optimized by using the additive screen from Hampton. Crystals grew to maximum dimensions over a period of two weeks. We were unable to grow single crystals, however by varying the concentration of ethylenediaminetetraacetic acid (EDTA), we succeeded in reducing the number of needles that grew in each cluster, allowing us to harvest a well-shaped three-dimensional tip of a needle that was used for subsequent structure determination. Crystal were harvested and cryo-protected in a solution containing $30 \%$ ethylene glycol and vitrified in liquid nitrogen. A native dataset was collected at beamline PXIII at SLS to $2.1 \AA$ resolution. Diffraction data were integrated and scaled automatically by XDS ${ }^{(18)}$. Mercury derivative crystals were prepared by soaking crystals in methylmercury chloride solution $(10 \mathrm{mM})$ for 3 min prior cyo-protection and vitrification.

\section{Structure solution and refinement}

MATCAP structure was determined using the SIRAS phasing method. The HKL2MAP interface was used to run SHELX program suite using different resolution cutoffs and different number of sites ${ }^{(13)}$. The best solution identified 10 mercury atoms, four with occupancy between 0.88 and 1.0 with the 10th atom having occupancy of 0.39 .

This was considered as a likely solution and then we run the CRANK pipeline with these sites as input ${ }^{(14)}$. CRANK resulted in a model containing 1300 residues fixed in seven fragments; $90 \%$ of the residues were docked in sequence. This model was further used to obtain a molecular replacement solution against the native dataset. The structure was manually completed and refined with several iterations of REFMAC ${ }^{(19)}$ and rebuilding in $\mathrm{COOT}^{(20)}$, including a few rounds of PDB-REDO pipeline ${ }^{(21)}$. The final model has an $R_{\text {free }}$ of $25 \%$, with a clash score of (1.58) and has zero Ramachandran outliers.

\section{Small angle X-ray scattering (SAXS) data collection and analysis}

SAXS data were collected at Beamline B21 at Diamond Light Source (UK). About 40 $\mu$ l of protein sample were loaded onto a Superdex column at a concentration of 3-10 mg ml-1. The scattering profile of the eluate was collected in 600 frames. The protein elution peak was determined from the data using the program CHROMIXS ${ }^{(22)}$ and the scattering intensities of the corresponding frames were averaged. The scattering from the solvent was subtracted using the default parameters. 
The data for different MATCAP truncations were analyzed with both ATSAS and ScÅtter software packages to obtained model independent parameters such as radius of gyration $\left(R_{g}\right)$ maxima distance of the biomolecule $\left(D_{\max }\right)$ and Volume of correlation $\left(V_{c}\right)^{(23,24)}$. The molecular mass was calculated using the Porod, Qr, Bayessian interference and Size and shape method (25-27) Theoretical scattering data for the atomic models of MATCAP were generated using $\mathrm{CRYSOL}^{(28)}$. SREFLEX program from ATSAS suite was used to examine the flexibility of the crystal structure with the experimental SAXS data in solution ${ }^{(29)}$.

Table 1.

\begin{tabular}{|c|c|c|}
\hline & Native & Hg-soak \\
\hline \multicolumn{3}{|l|}{ Data collection } \\
\hline Spacegroup & $P 12_{1} 1$ & $P 12,1$ \\
\hline \multicolumn{3}{|l|}{ Cell dimensions } \\
\hline$a, b, c(\AA)$ & 55.8588 .02165 .61 & 55.73087 .730165 .230 \\
\hline$\alpha, \beta, \gamma\left(^{\circ}\right)$ & 90.090 .6790 .0 & 90.00090 .77990 .000 \\
\hline Wavelength & 0.966 & 1.006 \\
\hline Resolution ( $\AA$ ) & $46.56-2.113(2.189-2.113)$ & $47.04-2.59(10.35-2.59)$ \\
\hline$R_{\text {merge }}$ & $0.07002(0.8073)$ & $0.049(1.75)$ \\
\hline $1 / s(I)$ & $10.18(1.28)$ & $20.7(19.5)$ \\
\hline $\mathrm{CC}_{1 / 2}$ & $0.994(0.69)$ & $0.99(0.72)$ \\
\hline Completeness(\%) & $98.60(97.23)$ & 100(99.7) \\
\hline Redundancy & $3.4(3.3)$ & $20.7(20.4)$ \\
\hline \multicolumn{3}{|l|}{ Refinement } \\
\hline Resolution $(\AA)$ & 2.1 & \\
\hline No. reflections & 90740 & \\
\hline$R_{\text {work }} / R_{\text {free }}$ & $0.2291 / 0.2572$ & \\
\hline \multicolumn{3}{|l|}{ No. atoms } \\
\hline Protein & 10836 & \\
\hline Water & 242 & \\
\hline \multicolumn{3}{|l|}{ B factors } \\
\hline Protein & 21.07 & \\
\hline Water & 44.87 & \\
\hline \multicolumn{3}{|l|}{ R.m.s deviations } \\
\hline Bond lengths ( $\AA$ ) & 0.01 & \\
\hline Bond angles $\left({ }^{\circ}\right)$ & 1.29 & \\
\hline
\end{tabular}




\section{REFERENCES}

1. S. Gadadhar, S. Bodakuntla, K. Natarajan, C. Janke, The tubulin code at a glance. J. Cell. Sci. 130, 1347-1353 (2017).

2. C. Janke, M. M. Magiera, The tubulin code and its role in controlling microtubule properties and functions. Nat. Rev. Mol. Cell Biol. 120, 923-20 (2020).

3. J. Nieuwenhuis et al., Vasohibins encode tubulin detyrosinating activity. Science. 358, 1453-1456 (2017).

4. C. Aillaud et al., Vasohibins/SVBP are tubulin carboxypeptidases (TCPS) that regulate neuron differentiation. Science. 358, 1448-1453 (2017).

5. A. Adamopoulos et al., Crystal structure of the tubulin tyrosine carboxypeptidase complex VASH1-SVBP. Nat. Struct. Mol. Biol. 26, 567-570 (2019).

6. N. Wang et al., Structural basis of tubulin detyrosination by the vasohibin-SVBP enzyme complex. Nat. Struct. Mol. Biol. 26, 571-582 (2019).

7. C. Zhou, L. Yan, W.-H. Zhang, Z. Liu, Structural basis of tubulin detyrosination by VASH2/SVBP heterodimer. Nat Commun. 10, 3212-8 (2019).

8. X. Liu et al., Structural insights into tubulin detyrosination by vasohibins-SVBP complex. Cell Discov. 5, 65-5 (2019).

9. F. Li, Y. Hu, S. Qi, X. Luo, H. Yu, Structural basis of tubulin detyrosination by vasohibins. Nat. Struct. Mol. Biol. 26 583-591 (2019).

10. S. Liao et al., Molecular basis of vasohibins-mediated detyrosination and its impact on spindle function and mitosis. Cell Res. 29, 533-547 (2019).

11. M. P. A. Luna-Vargas et al., Enabling high-throughput ligation-independent cloning and protein expression for the family of ubiquitin specific proteases. Journal of Structural Biology. 175, 113-119 (2011).

12. W. T. M. Mooij, E. Mitsiki, A. Perrakis, ProteinCCD: enabling the design of protein truncation constructs for expression and crystallization experiments. Nucleic Acids Research. 37, W402-W405 (2009).

13. T. Pape, T. R. Schneider, HKL2MAP: a graphical user interface for macromolecular phasing with SHELXprograms. J Appl Crystallogr. 37, 843-844 (2004).

14. P. Skubák, N. S. Pannu, Automatic protein structure solution from weak X-ray data. Nat Commun. 4, 2777-6 (2013).

15. J. Nieuwenhuis, T. R. Brummelkamp, The Tubulin Detyrosination Cycle: Function and Enzymes. Trends Cell Biol. 29, 80-92 (2019).

16. D. Raybin, M. Flavin, An enzyme tyrosylating alpha-tubulin and its role in microtubule assembly. Biochem. Biophys. Res. Commun. 65, 1088-1095 (1975).

17. L. Zimmermann et al., A Completely Reimplemented MPI Bioinformatics Toolkit with a New HHpred Server at its Core. J. Mol. Biol. 430, 2237-2243 (2018).

18. W. Kabsch, XDS. Acta Crystallogr. D Biol. Crystallogr. 66, 125-132 (2010).

19. G. N. Murshudov et al., REFMAC5 for the refinement of macromolecular crystal structures. Acta Crystallogr. D Biol. Crystallogr. 67, 355-367 (2011).

20. P. Emsley, B. Lohkamp, W. G. Scott, K. Cowtan, Features and development of Coot. Acta Crystallogr. D Biol. Crystallogr. 66, 486-501 (2010).

21. R. P. Joosten, F. Long, G. N. Murshudov, A. Perrakis, The PDB_REDO server for macromolecular structure mode optimization. IUCrJ. 1, 213-220 (2014)

22. A. Panjkovich, D. I. Svergun, CHROMIXS: automatic and interactive analysis of chromatography-coupled smallangle X-ray scattering data. Bioinformatics. 34, 1944-1946 (2018).

23. D. Franke et al., ATSAS 2.8: a comprehensive data analysis suite for small-angle scattering from macromolecular solutions. J App/ Crystallogr. 50 (2017), doi:10.1107/S1600576717007786.

24. R. P. Rambo, in Structures of Large RNA Molecules and Their Complexes (Elsevier, 2015), vol. 558 of Methods in Enzymology, pp. 363-390.

25. R. P. Rambo, J. A. Tainer, Characterizing flexible and intrinsically unstructured biological macromolecules by SAS using the Porod-Debye law. Biopolymers. 95, 559-571 (2011).

26. R. P. Rambo, J. A. Tainer, Accurate assessment of mass, models and resolution by small-angle scattering. Nature. 496, 477-481 (2013).

27. N. R. Hajizadeh, D. Franke, C. M. Jeffries, D. I. Svergun, Consensus Bayesian assessment of protein molecular mass from solution X-ray scattering data. Sci Rep. 8, 7204-13 (2018).

28. D. I. Svergun, B. C, K. M. H. J, CRYSOL-a Program to Evaluate X-ray Solution Scattering of Biological Macromolecules from Atomic Coordinates. 28 (1995), pp. 768-773. 
29. A. Panjkovich, D. I. Svergun, Deciphering conformational transitions of proteins by small angle X-ray scattering and normal mode analysis. Phys Chem Chem Phys. 18, 5707-5719 (2016). 



\section{Chapter 5}

General Discussion 


\section{GENERAL DISCUSSION}

Microtubules (MTs) are versatile components of the eukaryotic cytoskeleton, providing a framework that cells can utilize to perform a plethora of functions. It is not surprising that cells have evolved a "tubulin code", that enables MTs specialization ${ }^{(1)}$. MTs are decorated with a number of PTMs that regulate numerous cellular procedures such as mitosis, intracellular transport and migration ${ }^{(1,2)}$. Among PTMs, detyrosination has attracted a lot of attention from the scientific community the last few years, also due a paradox surrounding it: despite being among the first PTMs identified ${ }^{(3)}$, the enzymes that control the whole procedure had remained unknown for more than 40 years ${ }^{(4)}$. (De-)tyrosination controls a number of cellular mechanisms, such as migration, cardiomyocyte contraction, neuronal differentiation, highlighting the importance of the detyrosination cycle, as a tuning mechanism for potential therapies and drug design ${ }^{(1)}$. In this discussion I will focus on the consequences of our experimental work and suggest new questions that need to be addressed to further understand the importance of the tubulin code.

\section{The discovery of two novel enzymes with detyrosinating activity}

Our collaborators used a powerful genetic approach which combines haploid genetics with cell sorting, to identify regulators of detyrosination ${ }^{(4)}$. The discovery of SVBP as a positive regulator of detyrosination (4) shed light to the 40-year-old enigma, providing the basis to understand the role of detyrosination in molecular detail. As SVBP was known to be a binding partner of the vasohibin family, that led to the identification of the complex of vasohibins with SVBP as the elusive enzyme that detyrosinates $\alpha$-tubulin ${ }^{(5)}$. In vitro experiments with recombinant proteins showed that MTs are the preferred substrate, despite VASH1-2/SVBP being able to detyrosinate free $\alpha / \beta$-tubulin heterodimers ${ }^{(4)}$. One of the most intriguing findings that came up during the discovery of vasohibins was that upon depletion of vasohibins, cells still contain a residual detyrosination activity towards $\alpha$-tubulin ${ }^{(4)}$. This highlighted the complexity of the tubulin code, and indicated that additional enzymes could potentially detyrosinate $\alpha$-tubulin. To identify the additional regulators of detyrosination, our collaborators used the same well-established genetic methodology that was used to identify vasohibins, but this time on a vasohibin-deficient background. The results of this study identify MATCAP (Microtubule Carboxypeptidase Protein) as a potential regulator of detyrosination.

It is of interest that both detyrosinating enzymes were not classified as carboxypeptidases earlier, highlighting that their function could not easily be predicted by their sequence. Our work, presented in this thesis, is the first step towards the structural and functional characterization of both regulators of detyrosination. In particular, the structural characterisation of MATCAP (part of a more complete ongoing collaborative research effort) not only adds a new player in the enzymatic repertoire of the tubulin code, but also creates a number of biological questions, indicating the complexity of the tubulin code and its constituents (see box-1). 
Box 1. Outstanding questions

1. Why do cells have multiple detyrosinating enzymes?

2. What is the specificity of MATCAP- does it act on MTs or heterodimers?

3. Do MATCAP and VASH1/2-SVBP show different specificities on MTs?

4. Is there a mechanism that regulates MATCAP-detyrosination of MTs versus vasohibin-detyrosination?

5. Could other PTMs or binding partners coordinate a specific enzyme-driven detyrosination?

Until now the main substrate known for vasohibins, remains the C-terminal tails of MTs. Our results show that vasohibins can remove the last aromatic residue (tyrosine or phenylalanine) from a highly negatively charged tail where the penultimate residue is a glutamate ${ }^{(6)}$. The C-terminal tails of $\alpha$-tubulin show a high degree of conservation; however, it is still unknown if there are other proteins that are physiologically relevant substrates of VASH1/2-SVBP. It is of great interest to understand the interplay between MATCAP and vasohibins. What defines the specificity towards their substrate for both MATCAP and vasohibins? It is still unknown if MATCAP can detyrosinate other proteins or if it acts in a synergistic way with vasohibins.

\section{Implications of the crystal structures of the VASH1/SVBP and MATCAP tubulin tyrosine carboxypeptidases}

Experiments in cells and in vitro assays, showed that the presence of SVBP increases the solubility of vasohibins and as a consequence this has an effect on the activity of the complex. To further understand the structural interplay between the two components of the detyrosinating complex, we solved the crystal structure of VASH1/SVBP. A main feature of the VASH/SVBP structure was that SVBP adopts a helical conformation, which acts as a molecular chaperone, stabilizing the structural core of VASH1. Both N-/C-termini of VASH1/SVBP are less well-conserved compared to the main structural core and this is also highlighted by the lack structural information for these disordered regions in all structures deposited in the PDB ${ }^{(6-10)}$. SEC-SAXS experiments with the fulllength complex, revealed the dynamic nature of both termini. One possible role for these highly disordered and flexible regions is through protein-protein interactions with other biomolecules and/or MTs. Upon interaction with a binding partner, or a substrate (e.g MTs) these regions could become structured creating a binding interface.

To understand the mode of tubulin tail binding to VASH1/SVBP, we performed molecular docking experiments using HADDOCK ${ }^{(11)}$; the resulting models were experimentally validated with in vivo experiments in cells. Based on our docking model and biochemical experiments we confirmed residues Cys-169, His204 and Leu-226 to consist of the catalytic triad of VASH1/SVBP. In addition, at the same time three independent groups solved the structure of VASH1/SVBP or VASH2/SVBP in complex with inhibitors or $\alpha$-tubulin mimicking peptides, further confirming our suggested model $(7,8,10)$. Our results confirmed the role of VASH1/SVBP as a tubulin carboxypeptidase, also providing a framework for design of inhibitors for further study of carboxypeptidases.

The structural characterization of MATCAP, showed that the catalytic activity resides in a 
C-terminal domain, whereas the N-terminal region is disordered. Biochemical data showed the metal-dependent nature of MATCAP for its activity, and the structure confirmed that MATCAP requires $\mathrm{Zn}^{2+}$ ions to detyrosinate tubulin heterodimers. The metal-dependent nature of MATCAP is also one of the main differences compared to vasohibins, indicating an activation mechanism upon presence of a metal ion. Details of the binding mode and specificity are being elucidated at the present time, with the help of the structure that I determined and presented earlier as an experimental chapter in this thesis. While many questions about specificity and substrate binding remain, a most interesting one is the role of the N-terminal disordered region. This is a recurring characteristic, also observed in the VASH1-SVBP complex, where disordered/flexible regions surround a highly structured compact fold. We can easily hypothesize that disordered regions could participate in protein-protein interactions with other regulators or microtubules. The MATCAP structure also contains some degree of flexibility in the catalytic domain.

MATCAP appears to be a dynamic macromolecule that binds onto MTs, detyrosinates them and as a result functionally specializes them. We speculate that the nature of MATCAP should be adaptive to the microenviroment of MTs. MTs are decorated with a plethora of PTMs, such as polyglutamylation and polyglycylation among others, which create a microenvironment where MATCAP should recognize the target region, bind onto it, and detyrosinate it ${ }^{(12)}$. So far, no data exists on what drives a MATCAP-dependent detyrosinating activity of MTs. Does MATCAP recognizes the C-terminal tail of tubulin only, or binding to the MTs triggers detyrosination? What is the effect of other PTMs on the regulation of MATCAP? Does MATCAP act as a carboxypeptidase for the C-terminal tyrosine or other residues can be equally processed? We know that vasohibins act specifically as carboxypeptidases of aromatic residues (Tyrosine and Phenylalanine can also be well-processed) (4) does MATCAP show a similar specificity towards aromatic residues as well? Of equal interest is the mode of binding and recognition of microtubules. To address this question effort should be made on resolving the structure of the complex of both proteins bound onto microtubules.

So far binding partners for MATCAP and vasohibins, other than SVBP for vasohibins, have not been identified, and this creates a number of scientific questions. What is the role of the disordered $\mathrm{N}$-/C- termini on substrate binding and recognition? Could these disordered regions interact with MTs or act as scaffolds for interactions with other proteins? How do other post-translational modifications affect a vasohibin-SVBP detyrosination of alpha-tubulin compared to a MATCAP-dependent detyrosination?

\section{Opportunities from the structural work on detyrosinating enzymes}

Structural biology can provide the biological community with atomic detail information for al the enzymatic steps of the tyrosination/detyrosination cycle. X-ray crystallography has been the workhorse for more than 50 years helping us to understand the structure/function of 
proteins. Nowadays a continuous effort has been made towards improvements of instruments and techniques in the cryo-EM field, which enable us to study macromolecular complexes close to atomic resolution overcoming the bottleneck of protein crystallization. Purification protocols for tubulin and assembly of MTs with homogeneous purified tubulin have now been made possible, consisting a valuable tool for studying MT-bound proteins.

We now, live during "the resolution-revolution" era where cryo-EM has become an indispensable technique in structural biology. The cryo-EM community is rapidly growing; new software developments allow the helical reconstruction of microtubule-bound proteins, providing structural details at near-atomic resolution ${ }^{(13)}$. Protocols for helical reconstruction of MT-bound proteins have been established and are now routinely used by many labs ${ }^{(13)}$. Technological advancements in tubulin purification and assembly of MTs with homogeneous PTMs will give a better understanding on the role of detyrosination in cells, and how this affects MT assembly and dynamics. Therefor a cryo-EM reconstruction of the complex between VASH1-SVBP and or MATCAP and homogeneously purified and decorated, with a certain PTM, microtubules will reveal the interplay between detyrosination and other PTMs. Finally, our work presented in this thesis provides the basic structural information, for further design of small molecule as inhibitors or regulators for both detyrosinating enzymes.

A complex between MATCAP and the tubulin heterodimer will give us insight on how the C-terminal tail is recognized and processed. Of equal importance is to understand if there is a synergistic interplay among post-translational modifications. MTs contain a plethora of PTMs that increases the complexity and the encoded information they contain, and should not be studied only on the level, of each PTM individually. Combinations of PTMs could encode for different functional specializations and promote interactions with proteins/effectors that have to be identified. Structure determination of complexes, between homogeneously purified and decorated microtubules with certain PTMs and the respective enzymatic regulators, can shed light on the complexity of the tubulin code ${ }^{(14)}$.

The discovery and structure determination of MATCAP (presented in chapter-4) together with the VASH1-SVBP structure (presented in chapter-3) acts as a first step towards a complete overview of the enzymatic regulators of the tubulin code. Over the next years structural characterisation of the complex between MATCAP and MTs will shed light on the molecular mechanism of detyrosination. Both crystal structures provide a scaffold for drug designing of small molecules and inhibitors that regulates detyrosination, and could have pharmaceutical potential in diseases that are connected with deregulation of detyrosination, as in e.g. cardiomyocyte contraction and neuronal development. 


\section{References}

1. J. Nieuwenhuis, T. R. Brummelkamp, The Tubulin Detyrosination Cycle: Function and Enzymes. Trends Cell Biol. 29, 80-92 (2019).

2. P. Strzyz, Post-translational modifications: Extension of the tubulin code. Nat. Rev. Mol. Cell Biol. 17, 609 (2016).

3. D. Raybin, M. Flavin, Enzyme which specifically adds tyrosine to the alpha chain of tubulin. Biochemistry. 16 2189-2194 (1977).

4. J. Nieuwenhuis et al., Vasohibins encode tubulin detyrosinating activity. Science. 358, 1453-1456 (2017).

5. Y. Suzuki et al., Isolation of a small vasohibin-binding protein (SVBP) and its role in vasohibin secretion. J. Cell. Sci. 123, 3094-3101 (2010).

6. A. Adamopoulos et al., Crystal structure of the tubulin tyrosine carboxypeptidase complex VASH1-SVBP. Nat. Struct. Mol. Biol. 26, 567-570 (2019).

7. N. Wang et al., Structural basis of tubulin detyrosination by the vasohibin-SVBP enzyme complex. Nat. Struct. Mol. Biol. 26, 571-582 (2019)

8. X. Liu et al., Structural insights into tubulin detyrosination by vasohibins-SVBP complex. Cell Discov. 5, 65-5 (2019).

9. C. Zhou, L. Yan, W.-H. Zhang, Z. Liu, Structural basis of tubulin detyrosination by VASH2/SVBP heterodimer. Nat Commun. 10, 3212-8 (2019).

10. S. Liao et al., Molecular basis of vasohibins-mediated detyrosination and its impact on spindle function and mitosis. Cell Res. 29, 533-547 (2019).

11. C. Dominguez, R. Boelens, A. M. J. J. Bonvin, HADDOCK: a protein-protein docking approach based on biochemical or biophysical information. J. Am. Chem. Soc. 125, 1731-1737 (2003).

12. C. Janke, M. M. Magiera, The tubulin code and its role in controlling microtubule properties and functions. Nat. Rev. Mol. Cell Biol. 120, 923-20 (2020)

13. A. D. Cook, S. W. Manka, S. Wang, C. A. Moores, J. Atherton, A microtubule RELION-based pipeline for cryo-EM image processing. Journal of Structural Biology. 209, 107402 (2020).

14. A. Vemu, C. P. Garnham, D.-Y. Lee, A. Roll-Mecak, Generation of differentially modified microtubules using in vitro enzymatic approaches. Meth. Enzymol. 540, 149-166 (2014). 



\section{Addendum}

Summary

Nederlandse samenvatting

Curriculum Vitae

List of Publications

Acknowledgements 


\section{SUMMARY}

Microtubules are essential components of the eukaryotic cytoskeleton. They are subjected to a number of post-translational modifications, which increase their heterogeneity, leading to functional specialization. The combination of the post-translational modifications, together with the differential overexpression of tubulin isotypes, creates the tubulin code. This thesis focuses on detyrosination, one of the post-translational modifications that occur on the C-terminal tail of $\alpha$-tubulin. Using a combination of structural biology tools, including Small angle X-ray scattering and X-ray crystallography, and biochemical and biophysical assays, I present the structure and the mechanism of the two novel tubulin detyrosinating enzymes, which remained elusive for four decades, and were discovered by our collaborators.

In chapter 2, we describe how Nieuwenhuis et al used a powerful approach based on haploid genetics to identify regulators of tubulin detyrosination. Briefly, antibodies that specifically recognize the two differentially tyrosinated forms of $\alpha$-tubulin, were used to stain mutagenized HAP1 cells pretreated with paclitaxel, a microtubule-stabilizing agent. Mutagenised cells were then subjected to fluorescence-activated cell shorting (FACS) to separate the two populations exhibiting high and low levels of detyrosination. A search for genes enriched in mutations in the two distinct cell populations that differ in the detyrosination levels, revealed the known Tubulin Tyrosine Ligase TTL as a negative regulator of detyrosination, and resulted in the discovery of SVBP as a positive regulator. SVBP is the binding partner of vasohibins (VASH1, $\mathrm{VASH} 2$ ), a family of protein that was thought to play a role in angiogenesis. In humans, both paralogues VASH1 and VASH2 form a complex with SVBP; which we showed that it has has detyrosinating activity. The VASH1/2 complex with SVBP, can detyrosinate both $\alpha / \beta$-tubulin heterodimers and MTs, showing a preference on their natural substrate, MTs.

In chapter 3, I show the crystal structure of the VASH1/SVBP complex, to further characterize the role of SVBP in the complex and understand how does VASH1 recognizes and binds to the $C$-terminal tail of $\alpha$-tubulin. The adaptation of a helical conformation for SVBP, penetrating the structural core of VASH1 was a striking finding. Our crystal structure shows in atomic detail how SVBP stabilizes VASH1, acting as a molecular chaperone. Using SAXS, we characterized the disordered N/C-termini that VASH1/SVBP complex has, and found that they are highly dynamic and behave as intrinsically disordered regions. Using the molecular modeling HADDOCK algorithm we proposed a model for the binding and recognition of the $\mathrm{C}$-terminal tyrosine of $\alpha$-tubulin, which was experimentally validated with mutagenesis studies in cells.

The identification of the VASH/SVBP activity marked the beginning of an end in the 40-year old enigma in the tubulin code field. A remaining detyrosinating activity was detectable in cells that lack both vasohibins. This led to the hypothesis that cells contain a vasohibin-independent 
way to detyrosinate $\alpha$-tubulin, possibly by the existence of additional detyrosinating enzymes.

In chapter 4, I build on the identification of MATCAP by Lisa Landskron, as the last elusive detyrosinating enzyme in humans. To understand in molecular detail the role of this new detyrosinating enzyme, I present the structural characterisation of MATCAP. Using SAXS, I characterized the domain organization of MATCAP and identified the catalytic domain (MATCAP-CD). I then describe the determination of the crystal structure of MATCAP-CD, which classifies it as a metal-dependent carboxypeptidase, requiring a divalent cation $\left(\mathrm{Zn}^{2}\right)$ for its activity. The MATCAP structure was the trigger for many follow-up experiments on the characterization of specific residues and substrates that are still ongoing.

I conclude with a general discussion in chapter $\mathbf{5}$ where I present our findings in a broader context. 


\section{NEDERLANDSE SAMENVATTING}

Microtubuli zijn essentiële componenten van het eukaryote cytoskelet. Ze ondergaan verschillende posttranslationele modificaties. Dit versterkt de heterogeniteit, wat leidt tot een meer gespecialiseerde functie. De combinatie van posttranslationele modificaties, samen met de verschillende overexpressies van isotypes van tubuline, vormt de tubuline code. Dit proefschrift focust op detyronisatie, dit is een van de post-translationele modificaties die kunnen plaatsvinden op de carboxyterminale staart van $\alpha$-tubuline. We hebben gebruik gemaakt van structuur biologische technieken zoals kleine hoek röntgen verstrooiing (SAXS) en röntgenkristallografie, en biochemische en biofysische methoden. Hier, laat ik de structuur en het mechanisme zien van twee nieuwe tubuline detyronisatie enzymen, die de afgelopen 40 jaar nog niet ontdekt waren en nu ontdekt zijn door onze collaborators.

In hoofdstuk 2 bespreken we hoe Niewenhuis et al. een methode gebruiken die gebaseerd is op haploïde genetica, en hoe de nieuwe regulators van tubuline detyronisatie zijn geïdentificeerd door deze methode toe te passen. Antilichamen die twee gedifferentieerde tyrosinatie versies van $\alpha$-tubuline herkennen, zijn gebruikt om gemuteerde HAP1 cellen te kleuren met paclitaxel, een middel dat microtubuli stabiliseert. De gemuteerde cellen werden gesorteerd met behulp van flow cytometrie. De cellen werden verdeeld in twee groepen cellen; met hoge detyronisatie niveaus en met lage detyronisatie niveaus. In beide groepen werd gezocht naar verschillen in intracellulaire fenotypes, waaronder een verschil in detyronisatie. Hierdoor werd Tubiline Tyrosine Ligase (TTL) ontdekt als een negatieve regulator van detyronisatie, en SVBP werd ontdekt als positieve regulator. SVBP gaat interacties aan met vasohibins (VASH1, VASH2), dit is een eiwitfamilie die mogelijk een rol speelt bij angiogenese. In mensen, zowel VASH1 en VASH2 vormen een complex met SVBP en dit complex heeft detyrosinatie activiteit. Het VASH1/2 complex met SVBP kan zowel $\alpha / \beta$-tubiline detyroniseren als microtubuli, maar met een voorkeur voor hun natuurlijke substraat, de microtubuli.

In hoofdstuk $\mathbf{3}$ laat ik de kristalstructuur van VASH1/SVBP-complex zien. Hiermee kunnen we de rol van SVBP in het complex beter karakteriseren en begrijpen hoe VASH1 de carboxyterminale staart van $\alpha$-tubuline herkent en bindt. Het was een onverwachte ontdekking dat SVBP een $\alpha$-helix conformatie aannam, waardoor het kon doordringen in de kern van VASH1. Onze kristalstructuur laat in atomische details zien hoe SVBP, VASH1 stabiliseert als moleculaire chaperonne. Met gebruik van de SAXS-techniek, hebben we de ongeordende N/C-eindes van de VASH1/SVBP complex gekarakteriseerd en ontdekten we dat ze zeer dynamisch zijn en zich gedragen als intrinsiek verstoorde regio's. Met gebruik van een moleculaire modeleer algoritme, HADDOCK, hebben we een model voorgesteld voor de herkenning en de binding van de carboxyterminale tyrosine van $\alpha$-tubuline met VASH1/SVBP, en dit is experimentee gevalideerd met mutagenese experimenten in cellen. 
De ontdekking van de VASH/SVBP activiteit resulteerde in het begin van het einde van een 40-jaar oud raadsel in de wereld van tubuline codes. Maar toch was er nog resterende detyronisatie activiteit detecteerbaar in cellen waarbij de vasohibins afwezig waren. Dit leidt tot de hypothese dat cellen ook een vasohibin-onafhankelijke manier hebben om $\alpha$-tubuline te detyroniseren. Mogelijk door de aanwezigheid van extra detyronisatie enzymen.

In hoofdstuk 4, heb ik verder gewerkt aan de ontdekking van MATCAP (door Lisa Landskron), de laatste onvindbare detyronisatie enzym in mensen. Om de rol van dit nieuwe detyronisatie enzym beter te begrijpen op moleculair niveau, laat ik de structurele karakterisering van MATCAP zien. Met gebruik van SAXS, heb ik de domein organisatie van MATCAP gekarakteriseerd en het katalytische domain gevonden (MATCAP-KD). Daarna beschrijf ik de bevinding van de kristalstructuur van MATCAP-KD, dit eiwit is een metaal-afhankelijke carboxypeptidase, die een divalent cation $\left(\mathrm{Zn}^{2+}\right)$ nodig heeft voor zijn activiteit. De MATCAP-structuur was de trigger voor vele opvolgende experimenten voor het karakteriseren van de specifieke residuen en substraten en dit is duurt nog steeds voort.

Ik sluit af met een algemene discussie in hoofdstuk 5, waar ik onze resultaten laat zien in een bredere context. 


\section{CURRICULUM VITAE}

Athanassios (Nassos) Adamopoulos was born on the 17 $17^{\text {th }}$ of February 1987 in Athens, Greece. He graduated form the Department of Molecular Biology and Genetics in Alaxandroupoli, University of Thrace, Greece. After spending three years working and studying in the UK he graduated with MRes in Cell and Molecular biology from the University of Edinburgh. He spent one year in Greece for the obligatory military service, before leaving for the Netherlands, to work in the group of Anastassis Perrakis this time as a PhD student. During this period he spent one year as a visiting student at University of Southern California, in the lab of prof. Raymond Stevens, where he worked in the field of G-protein coupled receptors. He returned to Amsterdam to continue his research on membrane proteins and a number of other projects including JBP1 and tubulin modifying enzymes. 


\section{LIST OF PUBLICATIONS}

Martinelli,L., Adamopoulos,A., Johansson,P., Wan,P.T., Gunnarsson,J., Guo,H., Boyd,H., Zelcer,N. and Sixma,T.K. (2020) Structural analysis of the LDLR-interacting FERM domain in the E3 ligase IDOL. J. Biol. Chem., 295, 13570-13582.

Adamopoulos,A*., Heidebrecht,T*., Roosendaal,J., Touw,W.G., Phan,I.Q., Beijnen,J. and Perrakis,A. (2019) The domain architecture of the protozoan protein J-DNA-binding protein 1 suggests synergy between base J DNA binding and thymidine hydroxylase activity. J. Biol. Chem., 294, 12815-12825. *shared first author

Adamopoulos,A*., Landskron,L*., Heidebrecht,T., Tsakou,F., Bleijerveld, O.B., Altelaar,M., Nieuwenhuis,J., Celie,P.H.N., Brummelkamp,T.R. and Perrakis,A. (2019) Crystal structure of the tubulin tyrosine carboxypeptidase complex VASH1-SVBP. Nat. Struct. Mol. Biol., 26, 567570. *shared first author

van Beusekom, B., Heidebrecht,T., Adamopoulos,A., Fish,A., Pardon, E., Steyaert,J., Joosten, R.P. and Perrakis,A. (2018) Characterization and structure determination of a llama- derived nanobody targeting the J-base binding protein 1. Acta Crystallogr F Struct Biol Commun, 74, 690-695.

Nieuwenhuis,J., Adamopoulos,A., Bleijerveld,O.B., Mazouzi,A., SRckel,E., Celie,P., Altelaar,M., Knipscheer,P., Perrakis,A., Blomen,V.A., et al. (2017) Vasohibins encode tubulin detyrosinaRng activity. Science, 358, 1453-1456.

Hiruma,Y., Sacristan,C., Pachis,S.T., Adamopoulos,A., Kuijt,T., Ubbink,M., Castelmur,von,E., Perrakis,A. and Kops,G.J.P.L. (2015) Competition between MPS1 and microtubules at kinetochores regulates spindle checkpoint signaling. Science, 348, 1264-1267. 


\section{ACKNOWLEDGEMENTS}

A PhD is a journey towards knowledge, with some intermediate stops including scientific achievements and publications. Although many people believe that obtaining the title is the main target, I would say that the journey itself is the true meaning of a PhD. The failed experiments, the difficult projects the experiences that someone collects during these years will shape the scientific character of the candidate and definitely not the title. Of course, this journey could not have been possible without the help and interaction with a number of people that I would like to personally acknowledge.

First of all I would like to thank my supervisor Anastassis Perrakis, who gave me this great opportunity to work in his lab. He gave me the freedom (or challenges) to conduct my own experiments and trying a number of projects some of them successful some of them more "challenging". The knowledge and the skills I acquired will be a valuable tool for my future career therefore I am grateful for that. I will definitely miss the fact that we both agree to disagree in almost everything, a characteristic that gave rise to fruitful discussions, for almost all aspects of life including experiments, science and politics.

I would also like to thank my copromotor, Thijn for his constructive comments during work discussions and constant interest on my experiments that have been the inspiration for me, to study this extremely interesting protein targets presented in this thesis.

I would also like to thank you, Titia, for your critical and constructive comments during presentations and committee reports.

My PhD supervisory committee consisting of prof. Titia Sixma, prof. Anastassis Perrakis, prof. Thijn Brummelkamp, prof. Hein te Riele and prof. Kees Jalink. I would like to thank you all, for your support and critical comments during my studies. My thesis committee consists of prof. Anna Akhmanova, prof. Marc Baldus, prof. Alexandre Bonvin prof. Piet Gros, prof. Titia Sixma thank you for making this day possible.

Tati, there are no words to express my gratitude to you. You were always there with a smile helping me overcoming all the scientific and personal obstacles that could appear. Our legendary "acetic team" managed to handle all the impossible projects we were given and continued doing experiments finishing and publishing our results despite all the voices that were saying the opposite. I will never forget the fun we had inside and outside the lab, and I will be definitely coming to Amsterdam to continue our traditions with the other important member of the team ;) 
Willem-Jan, it was a pleasure working with you and great fun, I will never forget all the advises for both my personal life and scientific career. I will try to keep them and apply them all I promise. Your hard-working skills, and your way to make everything look so simple and feasible was a driving force for me, to study the almost impossible projects we were given. By the way I hope now that you read this, you are done with painting in your house as well.

Pim, your presence in the lab it was a guarantee for all PhD students, that every scientific problem has an answer and you know it. Our discussions and coffees with the other members of the last-office-in-the-corridor were definitely the funniest and most entertaining parts of the day. I will always be grateful to the tips/tricks of protein purification you were providing all off us and looking forward to see the pictures from your biking trip to Santiago.

Shreya, (the Boss) sharing the office with you was an experience by itself. I will never forget our long shifts in the lab listening to some amazing music (West Coast hoods) and of course the great Indian food that you were always bringing. I truly enjoyed our coffee sessions and all 16:00 o' clock experiments, with the other usual suspect of the scientific gang. I would also like to thank your wife Mayuri, for the invitations to your place and my introduction to the delicious Indian cuisine. I wish you all the best for your future career and never forget, Shreya-Leads ;)

Luciiii, (don Martino) what can I say, your energy, positive attitude, coffee-drinking habits, and hard working skills were the engine of our office. I had great fun collaborating with you on IDOL project and see how awesome you are, working both at the bench and with computers, simultaneously, like a tornado. I am sure you are going to achieve great things at J\&J and will definitely keep in touch for all the crazy ideas we have together with the Boss

Doreth, we began the journey of our PhDs together, started by sharing the same office, finishing by sharing the same office. I will always admire your Dutch directness, managing and planning skills. Your accomplishments in life and science are a real example of your strong personality. I wish all the best for you and your amazing family. (Lela, my dear niece, thank you very much for your smiles, and making my life more beautiful every time I see your face)

Bart, what my experience in Amsterdam would be, without you being my flatmate. Your calm way of dealing with everything in life, is a great example for a Mediterranean like me, I am sure you enjoy your life with Rene and hope to see you for holidays in Greece very soon. (The van Beusekom holiday rule, is for sure something I will try to keep and apply)

Mishba, you are a great colleague and a great friend. I am thankful for all the nice moments we had in the lab, constantly teasing Shreya and the help you were always providing me regarding data processing questions. I will always remember our walks and discussions during 
the coronavirus lockdown. I wish you all the best for your future career, for both you and your family.

Elisa, thanks for being a great office-neighbor, and the best cell-biology teacher despite the difficulty of the task and the outcome of the experiment. The Mediterranean air you brought in the office together with the great discussions regarding food, Spain, and the house in Malaga are great examples of the great time we all had. I hope both you and Juanma move to Spain, and I am looking forward for the big party you will give at your house in Malaga.

Foteini, you were the first student that in theory I supervised. Your positive energy was highly appreciated and the help you gave me for the VASH1 project. I hope you enjoy your time in Denmark and wish you a successful career in science. (I will always remember to leave for some time (days) the protein in the fridge, particularly for difficult-to-crystallize samples).

Patrick, you are one of best colleagues someone can ask to work with. Always there, happy and motivated to answer all my questions regarding instruments, experiments, projects. I really enjoyed our collaboration in the lab on the vasohibins project, and definitely will keep in touch for future and further discussions/collaborations.

Magda \& Justina, many thanks for your help, every time I needed something from the Protein Production Facility.

John, thank you very much for the great quality insect cells you are always providing. Without your great work a lot of the experiments described in this thesis would not be possible.

Yvette, you are a great lab manager and the best lab-bench neighbor. The fact that you do all the lab managing work, and in the same time conduct your research is a great example of the kind of scientist you are. Thank you for all your help with the GDE project.

Sasha, I cannot imagine what the lab would be without you. Your deep knowledge in science and biophysics will never be forgotten. Thank you for your help with all biophysical experiments and the great discussions we had regarding history, politics and the army.

Xiaohu, thank you for all the help and the discussions we had in the lab. I am sure you will get your cryo-em structure very soon and looking forward to see it.

Maria, you joined the lab when I was almost finishing, so I wanted to wish you good luck with the biophysical/cell biology experiments for these amazing enzymes. I am sure Lisa will have a great collaborator for the project and all the best for your future career. 
Andrea thank you so much for your advises regarding all computational problems. Your deep and detailed knowledge on things is an asset for the people in the lab. I will never forget our discussions regarding history, Mediterranean culture and politics. And yes if something does not work is definitely my fault!

Robert, thank you for being an amazing colleague, I will never forget the three most important ingredients for a successful scientific experiment. Toasties in the office have to be well-done, eat salty drop in between experiments, stay healthy by leaving the lab eating an apple. I wil definitely miss the great time we all had the office and in the dark room. I hope all the best for you and your protein production facility in Leiden, and definitely try to collaborate with you in the future, for some great science, beers, and what else... Toasties.

Yoshi, I was your student when I first joined the lab. I will always be grateful for your time introducing me into the lab techniques and the great fun we had working on the Mps1 project. Your are definitely the most hard-working person I have ever seen and a great teacher. I wish you all the best for your future for both you and your family.

Robbie, thank you for all the help with protein structure refinement. It was really fun going out for drinks and discuss about life, science and politics. I am looking forward for the new pipeline you will definitely develop, this time for EM-structures, and my suggestion to that would be: Reconstructions, Gotta REDO-EM all !!!

Serge, the latest addition in our amazing office (the notoriously known as Officina de frustrasion), thank you very much for the great discussions we had and the fun trying to create this Wall of Fame with SD quotes. I am sure you will continue the great tradition of Pim and I wish you good luck for all your projects. (We should definitely try this gyros place in Utrecht, sooner or later).

Farid, or Mr Chemist, thanks first of all for the great interaction during lunches and coffee breaks, our brainstorming sessions regarding life and experiments have been engraved in memory. Also, I should thank you for all the reagents you kindly provide all members of the department.

I would also like to thank the other people from the Department of Biochemistry: Ivette, Roy, Susanne, Niels, Shun-Hsiao, Anu, Jitske, Krista, Ida, Hans, Danielle, Nicolaas, Peter, Lisa, GianLuca, and prof. Moolenaar; and former members, Danny, Flora, Wouter, Thanga, since you have all contributed to make the department an ideal environment to work. I wish you all good luck with your future projects and happy cryo-EMing.

Success in science means collaborations; therefore, I would like to specially acknowledge my 
collaborators from the other side of the floor.

Joppe, thank you very much, for dragging me into this amazing field of tubulin modifying enzymes. I will really miss your scientific way of thinking. I believe that we indeed manage to start the "revolution" as we were discussing. It was great fun working with you and I wish you all the best for your future as well.

Lisa Landskron, thank you very much for the great collaboration we had. I had so much fun working together, combining a structural and protein-oriented point of view with you amazing skills in genetics. I am sure you will do great continuing working on tubulin modifying enzymes, and I will definitely keep an eye on the great things you will achieve in the near future.

Jacqueline, thank you for the great discussions we had and your amazing black sense of humor. I am sure you will follow your heart and do what really makes you happy in the future, and guess; success in guaranteed.

Abdel, you are a real scientist, hardworking, full of crazy ideas, that you are not afraid of trying them, and create your own path towards the top. I do not know what your next step in your career will be, but I am definitely sure it will be a successful breakthrough. Keep up the good work, and I will for sure keep an eye for the amazing work you are doing.

Astrid, thanks for the great questions you always had regarding my projects they were very inspiring and to the point. I wish you good luck for your projects as well and I am sure your great discovery is there waiting for you to pick it up.

Mirna, many thanks to you; for all the paperwork and bureaucracy that you can deal so effectively for all member of B8. I wish all the best for your future.

The NKI has been a great environment to work and also gave me the opportunity to meet some really valuable people in my life.

Angela (dotoressa Collarino), my great friend, I will never forget our amazing experiences we had in Amsterdam as flatmates in Louweshoek. I really miss the amazing trip in Roccanova with you and Antonio. I wish all the best for you and Antonio and soon I will plan to visit you in Italy (Castroni, Venchi, Pompi) Greetings to mama-Giusepina, papa-Pasquale.

Apostolos and George, I will never forget our funny chats and coffee breaks we had. They were the ideal recharging sessions after a long day in the lab. I wish good luck to both of you guys for your life and career. 
Alena, thank you for showing me how a real scientist should be, dedicated, determined, data driven, with analytical way of thinking. I am sure you will do great in science and soon you will develop your own group. All the best.

Fariborz, thank you for the great time we had as flatmates in US. Your knowledge is an unlimited source of tricks to tackle every scientific problem; I will never forget the amazing Persian food and tea sessions we had, while discussing life, science, and challenging experiments.

Finally, I would like to thank some close friends that all these years of my studies have always stayed by my side supporting me.

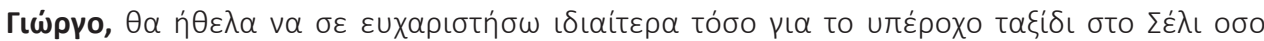

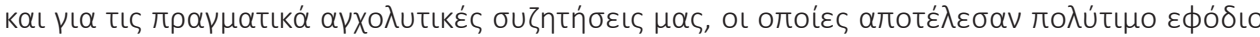

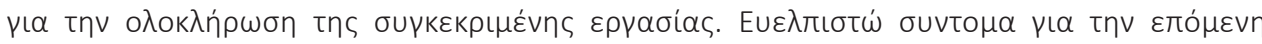

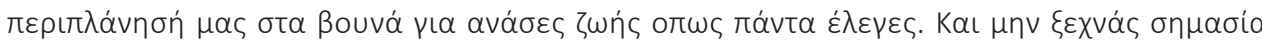

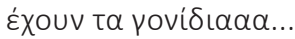

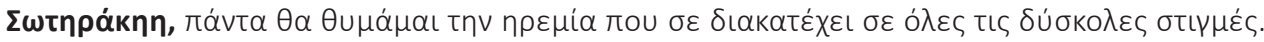

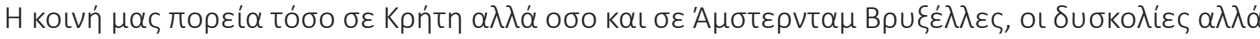

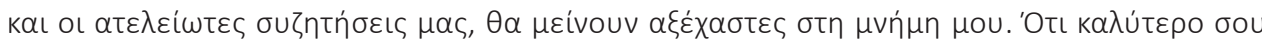

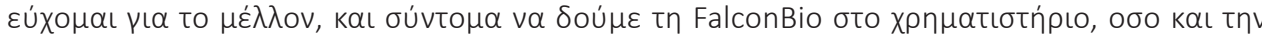

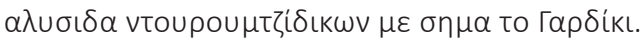

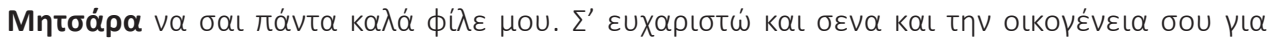

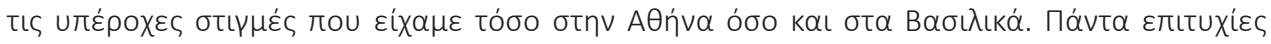

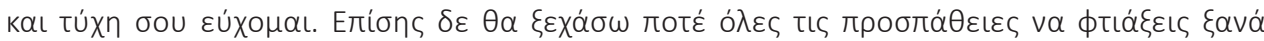

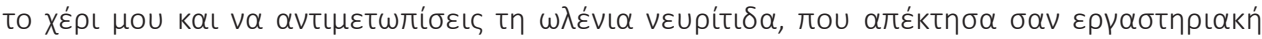

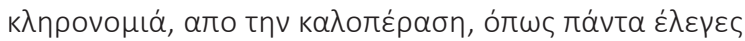

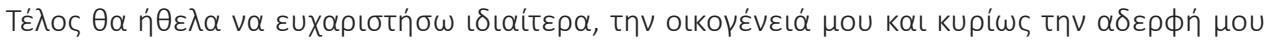

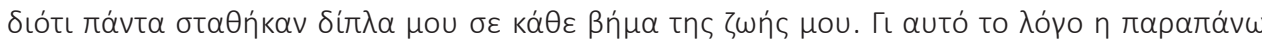

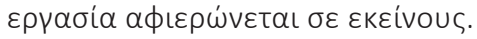


